\title{
Energy Levels and Classified Lines in the First Spectrum of Technetium (Tcl)
}

\author{
W. R. Bozman, C. H. Corliss, and J. L. Tech \\ Institute for Basic Standards, National Bureau of Standards, Washington, D.C. 20234
}

(August 6, 1968)

\begin{abstract}
Progress in the classification of Tc I lines is reported. About 2200 of the known Tc I lines between 2154 and $8918 \AA$ are now classified as transitions between 108 even and 147 odd energy levels. Tables of levels and classified lines are given.
\end{abstract}

Key Words: Atomic spectra; energy levels, Te I; spectrum of technetium; technetium.

\section{Introduction}

The analysis of the first spectrum of technetium has progressed in three separate stages. The first useful description of the spectrum was made by Meggers and Scribner [1]. ${ }^{1}$ The initial advances in the analysis, made by Meggers [2] from that line list, resulted in the classification of 200 lines of Te I as combinations between 19 even and 44 odd energy levels, arising from 5 even and 2 odd configurations. A striking result of Meggers' analysis was that the lowest level of the $4 d^{6} 5$ s configuration, a ${ }^{6} \mathrm{D}_{4 \frac{1}{2}}$, occurs only $2573 \mathrm{~cm}^{-1}$ above the $4 d^{55 s^{2}{ }^{6} \mathrm{~S}}$ ground level. This may be compared with the value $17052 \mathrm{~cm}^{-1}$ for the lowest $3 d^{6} 4 s$ level in $\mathrm{Mn}$ I relative to the $3 d^{5} 4 s^{2}{ }^{6} \mathrm{~S}$ ground level, and with the value 11754 $\mathrm{cm}^{-1}$ for the position of the lowest $5 d^{6} 6 s$ level above $5 d^{5} 6 s^{2}{ }^{6} \mathrm{~S}$ in Re I.

The second step in the analysis of Tc I was taken by Bozman [3, 4], making use of the new and more extensive description of technetium spectra prepared in 1952 and recently published by Bozman, Meggers and Corliss [5]. In that step of the analysis Bozman made one of the first applications of electronic digital computers to the hitherto tedious work of searching out new energy levels. He found 37 new even and 42 odd energy levels, and classified about 900 lines.

In 1955 and 1956 Bozman observed the Zeeman Effect in Tc I by use of electrodeless lamps [6] containing technetium iodide. Hundreds of patterns were measured by him in the region between 2400 and $7000 \AA$ and a number of them were reduced to determine the magnetic splitting factors ( $g$-values). Bozman used some of these $g$-values to interpret

\footnotetext{
${ }^{1}$ Figures in brackets indicate the literature references at the end of this paper.
}

most of the Tc I levels published in Vol. III of "Atomic Energy Levels," compiled by C. E. Moore [7].

In the dozen years that have elapsed since the initial applications of digital computers to energy level searches, significant advances in programming this problem have been made, notably by G. Racah while visiting NBS and recently by one of the present authors [8]. In the currently undertaken third phase of the analysis of Tc I, Corliss and Tech have applied this latest program to a search for new energy levels.

\section{Search for New Energy Levels}

As the first step in discovering new energy levels in Tc I a special search was made with 61 even and 92 odd levels already known. These levels provided classifications for 914 lines which were then removed from the complete list of 3336 Tc I lines. A search with the remaining unclassified lines yielded 21 new odd levels. With these added to the odd level list, 29 new even levels were found. At this point, 623 more classified lines were removed from the line list. From a second pair of searches 35 odd and 20 even levels were found, classifying 569 lines. A third pair of searches revealed only 2 odd and 7 even levels, whereupon the systematic numerical searches were discontinued.

\section{Results}

The present lists of even and odd levels are given in tables 1 and 2 . The term designations assigned by Meggers or Bozman are given in the first column, the $J$-values in the second, and the level values in $\mathrm{cm}^{-1}$ in the third. The number of combinations with each level and the discoverer are given in the last column. Here "M" refers to Meggers (1951), "B" to Bozman 
and " $\mathrm{C}$ " to the levels recently found with the computer. "A" indicates that the level appears in AEL [7].

The list of classified lines with the wavelengths, wavenumbers, intensities, and energy levels is given in table 3. A doubly classified line is indicated by duplicate entries of the line in table 3 .

The intensity numbers are in some cases accompanied by literal symbols indicating characteristic features of the lines as follows:

$$
\begin{aligned}
& c \text {-complex } \\
& d \text {-double } \\
& h \text {-hazy. }
\end{aligned}
$$

l-shaded to longer waves

$s$-shaded to shorter waves

$w$-wide

tr-intensity $<1$.

About 2200 or two-thirds of the known Tc I lines are now classified.

The assignment of the Tc I energy levels to configurations and terms is proceeding with the reduction of the Zeeman patterns. This analysis, together with the results of theoretical calculations in progress, will appear in a later paper.

TABLE 1. Even levels of Tc I

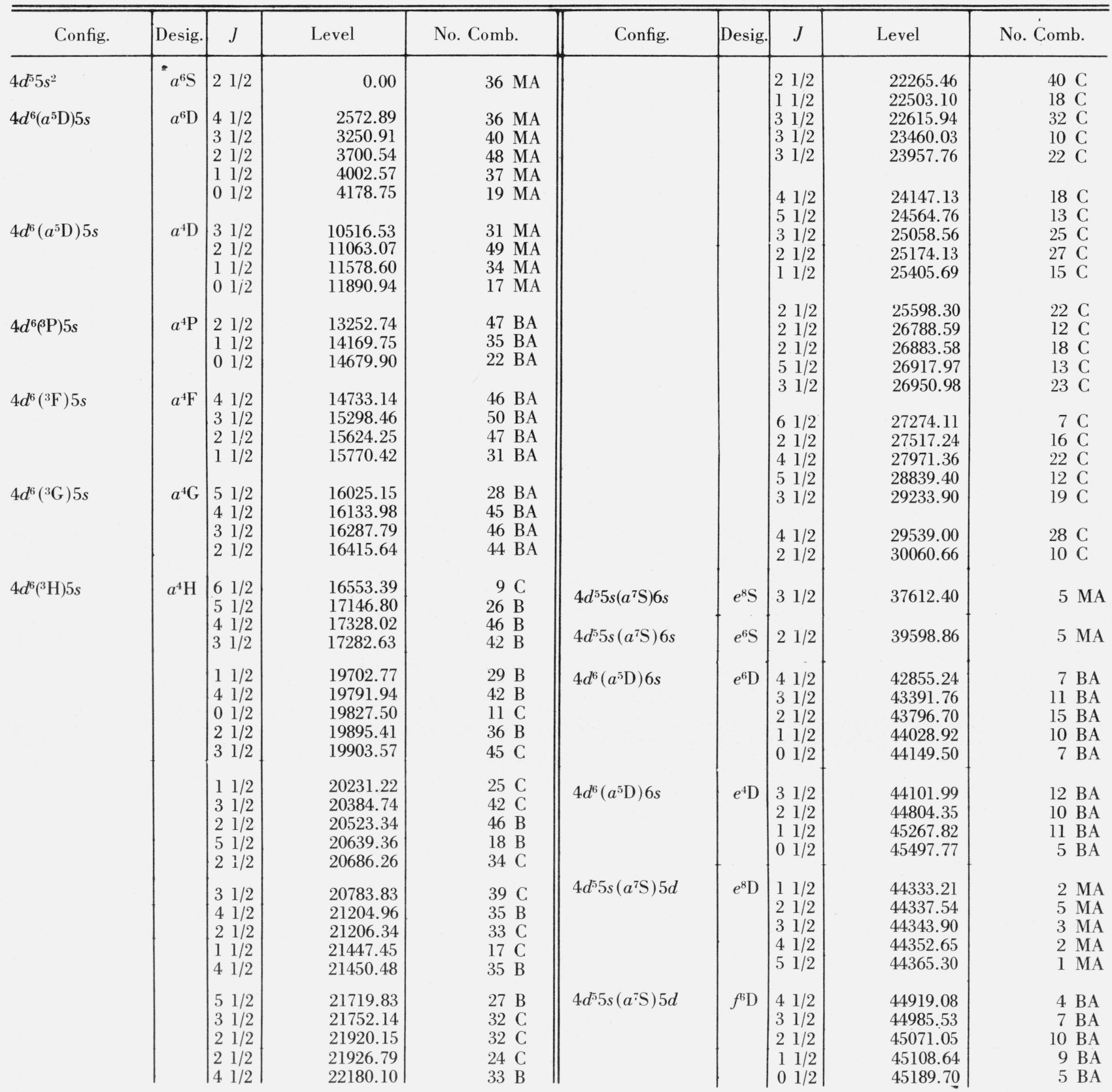


TABLE 1. Even levels of Te I-Continued

\begin{tabular}{|c|c|c|c|c|c|c|c|c|c|}
\hline Config. & Desig. & $J$ & Level & No. Comb. & Config. & Desig. & $J$ & Level & No. Comb. \\
\hline & & $\begin{array}{ll}4 & 1 / 2 \\
3 & 1 / 2 \\
3 & 1 / 2 \\
2 & 1 / 2 \\
4 & 1 / 2 \\
& \\
3 & 1 / 2 \\
2 & 1 / 2 \\
2 & 1 / 2 \\
3 & 1 / 2 \\
1 & 1 / 2\end{array}$ & $\begin{array}{l}48418.21 \\
48462.94 \\
48784.86 \\
48944.13 \\
48977.03 \\
\\
49245.93 \\
49337.51 \\
49565.96 \\
49573.35 \\
49735.08\end{array}$ & $\begin{array}{rl}7 & \mathrm{C} \\
5 & \mathrm{C} \\
10 & \mathrm{C} \\
11 & \mathrm{C} \\
9 & \mathrm{C} \\
& \\
9 & \mathrm{C} \\
7 & \mathrm{C} \\
8 & \mathrm{C} \\
5 & \mathrm{M} \\
11 & \mathrm{C}\end{array}$ & & & $\begin{array}{ll}2 & 1 / 2 \\
2 & 1 / 2 \\
2 & 1 / 2 \\
5 & 1 / 2 \\
5 & 1 / 2 \\
& \\
3 & 1 / 2 \\
3 & 1 / 2 \\
4 & 1 / 2 \\
2 & 1 / 2 \\
3 & 1 / 2\end{array}$ & $\begin{array}{l}49813.62 \\
49938.69 \\
53945.19 \\
56118.36 \\
59315.30 \\
\\
62038.26 \\
62501.78 \\
62599.48 \\
63926.66 \\
64785.90\end{array}$ & $\begin{array}{rl}10 & \mathrm{C} \\
10 & \mathrm{C} \\
6 & \mathrm{C} \\
5 & \mathrm{C} \\
7 & \mathrm{C} \\
7 & \mathrm{C} \\
8 & \mathrm{C} \\
7 & \mathrm{C} \\
7 & \mathrm{C} \\
7 & \mathrm{C}\end{array}$ \\
\hline
\end{tabular}

TABLE 2. Odd levels of Te I

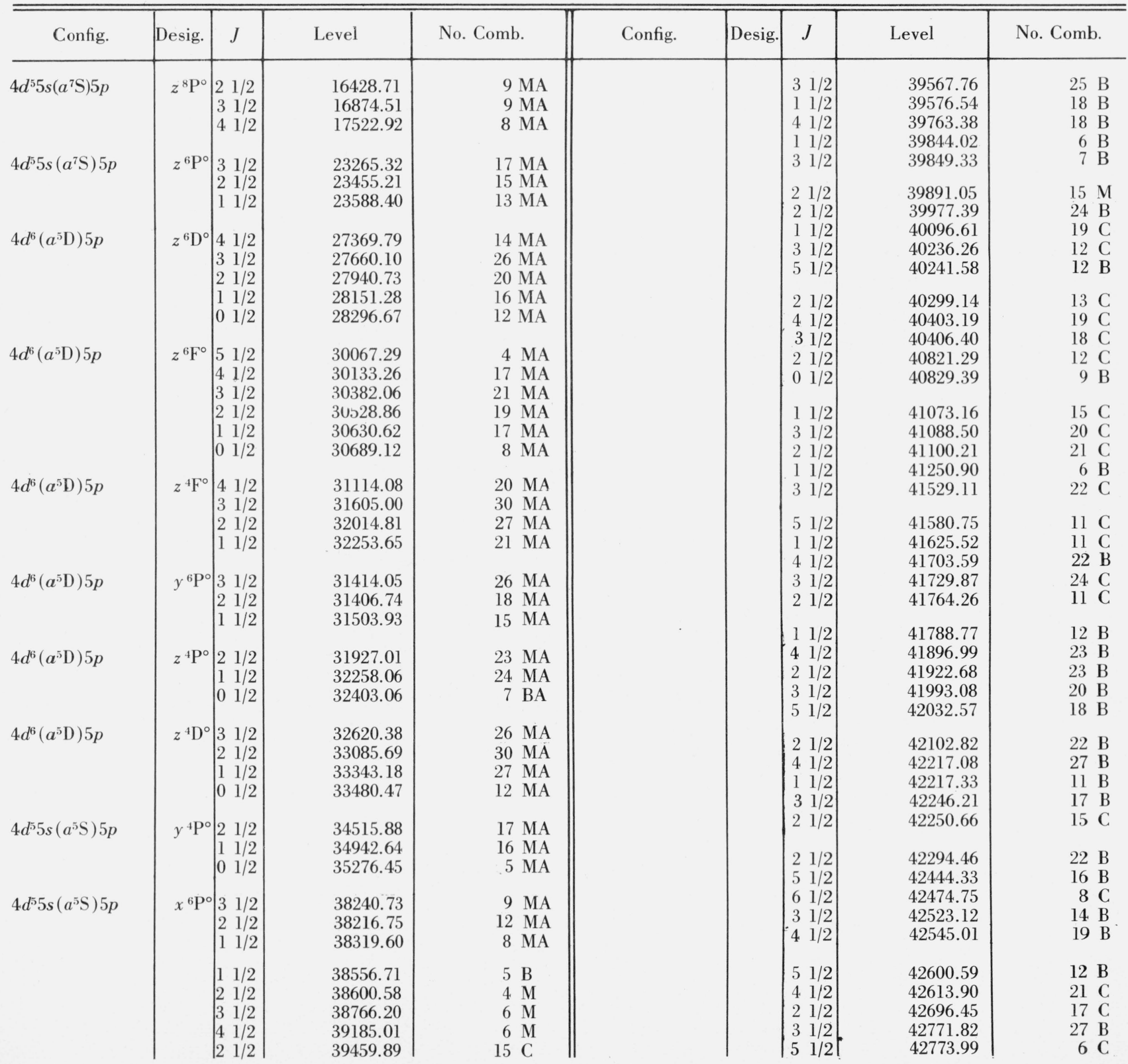


TABLE 2. Odd levels of Tc I-Continued

\begin{tabular}{|c|c|c|c|c|c|c|c|c|c|}
\hline Config. & Desig. & $J$ & Level & No. Comb. & Config. & Desig. & $J$ & Level & No. Comb. \\
\hline & & $\begin{array}{ll}4 & 1 / 2 \\
1 & 1 / 2 \\
2 & 1 / 2 \\
2 & 1 / 2 \\
3 & 1 / 2 \\
4 & 1 / 2 \\
5 & 1 / 2 \\
6 & 1 / 2 \\
4 & 1 / 2 \\
3 & 1 / 2 \\
& \\
5 & 1 / 2 \\
2 & 1 / 2 \\
4 & 1 / 2 \\
3 & 1 / 2 \\
6 & 1 / 2 \\
& \\
3 & 1 / 2 \\
4 & 1 / 2 \\
2 & 1 / 2 \\
3 & 1 / 2 \\
2 & 1 / 2 \\
& \\
1 & 1 / 2 \\
5 & 1 / 2 \\
2 & 1 / 2 \\
3 & 1 / 2 \\
1 & 1 / 2 \\
2 & 1 / 2 \\
4 & 1 / 2 \\
3 & 1 / 2 \\
1 & 1 / 2 \\
3 & 1 / 2 \\
\end{array}$ & $\begin{array}{l}42817.00 \\
42901.59 \\
42905.60 \\
43011.14 \\
43077.33 \\
\\
43097.77 \\
43172.19 \\
43197.60 \\
43265.51 \\
43267.16 \\
\\
43512.49 \\
43546.50 \\
43649.73 \\
43805.25 \\
43857.24 \\
\\
43905.73 \\
43971.81 \\
44007.00 \\
44090.55 \\
44107.18 \\
\\
44110.04 \\
44138.17 \\
44241.57 \\
44266.89 \\
44313.57 \\
44537.79 \\
44576.08 \\
44687.20 \\
44722.27 \\
44839.20\end{array}$ & $\begin{aligned} 18 \mathrm{~B} \\
14 \mathrm{C} \\
15 \mathrm{C} \\
21 \mathrm{C} \\
25 \mathrm{C} \\
15 \mathrm{C} \\
15 \mathrm{~B} \\
5 \mathrm{~B} \\
21 \mathrm{~B} \\
27 \mathrm{~B} \\
14 \mathrm{C} \\
15 \mathrm{C} \\
21 \mathrm{~B} \\
13 \mathrm{C} \\
8 \mathrm{C} \\
25 \mathrm{~B} \\
10 \mathrm{C} \\
18 \mathrm{C} \\
18 \mathrm{~B} \\
23 \mathrm{C} \\
15 \mathrm{C} \\
15 \mathrm{C} \\
18 \mathrm{C} \\
26 \mathrm{~B} \\
16 \mathrm{C} \\
17 \mathrm{~B} \\
20 \mathrm{C} \\
16 \mathrm{C} \\
14 \mathrm{~B} \\
18 \mathrm{C}\end{aligned}$ & & & $\begin{array}{ll}2 & 1 / 2 \\
1 & 1 / 2 \\
1 & 1 / 2 \\
4 & 1 / 2 \\
4 & 1 / 2 \\
& 1 / 2 \\
2 & 1 / 2 \\
3 & 1 / 2 \\
3 & 1 / 2 \\
3 & 1 / 2 \\
1 & 1 / 2 \\
2 & 1 / 2 \\
2 & 1 / 2 \\
5 & 1 / 2 \\
4 & 1 / 2 \\
3 & 1 / 2 \\
& \\
2 & 1 / 2 \\
0 & 1 / 2 \\
4 & 1 / 2 \\
3 & 1 / 2 \\
2 & 1 / 2 \\
4 & 1 / 2 \\
3 & 1 / 2 \\
5 & 1 / 2 \\
4 & 1 / 2 \\
4 & 1 / 2 \\
3 & 1 / 2 \\
4 & 1 / 2 \\
5 & 1 / 2 \\
3 & 1 / 2 \\
4 & 1 / 2\end{array}$ & $\begin{array}{l}44872.37 \\
44876.75 \\
44955.88 \\
45708.77 \\
46068.86 \\
46104.89 \\
46396.17 \\
46608.86 \\
46656.07 \\
47030.68 \\
47232.38 \\
47281.86 \\
47294.35 \\
47343.91 \\
47389.74 \\
47452.27 \\
47670.53 \\
47819.66 \\
47864.34 \\
48142.76 \\
48213.49 \\
48217.35 \\
48287.79 \\
48881.75 \\
49098.66 \\
49130.01 \\
49815.88 \\
51221.24 \\
51423.70 \\
52350.24\end{array}$ & $\begin{aligned} 16 \mathrm{C} \\
15 \mathrm{~B} \\
9 \mathrm{~B} \\
15 \mathrm{C} \\
13 \mathrm{C} \\
15 \mathrm{C} \\
14 \mathrm{C} \\
16 \mathrm{C} \\
17 \mathrm{C} \\
9 \mathrm{C} \\
14 \mathrm{C} \\
11 \mathrm{C} \\
8 \mathrm{C} \\
8 \mathrm{C} \\
11 \mathrm{C} \\
16 \mathrm{C} \\
5 \mathrm{~B} \\
16 \mathrm{C} \\
13 \mathrm{C} \\
11 \mathrm{C} \\
12 \mathrm{C} \\
11 \mathrm{~B} \\
11 \mathrm{C} \\
17 \mathrm{C} \\
10 \mathrm{C} \\
8 \mathrm{C} \\
12 \mathrm{C} \\
6 \mathrm{C} \\
10 \mathrm{C} \\
8 \mathrm{C}\end{aligned}$ \\
\hline
\end{tabular}


TABLE 3. Classified lines of TC I

\begin{tabular}{|c|c|c|c|c|}
\hline \multirow{2}{*}{$\begin{array}{c}\text { Wavelength } \\
\text { A }\end{array}$} & \multirow{2}{*}{$\underset{\mathrm{cm}^{-1}}{\text { Wavenumber }}$} & \multicolumn{2}{|c|}{ Intensity } & \multirow{2}{*}{$\begin{array}{c}\text { Energy levels } \\
\mathrm{cm}^{-1}\end{array}$} \\
\hline & & Arc & Spark & \\
\hline $\begin{array}{l}2154.668 \\
2271.662 \\
2272.976 \\
2282.121 \\
2405.128\end{array}$ & $\begin{array}{l}46396.27 \\
44007.03 \\
43981.59 \\
43805.36 \\
41565.18\end{array}$ & $\begin{array}{r}2 \\
3 \\
2 \\
10 \\
8\end{array}$ & & $\begin{array}{r}0_{2_{1 / 2}}-46396^{0} o_{3_{1 / 2}} \\
0_{2_{1 / 2}}-44007^{\circ} o_{2_{1 / 2}} \\
3250_{3_{1 / 2}}-47232^{\circ} o_{2_{1 / 2}} \\
0_{2_{1 / 2}}-43805^{\circ} o_{3_{1 / 2}} \\
2572_{4_{1 / 2}}-44138^{0} 5_{5_{1 / 2}}\end{array}$ \\
\hline $\begin{array}{l}2412.610 \\
2418.647 \\
2418.647 \\
2424.538 \\
2427.848\end{array}$ & $\begin{array}{l}41436.29 \\
41332.87 \\
41332.87 \\
41232.45 \\
41176.24\end{array}$ & $\begin{array}{r}6 \\
5 \\
5 \\
20 \\
3\end{array}$ & 1 & $\begin{array}{l}3250_{3_{1 / 2}}-44687^{0} o_{3_{1 / 2}} \\
25722_{4_{1 / 2}}-43905^{\circ} o_{3_{1 / 2}} \\
2572_{4_{1 / 2}}-43905^{\circ}{ }_{3_{1 / 2}} \\
2572_{4_{1 / 2}}-43805^{o}{ }_{3_{1 / 2}} \\
37000_{2_{1 / 2}}-44876^{o}{ }_{1_{1 / 2}}\end{array}$ \\
\hline $\begin{array}{l}2432.337 \\
2433.728 \\
2436.994 \\
2439.081 \\
2446.049\end{array}$ & $\begin{array}{l}41100.25 \\
41076.76 \\
41021.71 \\
40986.62 \\
40869.87\end{array}$ & $\begin{array}{r}2 \\
8 \\
10 \\
5 \\
3\end{array}$ & & $\begin{array}{r}0_{2_{1 / 2}}-41100^{o}{ }_{2_{1 / 2}} \\
2572_{4_{1 / 2}}-43649^{o}{ }_{{ }_{1 / 2}} \\
3700_{2_{1 / 2}}-44722^{o}{ }^{o}{ }_{1 / 2} \\
3700_{2_{1 / 2}}-44687^{\circ}{ }_{3_{1 / 2}} \\
4002_{1_{1 / 2}}-44872^{o}{ }_{{ }_{1 / 2}}\end{array}$ \\
\hline $\begin{array}{l}2446.864 \\
2447.860 \\
2456.386 \\
2458.994 \\
2461.523\end{array}$ & $\begin{array}{l}40856.26 \\
40839.63 \\
40697.89 \\
40654.73 \\
40612.96\end{array}$ & $\begin{array}{r}4 \\
3 \\
2 \\
10 \\
9\end{array}$ & & $\begin{array}{l}3250_{3_{1 / 2}}-44107^{0} o_{2_{1 / 2}} \\
3250_{3_{1 / 2}}-44090^{o} o_{3_{1 / 2}} \\
4178_{0_{1 / 2}}-44876^{o} o_{1_{1 / 2}} \\
3250_{3_{1 / 2}}-43905^{0}{ }_{3_{1 / 2}} \\
3700_{2_{1 / 2}}-44313^{0}{ }_{1_{1 / 2}}\end{array}$ \\
\hline $\begin{array}{l}2465.086 \\
2465.740 \\
2466.873 \\
2468.114 \\
2473.915\end{array}$ & $\begin{array}{l}40554.27 \\
40543.51 \\
40524.89 \\
40504.52 \\
40409.55\end{array}$ & $\begin{array}{r}20 \\
10 \\
30 \\
3 \\
8\end{array}$ & $\begin{array}{l}1 \\
1 \\
1\end{array}$ & $\begin{array}{l}3250_{3_{1 / 2}}-43805^{o}{ }_{3_{1 / 2}} \\
4178_{0_{1 / 2}}-44722^{o}{ }_{1_{1 / 2}} \\
2572_{4_{1 / 2}}-43097^{\circ}{ }_{4_{1 / 2}} \\
2572_{4_{1 / 2}}-43077^{o} 3_{3_{1 / 2}} \\
3700_{2_{1 / 2}}-44110^{o}{ }_{1_{1 / 2}}\end{array}$ \\
\hline $\begin{array}{l}2474.570 \\
2475.107 \\
2480.699 \\
2484.080 \\
2484.572\end{array}$ & $\begin{array}{l}40398.85 \\
40390.09 \\
40299.05 \\
40244.20 \\
40236.23\end{array}$ & $\begin{array}{r}2 \\
20 \\
50 \\
4 \\
8\end{array}$ & 8 & $\begin{array}{r}3250_{3_{1 / 2}}-43649^{o} a_{4_{1 / 2}} \\
3700_{2_{1 / 2}}-44090^{o} o_{3_{1 / 2}} \\
0_{2_{1 / 2}}-40299^{\circ} o_{2_{1 / 2}} \\
2572_{4_{1 / 2}}-42817^{\circ} o_{4_{1 / 2}} \\
0_{2_{1 / 2}}-40236^{o} o_{3_{1 / 2}}\end{array}$ \\
\hline $\begin{array}{l}2486.496 \\
2486.884 \\
2490.854 \\
2492.721 \\
2492.721\end{array}$ & $\begin{array}{l}40205.10 \\
40198.83 \\
40134.76 \\
40104.70 \\
40104.70\end{array}$ & $\begin{array}{r}20 \\
2 \\
10 \\
25 \\
25\end{array}$ & $\begin{array}{l}3 \\
3\end{array}$ & $\begin{array}{l}3700_{2_{1 / 2}}-43905^{0} o_{3_{1 / 2}} \\
2572_{4_{1 / 2}}-42771^{o} o_{3_{1 / 2}} \\
4178_{0_{1 / 2}}-44313^{o}{ }_{1_{1 / 2}} \\
3700_{2_{1 / 2}}-43805^{0}{ }_{3_{1 / 2}} \\
4002_{1_{1 / 2}}-44107^{0}{ }_{2_{1 / 2}}\end{array}$ \\
\hline $\begin{array}{l}2500.654 \\
2500.990 \\
2502.363 \\
2503.550 \\
2508.853\end{array}$ & $\begin{array}{l}39977.48 \\
39972.11 \\
39950.18 \\
39931.24 \\
39846.85\end{array}$ & $\begin{array}{r}2 \\
15 \\
7 \\
6 \\
10\end{array}$ & $\begin{array}{l}1 \\
1\end{array}$ & $\begin{array}{r}0_{2_{1 / 2}}-39977^{0} o_{2_{1 / 2}} \\
25722_{4_{1 / 2}}-42545^{\circ} o_{4_{1 / 2}} \\
25722_{4_{1 / 2}}-42523^{\circ}{ }_{3_{1 / 2}} \\
41788_{0_{1 / 2}}-44110^{o}{ }_{1_{1 / 2}} \\
3250_{3_{1 / 2}}-43097^{\circ}{ }_{1_{1 / 2}}\end{array}$ \\
\hline $\begin{array}{l}2521.679 \\
2526.548 \\
2529.550 \\
2533.467 \\
2538.804\end{array}$ & $\begin{array}{l}39644.19 \\
39567.79 \\
39520.84 \\
39459.74 \\
39376.79\end{array}$ & $\begin{array}{r}2 \\
3 \\
2 \\
10 \\
2\end{array}$ & 1 & $\begin{array}{r}2572_{4_{1 / 2}}-42217^{\circ} o_{4_{1 / 2}} \\
0_{2_{1 / 2}}-39567^{\circ} 3_{3_{1 / 2}} \\
3250_{3_{1 / 2}}-42771^{o} 3_{3_{1 / 2}} \\
2572_{4_{1 / 2}}-42032^{o} 5_{5_{1 / 2}} \\
3700_{2_{1 / 2}}-43077^{\circ} 3_{1_{1 / 2}}\end{array}$ \\
\hline $\begin{array}{l}2544.144 \\
2545.565 \\
2562.818 \\
2563.349 \\
2565.560\end{array}$ & $\begin{array}{l}39294.15 \\
39272.21 \\
39007.85 \\
38999.77 \\
38966.16\end{array}$ & $\begin{array}{r}2 \\
15 \\
10 \\
2 \\
4\end{array}$ & 1 & $\begin{array}{l}3250_{3_{1 / 2}}-42545^{0} a_{4_{1 / 2}} \\
3250_{3_{1 / 2}}-42523^{\circ} o_{3_{1 / 2}} \\
2572_{4_{1 / 2}}-41580^{\circ} o_{5_{1 / 2}} \\
3250_{3_{1 / 2}}-42250^{\circ} o_{2_{1 / 2}} \\
3250_{3_{1 / 2}}-42217^{\circ}{ }_{4_{1 / 2}}\end{array}$ \\
\hline
\end{tabular}


TABLE 3. Classified lines of TC I

\begin{tabular}{|c|c|c|c|c|}
\hline \multirow{2}{*}{$\begin{array}{c}\text { Wavelength } \\
\text { A }\end{array}$} & \multirow{2}{*}{$\begin{array}{l}\text { Wavenumber } \\
\mathrm{cm}^{-1}\end{array}$} & \multicolumn{2}{|c|}{ Intensity } & \multirow{2}{*}{$\begin{array}{l}\text { Energy levels } \\
\mathrm{cm}^{-1}\end{array}$} \\
\hline & & Arc & Spark & \\
\hline $\begin{array}{l}2578.791 \\
2589.861 \\
2592.816 \\
2593.247 \\
2605.753\end{array}$ & $\begin{array}{l}38766.25 \\
38600.56 \\
38556.57 \\
38550.16 \\
38365.16\end{array}$ & $\begin{array}{l}300 h l \\
200 \\
100 \\
10 \\
4\end{array}$ & $\begin{array}{c}100 h l \\
3 \\
5\end{array}$ & $\begin{array}{r}0_{2_{1 / 2}}-38766^{o}{ }_{3_{1 / 2}} \\
0_{2_{1 / 2}}-38600^{o}{ }_{2_{1 / 2}} \\
0_{2_{1 / 2}}-38556^{o}{ }_{1_{1 / 2}} \\
3700_{2_{1 / 2}}-42250^{0} o_{2_{1 / 2}} \\
10516_{3_{1 / 2}}-48881^{o}{ }_{4_{1 / 2}}\end{array}$ \\
\hline $\begin{array}{l}2608.855 \\
2614.233 \\
2615.873 \\
2642.372 \\
2654.306\end{array}$ & $\begin{array}{l}38319.54 \\
38240.72 \\
38216.74 \\
37833.51 \\
37663.42\end{array}$ & $\begin{array}{r}500 \\
1500 \\
1000 \\
100 \\
100\end{array}$ & $\begin{array}{r}15 \\
30 \\
20 \\
3\end{array}$ & $\begin{array}{r}0_{2_{1 / 2}}-38319^{o} o_{1 / 2} \\
0_{2_{1 / 2}}-38240^{o} o_{3_{1 / 2}} \\
0_{2_{1 / 2}}-38216^{o} o_{2_{1 / 2}} \\
2572_{4_{1 / 2}}-40406^{o} o_{3_{1 / 2}} \\
2572_{4_{1 / 2}}-40236^{o} o_{3_{1 / 2}}\end{array}$ \\
\hline $\begin{array}{l}2657.572 \\
2660.884 \\
2662.296 \\
2669.654 \\
2673.024\end{array}$ & $\begin{array}{l}37617.13 \\
37570.31 \\
37550.39 \\
37446.90 \\
37399.69\end{array}$ & $\begin{array}{r}1 \\
120 \\
100 \\
1 \\
6\end{array}$ & $\begin{array}{c}1 \\
30 l \\
2\end{array}$ & $\begin{array}{r}14733_{4_{1 / 2}}-52350^{o}{ }_{4_{1 / 2}} \\
3250_{3_{1 / 2}}-40821^{o}{ }_{{ }^{1 / 2}} \\
3700_{2_{1 / 2}}-41250^{0}{ }_{1_{1 / 2}} \\
4178_{0_{1 / 2}}-41625^{o} o_{1_{1 / 2}} \\
3700_{2_{1 / 2}}-41100^{0} o_{2_{1 / 2}}\end{array}$ \\
\hline $\begin{array}{l}2683.886 \\
2688.059 \\
2690.673 \\
2693.112 \\
2694.780\end{array}$ & $\begin{array}{l}37248.34 \\
37190.52 \\
37154.39 \\
37120.74 \\
37097.77\end{array}$ & $\begin{array}{l}80 \\
20 \\
12 \\
80 \\
10\end{array}$ & 1 & $\begin{array}{r}4002_{1_{1 / 2}}-41250^{o}{ }_{1_{1 / 2}} \\
2572_{4_{1 / 2}}-39763^{o}{ }_{4_{1 / 2}} \\
11063_{2_{1 / 2}}-48217^{\circ} o_{3_{1 / 2}} \\
3700_{2_{1 / 2}}-40821^{o} o_{2_{1 / 2}} \\
4002_{1_{1 / 2}}-41100^{\circ} o_{2_{1 / 2}}\end{array}$ \\
\hline $\begin{array}{l}2696.636 \\
2696.755 \\
2702.271 \\
2714.600 \\
2715.205\end{array}$ & $\begin{array}{l}37072.23 \\
37070.60 \\
36994.93 \\
36826.92 \\
36818.72\end{array}$ & $\begin{array}{r}50 \\
15 \\
70 \\
2 \\
30\end{array}$ & 1 & 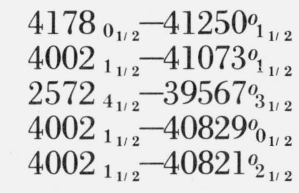 \\
\hline $\begin{array}{l}2723.550 \\
2730.529 \\
2736.231 \\
2737.973 \\
2739.819\end{array}$ & $\begin{array}{l}36705.91 \\
36612.10 \\
36535.81 \\
36512.56 \\
36487.96\end{array}$ & $\begin{array}{r}30 \\
500 \\
150 \\
100 \\
3\end{array}$ & $\begin{array}{c}10 \\
1\end{array}$ & $\begin{array}{r}3700_{2_{1 / 2}}-40406^{o} o_{3_{1 / 2}} \\
2572_{4_{1 / 2}}-39184^{o} 4_{1 / 2} \\
3700_{2_{1 / 2}}-40236^{o} o_{3_{1 / 2}} \\
3250_{3_{1 / 2}}-39763^{0} o_{4_{1 / 2}} \\
14733_{4_{1 / 2}}-51221^{0} \sigma_{5_{1 / 2}}\end{array}$ \\
\hline $\begin{array}{l}2755.761 \\
2760.380 \\
2762.134 \\
2762.337 \\
2763.957\end{array}$ & $\begin{array}{l}36276.89 \\
36216.19 \\
36193.19 \\
36190.53 \\
36169.32\end{array}$ & $\begin{array}{r}100 \\
7 \\
100 \\
200 \\
8\end{array}$ & $\begin{array}{l}8 \\
3 \\
8\end{array}$ & $\begin{array}{r}3700_{2_{1 / 2}}-39977^{0} o_{2_{1 / 2}} \\
16133_{4_{1 / 2}}-52350^{\circ}{ }^{\circ}{ }_{1 / 2} \\
2572_{4_{1 / 2}}-38766^{0} o_{3_{1 / 2}} \\
3700_{2_{1 / 2}}-39891^{\circ} o_{2_{1 / 2}} \\
11063_{2_{1 / 2}}-47232^{0}{ }_{2_{1 / 2}}\end{array}$ \\
\hline $\begin{array}{l}2769.714 \\
2778.908 \\
2782.052 \\
2785.586 \\
2787.234\end{array}$ & $\begin{array}{l}36094.15 \\
35974.74 \\
35934.08 \\
35888.50 \\
35867.28\end{array}$ & $\begin{array}{r}5 \\
150 \\
1000 \\
500 \\
15\end{array}$ & $\begin{array}{r}9 \\
15 \\
10 \\
2\end{array}$ & $\begin{array}{l}4002_{1_{1 / 2}}-40096^{0}{ }_{1_{1 / 2}} \\
4002_{1_{1 / 2}}-39977^{\circ}{ }_{2_{1 / 2}} \\
3250_{3_{1 / 2}}-39184^{\circ}{ }_{4_{1 / 2}} \\
4002_{1_{1 / 2}}-39891^{o}{ }_{2_{1 / 2}} \\
37000_{2_{1 / 2}}-39567^{\circ} 0_{3_{1 / 2}}\end{array}$ \\
\hline $\begin{array}{l}2789.246 \\
2795.650 \\
2802.810 \\
2803.018 \\
2803.921\end{array}$ & $\begin{array}{l}35841.41 \\
35759.31 \\
35667.96 \\
35665.32 \\
35653.83\end{array}$ & $\begin{array}{r}500 \\
80 \\
1000 \\
150 \\
8\end{array}$ & $\begin{array}{l}8 \\
3\end{array}$ & $\begin{array}{r}4002_{1_{1 / 2}}-39844^{o}{ }_{1_{1 / 2}} \\
37000_{2_{1 / 2}}-39459^{\circ} o_{2_{1 / 2}} \\
2572_{4_{1 / 2}}-38240^{0} o_{3_{1 / 2}} \\
4178_{0_{1 / 2}}-398444^{o} o_{1_{1 / 2}} \\
115788_{1_{1 / 2}}-47232_{2_{1 / 2}}^{o}\end{array}$ \\
\hline $\begin{array}{l}2810.225 \\
2812.447 \\
2814.860 \\
2819.460 \\
2828.042\end{array}$ & $\begin{array}{l}35573.86 \\
35545.75 \\
35515.28 \\
35457.34 \\
35349.75\end{array}$ & $\begin{array}{r}15 \\
3 \\
30 \\
40 \\
200\end{array}$ & $\begin{array}{r}1 \\
10\end{array}$ & $\begin{array}{r}4002_{1_{1 / 2}}-39576^{o}{ }_{1_{1 / 2}} \\
11063_{2_{1 / 2}}-46608^{o} o_{3_{1 / 2}} \\
3250_{3_{1 / 2}}-38766^{o} 0_{1 / 2} \\
4002_{1_{1 / 2}}-39459^{0} o_{2_{1 / 2}} \\
3250_{3_{1 / 2}}-38600^{0} o_{2_{1 / 2}}\end{array}$ \\
\hline
\end{tabular}


TABLE 3. Classified lines of T $\mathrm{T}$

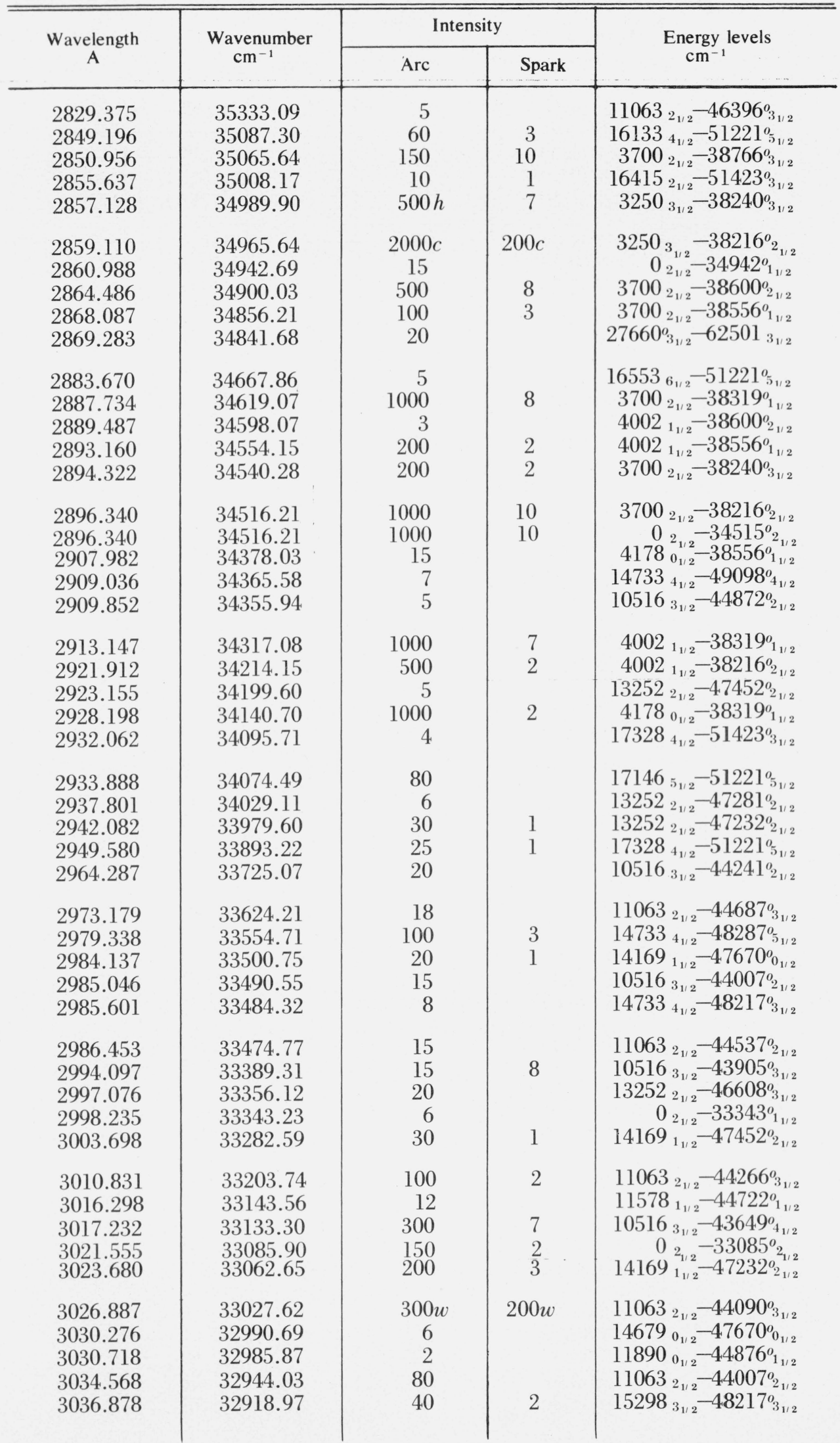


TABLE 3. Classified lines of TC I

\begin{tabular}{|c|c|c|c|c|}
\hline \multirow{2}{*}{$\begin{array}{c}\text { Wavelength } \\
\mathrm{A}\end{array}$} & \multirow{2}{*}{$\begin{array}{c}\text { Wavenumber } \\
\mathrm{cm}^{-1}\end{array}$} & \multicolumn{2}{|c|}{ Intensity } & \multirow{2}{*}{$\underset{\mathrm{cm}^{-1}}{\text { Energy levels }}$} \\
\hline & & Arc & Spark & \\
\hline $\begin{array}{l}3042.643 \\
3043.049 \\
3043.774 \\
3043.938 \\
3044.978\end{array}$ & $\begin{array}{l}32856.60 \\
32852.22 \\
32844.39 \\
32842.62 \\
32831.40\end{array}$ & $\begin{array}{r}40 \\
12 \\
8 \\
8 \\
10\end{array}$ & $\begin{array}{l}2 \\
1\end{array}$ & $\begin{array}{l}160255_{5_{1 / 2}}-48881_{4_{1 / 2}}^{o_{1 / 2}} \\
13252_{2_{1 / 2}}-461040_{2_{1 / 2}}^{o} \\
15298_{3_{1 / 2}}-48142^{o_{1 / 2}} \\
11063_{2_{1 / 2}}-43905^{o} o_{3_{1 / 2}} \\
11890_{0_{1 / 2}}-44722^{o} o_{1_{1 / 2}}\end{array}$ \\
\hline $\begin{array}{l}3057.327 \\
3060.103 \\
3060.672 \\
3061.283 \\
3064.667\end{array}$ & $\begin{array}{l}32698.80 \\
32669.14 \\
32663.06 \\
32656.54 \\
32620.49\end{array}$ & $\begin{array}{r}30 \\
4 \\
30 \\
3 \\
300\end{array}$ & $\begin{array}{l}2 \\
1\end{array}$ & $\begin{array}{r}168740_{3_{1 / 2}}-49573_{3_{1 / 2}} \\
17146_{5_{1 / 2}}-49815^{o} a_{1 / 2} \\
115788_{1_{1 / 2}}-44241_{2_{1 / 2}}^{o} \\
14733_{4_{1 / 2}}-47389^{o} o_{3_{1 / 2}} \\
0_{2_{1 / 2}}-32620^{o} o_{3_{1 / 2}}\end{array}$ \\
\hline $\begin{array}{l}3070.241 \\
3073.046 \\
3073.326 \\
3074.270 \\
3076.528\end{array}$ & $\begin{array}{l}32561.27 \\
32531.55 \\
32528.58 \\
32518.60 \\
32494.73\end{array}$ & $\begin{array}{l}30 \\
10 \\
12 \\
20 \\
10\end{array}$ & 1 & $\begin{array}{l}147333_{4_{1 / 2}}-47294^{o} o_{5_{1 / 2}} \\
115788_{1_{1 / 2}}-44110^{o}{ }_{1_{1 / 2}} \\
115788_{1_{1 / 2}}-44107^{\circ} o_{1 / 2} \\
15624{ }_{2_{1 / 2}}-48142^{o} o_{2_{1 / 2}} \\
10516_{3_{1 / 2}}-43011_{2_{1 / 2}}^{o}\end{array}$ \\
\hline $\begin{array}{l}3077.591 \\
3081.075 \\
3083.354 \\
3098.657 \\
3099.098\end{array}$ & $\begin{array}{l}32483.51 \\
32446.78 \\
32422.79 \\
32262.68 \\
32258.09\end{array}$ & $\begin{array}{r}3 \\
15 \\
20 \\
20 \\
1000\end{array}$ & 10 & $\begin{array}{r}110633_{2_{1 / 2}}-43546^{o} o_{2_{1 / 2}} \\
199033_{3_{1 / 2}}-52350^{\circ} o_{4_{1 / 2}} \\
31503^{\circ}{ }_{1_{1 / 2}}-63926^{a_{1 / 2}} \\
160255_{5_{1 / 2}}-48287^{\circ} 5_{5_{1 / 2}} \\
0_{2_{1 / 2}}-32258^{o} o_{1_{1 / 2}}\end{array}$ \\
\hline $\begin{array}{l}3099.517 \\
3104.293 \\
3109.151 \\
3115.977 \\
3119.171\end{array}$ & $\begin{array}{l}32253.73 \\
32204.11 \\
32153.79 \\
32083.35 \\
32050.50\end{array}$ & $\begin{array}{r}200 \\
30 \\
40 \\
60 \\
80\end{array}$ & $\begin{array}{l}2 \\
1\end{array}$ & $\begin{array}{r}0_{2_{1 / 2}}-32253^{o} o_{1_{1 / 2}} \\
110633_{2_{1 / 2}}-43267^{\circ} 0_{3_{1 / 2}} \\
161333_{4_{1 / 2}}-48287^{\circ} 5^{5_{1 / 2}} \\
161333_{4_{1 / 2}}-48217^{\circ} 3_{3_{1 / 2}} \\
17522^{o} 4_{1 / 2}-495733_{3_{1 / 2}}\end{array}$ \\
\hline $\begin{array}{l}3119.662 \\
3121.306 \\
3122.642 \\
3127.464 \\
3131.233\end{array}$ & $\begin{array}{l}32045.46 \\
32028.58 \\
32014.88 \\
31965.52 \\
31927.04\end{array}$ & $\begin{array}{c}40 \\
10 h \\
700 \\
2 \\
1500\end{array}$ & $\begin{array}{r}1 \\
3 \\
20\end{array}$ & $\begin{array}{r}15298_{3_{1 / 2}}-47343^{\circ}{ }_{4_{1 / 2}} \\
10516_{3_{1 / 2}}-42545^{\circ}{ }_{4_{1 / 2}} \\
0_{2_{1 / 2}}-32014^{\circ}{ }_{2_{1 / 2}} \\
20384_{3_{1 / 2}}-52350^{\circ}{ }_{4_{1 / 2}} \\
0_{2_{1 / 2}}-31927^{\circ}{{ }_{2}}_{2_{1 / 2}}\end{array}$ \\
\hline $\begin{array}{l}3138.322 \\
3139.936 \\
3143.561 \\
3144.291 \\
3145.937\end{array}$ & $\begin{array}{l}31854.93 \\
31838.55 \\
31801.84 \\
31794.46 \\
31777.82\end{array}$ & $\begin{array}{r}20 \\
25 \\
4 \\
20 \\
6\end{array}$ & & $\begin{array}{l}16287_{3_{1 / 2}}-48142^{o} o_{2_{1 / 2}} \\
110633_{2_{1 / 2}}-42901^{o} o_{1 / 2} \\
164155_{2_{1 / 2}}-48217^{\circ} 0_{3_{1 / 2}} \\
160255_{5_{1 / 2}}-47819^{\circ}{ }_{a_{1 / 2}} \\
10516_{3_{1 / 2}}-42294^{o} o_{2_{1 / 2}}\end{array}$ \\
\hline $\begin{array}{l}3147.160 \\
3150.265 \\
3150.650 \\
3152.779 \\
3153.353\end{array}$ & $\begin{array}{l}31765.48 \\
31734.17 \\
31730.29 \\
31708.86 \\
31703.09\end{array}$ & $\begin{array}{l}15 \\
40 c \\
8 \\
3 \\
20\end{array}$ & $\begin{array}{l}1 \\
5 c\end{array}$ & $\begin{array}{l}156244_{2_{1 / 2}}-47389^{o}{ }_{3_{1 / 2}} \\
10516_{3_{1 / 2}}-42250^{\circ} o_{2_{1 / 2}} \\
161333_{4_{1 / 2}}-47864^{o} 3_{1 / 2} \\
110632_{2_{1 / 2}}-42771^{o} o_{3_{1 / 2}} \\
13252_{2_{1 / 2}}-44955^{\circ} o_{1_{1 / 2}}\end{array}$ \\
\hline $\begin{array}{l}3155.079 \\
3155.476 \\
3157.339 \\
3160.463 \\
3161.674\end{array}$ & $\begin{array}{l}31685.75 \\
31681.76 \\
31663.07 \\
31631.77 \\
31619.66\end{array}$ & $\begin{array}{r}2 \\
5 \\
5 \\
2 \\
300\end{array}$ & 8 & $\begin{array}{l}161333_{4_{1 / 2}}-47819^{o}{ }_{4_{1 / 2}} \\
15770{ }_{1_{1 / 2}}-47452^{\circ}{ }_{2_{1 / 2}} \\
14733_{4_{1 / 2}}-46396^{o}{ }_{3_{1 / 2}} \\
19791_{4_{1 / 2}}-51423^{o} o_{3_{1 / 2}} \\
13252_{2_{1 / 2}}-44872^{o} o_{2_{1 / 2}}\end{array}$ \\
\hline $\begin{array}{l}3163.140 \\
3165.003 \\
3166.993 \\
3168.271 \\
3170.484\end{array}$ & $\begin{array}{l}31605.00 \\
31586.40 \\
31566.55 \\
31553.82 \\
31531.80\end{array}$ & $\begin{array}{r}12 \\
6 \\
3 \\
1 \\
1\end{array}$ & & $\begin{array}{r}0_{2_{1 / 2}}-31605^{o} o_{3_{1 / 2}} \\
10516_{3_{1 / 2}}-42102^{o_{2_{1 / 2}}} \\
20783_{3_{1 / 2}}-52350^{\circ}{ }_{4_{1 / 2}} \\
17328_{4_{1 / 2}}-48881^{\circ}{ }_{4_{1 / 2}} \\
16287_{3_{1 / 2}}-47819^{\circ}{ }_{4_{1 / 2}}\end{array}$ \\
\hline
\end{tabular}


TABLE 3. Classified lines of Tc I

\begin{tabular}{|c|c|c|c|c|}
\hline \multirow{2}{*}{$\begin{array}{c}\text { Wavelength } \\
\text { A }\end{array}$} & \multirow{2}{*}{$\begin{array}{l}\text { Wavenumber } \\
\mathrm{cm}^{-1}\end{array}$} & \multicolumn{2}{|c|}{ Intensity } & \multirow{2}{*}{$\begin{array}{l}\text { Energy levels } \\
\mathrm{cm}^{-1}\end{array}$} \\
\hline & & Arc & Spark & \\
\hline $\begin{array}{l}3170.833 \\
3173.295 \\
3177.714 \\
3180.305 \\
3182.367\end{array}$ & $\begin{array}{l}31528.33 \\
31503.87 \\
31460.06 \\
31434.43 \\
31414.06\end{array}$ & $\begin{array}{r}3 \\
3000 \\
6 \\
200 \\
2000\end{array}$ & $\begin{array}{r}100 \\
3 \\
60\end{array}$ & $\begin{array}{r}19895_{2_{1 / 2}}-51423^{o_{3_{1 / 2}}} \\
02_{2_{1 / 2}}-31503^{o_{1_{1 / 2}}} \\
11063_{2_{1 / 2}}-42523^{o_{3_{1 / 2}}} \\
13252_{2_{1 / 2}}-44687^{o_{3_{1 / 2}}} \\
0_{2_{1 / 2}}-314144^{o_{3_{1 / 2}}}\end{array}$ \\
\hline $\begin{array}{l}3183.108 \\
3191.620 \\
3192.051 \\
3192.905 \\
3195.485\end{array}$ & $\begin{array}{l}31406.75 \\
31322.99 \\
31318.76 \\
31310.38 \\
31285.11\end{array}$ & $\begin{array}{r}2000 \\
10 \\
20 \\
2 \\
20\end{array}$ & 60 & $\begin{array}{r}0_{2_{1 / 2}}-31406^{0} o_{2_{1 / 2}} \\
115788_{1_{1 / 2}}-42901^{0}{ }_{1_{1 / 2}} \\
16025_{5_{1 / 2}}-47343^{0}{ }_{4_{1 / 2}} \\
15298_{3_{1 / 2}}-46608^{0}{ }_{3_{1 / 2}} \\
13252_{2_{1 / 2}}-44537^{0} 0_{2_{1 / 2}}\end{array}$ \\
\hline $\begin{array}{l}3197.530 \\
3198.497 \\
3200.980 \\
3202.831 \\
3205.474\end{array}$ & $\begin{array}{l}31265.10 \\
31255.65 \\
31231.40 \\
31213.35 \\
31187.62\end{array}$ & $\begin{array}{c}40 w \\
15 \\
8 \\
300 c \\
4\end{array}$ & $\begin{array}{l}3 \\
6 c\end{array}$ & $\begin{array}{r}3250_{3_{1 / 2}}-34515^{o} o_{2_{1 / 2}} \\
161333_{4_{1 / 2}}-47389^{\circ}{ }_{3_{1 / 2}} \\
110633_{2_{1 / 2}}-422944^{o} o_{1 / 2} \\
10516_{3_{1 / 2}}-41729^{\circ}{ }_{3_{1 / 2}} \\
110633_{2_{1 / 2}}-42250^{\circ} o_{2_{1 / 2}}\end{array}$ \\
\hline $\begin{array}{l}3207.866 \\
3208.270 \\
3208.904 \\
3209.838 \\
3214.738\end{array}$ & $\begin{array}{l}31164.36 \\
31160.44 \\
31154.28 \\
31145.22 \\
31097.75\end{array}$ & $\begin{array}{l}4 \\
6 \\
5 \\
1 \\
3\end{array}$ & & $\begin{array}{r}16287_{3_{1 / 2}}-47452^{o} o_{2_{1 / 2}} \\
161333_{4_{1 / 2}}-47294^{o} o_{5_{1 / 2}} \\
110632_{2_{1 / 2}}-42217^{0}{ }_{1 / 2} \\
212044_{4_{1 / 2}}-52350^{o}{ }_{4_{1 / 2}} \\
41788_{0_{1 / 2}}-35276_{0_{1 / 2}}^{o}\end{array}$ \\
\hline $\begin{array}{l}3215.764 \\
3217.956 \\
3218.559 \\
3219.052 \\
3220.737\end{array}$ & $\begin{array}{l}31087.83 \\
31066.65 \\
31060.83 \\
31056.07 \\
31039.83\end{array}$ & $\begin{array}{r}3 \\
2 \\
10 \\
3 \\
40\end{array}$ & 2 & $\begin{array}{l}314144_{3_{1 / 2}}^{o}-62501_{3_{1 / 2}} \\
171466_{5_{1 / 2}}-48213^{o}{ }_{4_{1 / 2}} \\
13252_{2_{1 / 2}}-44313^{o}{ }_{1 / 2} \\
16287_{3_{1 / 2}}-47343^{o}{ }_{4_{1 / 2}} \\
11063_{2_{1 / 2}}-42102^{o} o_{1 / 2}\end{array}$ \\
\hline $\begin{array}{l}3221.573 \\
3223.762 \\
3225.500 \\
3226.044 \\
3227.412\end{array}$ & $\begin{array}{l}31031.77 \\
31010.70 \\
30993.99 \\
30988.77 \\
30975.63\end{array}$ & $\begin{array}{r}1 \\
3 \\
2 \\
15 \\
4\end{array}$ & & $\begin{array}{l}156244_{2_{1 / 2}}-46656^{o}{ }_{3_{1 / 2}} \\
11890_{0_{1 / 2}}-42901^{o}{ }_{1_{1 / 2}} \\
16287_{3_{1 / 2}}-47281^{o_{1 / 2}} \\
13252_{2_{1 / 2}}-44241^{o_{1 / 2}} \\
14733_{4_{1 / 2}}-45708^{o} o_{4_{1 / 2}}\end{array}$ \\
\hline $\begin{array}{l}3230.640 \\
3231.114 \\
3236.439 \\
3239.789 \\
3241.837\end{array}$ & $\begin{array}{l}30944.68 \\
30940.14 \\
30889.24 \\
30857.30 \\
30837.81\end{array}$ & $\begin{array}{r}1 \\
15 \\
5 \\
5 \\
100\end{array}$ & 5 & $\begin{array}{r}16287_{3_{1 / 2}}-47232^{o} o_{2_{1 / 2}} \\
40022_{1_{1 / 2}}-34942^{o}{ }_{1_{1 / 2}} \\
17328{ }_{4_{1 / 2}}-48217^{0}{ }^{0} 3_{1 / 2} \\
13252_{2_{1 / 2}}-44110^{o}{ }_{1_{1 / 2}} \\
13252_{2_{1 / 2}}-44090^{o} o_{3_{1 / 2}}\end{array}$ \\
\hline $\begin{array}{l}3244.193 \\
3249.600 \\
3250.638 \\
3252.048 \\
3254.700\end{array}$ & $\begin{array}{l}30815.41 \\
30764.14 \\
30754.32 \\
30740.98 \\
30715.93\end{array}$ & $\begin{array}{r}500 \\
10 \\
10 \\
300 \\
10\end{array}$ & 15 & 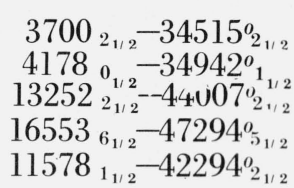 \\
\hline $\begin{array}{l}3256.102 \\
3259.261 \\
3259.910 \\
3261.370 \\
3262.896\end{array}$ & $\begin{array}{l}30702.71 \\
30672.95 \\
30666.85 \\
30653.12 \\
30638.78\end{array}$ & $\begin{array}{r}40 \\
3 \\
10 \\
20 \\
6\end{array}$ & $\begin{array}{l}2 \\
1 \\
5 \\
3 \\
2 h\end{array}$ & $\begin{array}{l}14169_{1_{1 / 2}}-44872^{o} o_{2_{1 / 2}} \\
171466_{5_{1 / 2}}-47819^{o}{ }_{4_{1 / 2}} \\
11063_{2_{1 / 2}}-41729^{o}{ }^{o}{ }^{3_{1 / 2}} \\
13252_{2_{1 / 2}}-43905^{o} o_{3_{1 / 2}} \\
11578_{1_{1 / 2}}-4217^{0} o_{1_{1 / 2}}\end{array}$ \\
\hline $\begin{array}{l}3272.111 \\
3276.29 \\
3281.392 \\
3291.960 \\
3296.504\end{array}$ & $\begin{array}{l}30552.50 \\
30513.54 \\
30466.09 \\
30368.29 \\
30326.43\end{array}$ & $\begin{array}{r}10 \\
5 \\
6 \\
20 \\
10\end{array}$ & 2 & $\begin{array}{r}14169_{1_{1 / 2}}-44722^{o}{ }_{1_{1 / 2}} \\
40022_{1_{1 / 2}}-34515^{o}{ }_{{ }_{1 / 2}} \\
110633_{2_{1 / 2}}-41529^{o}{ }_{3_{1 / 2}} \\
16287_{3_{1 / 2}}-46656^{o}{ }_{3_{1 / 2}} \\
11890_{0_{1 / 2}}-4217^{0} o_{1_{1 / 2}}\end{array}$ \\
\hline
\end{tabular}


TABLE 3. Classified lines of TC I

\begin{tabular}{|c|c|c|c|c|}
\hline \multirow{2}{*}{$\begin{array}{c}\text { Wavelength } \\
\text { A }\end{array}$} & \multirow{2}{*}{$\begin{array}{l}\text { Wavenumber } \\
\mathrm{cm}^{-1}\end{array}$} & \multicolumn{2}{|c|}{ Intensity } & \multirow{2}{*}{$\begin{array}{l}\text { Energy levels } \\
\mathrm{cm}^{-1}\end{array}$} \\
\hline & & Arc & Spark & \\
\hline $\begin{array}{l}3297.074 \\
3301.990 \\
3303.510 \\
3305.891 \\
3310.646\end{array}$ & $\begin{array}{l}30321.19 \\
30276.05 \\
30262.12 \\
30240.32 \\
30196.89\end{array}$ & $\begin{array}{r}3 \\
20 \\
6 \\
80 \\
200\end{array}$ & $\begin{array}{l}10 \\
15\end{array}$ & $\begin{array}{l}162877_{3_{1 / 2}}-46608^{o}{ }_{3_{1 / 2}} \\
14679_{0_{1 / 2}}-44955^{o} 0_{1_{1 / 2}} \\
16133_{4_{1 / 2}}-46396^{o} 3_{3_{1 / 2}} \\
164155_{2_{1 / 2}}-46656^{o} 3_{3_{1 / 2}} \\
14679_{0_{1 / 2}}-44876^{o}{ }_{1_{1 / 2}}\end{array}$ \\
\hline $\begin{array}{l}3311.051 \\
3313.652 \\
3327.102 \\
3327.665 \\
3328.252\end{array}$ & $\begin{array}{l}30193.20 \\
30169.50 \\
30047.54 \\
30042.46 \\
30037.16\end{array}$ & $\begin{array}{l}20 \\
150 \\
150 \mathrm{cw} \\
1 \\
5\end{array}$ & $\begin{array}{l}2 \\
5 \\
1 c\end{array}$ & $\begin{array}{r}16415_{2_{1 / 2}}-46608^{o}{ }_{3_{1 / 2}} \\
17282_{3_{1 / 2}}-47452^{\circ}{ }_{2_{1 / 2}} \\
2572_{4_{1 / 2}}-32620^{\circ}{ }^{o}{ }_{1 / 2} \\
14679_{0_{1 / 2}}-44722^{\circ}{ }_{1 / 2} \\
11063_{2_{1 / 2}}-41100^{\circ}{ }_{2_{1 / 2}}\end{array}$ \\
\hline $\begin{array}{l}3330.766 \\
3332.468 \\
3337.486 \\
3337.977 \\
3339.332\end{array}$ & $\begin{array}{l}30014.49 \\
29999.16 \\
29954.05 \\
29949.65 \\
29937.50\end{array}$ & $\begin{array}{r}100 \\
50 \\
10 \\
5 \\
6\end{array}$ & $\begin{array}{l}2 \\
2 \\
2 h l\end{array}$ & 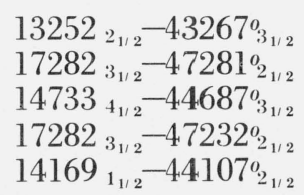 \\
\hline $\begin{array}{l}3342.141 \\
3343.770 \\
3345.016 \\
3350.563 \\
3350.830\end{array}$ & $\begin{array}{l}29912.34 \\
29897.76 \\
29886.63 \\
29837.15 \\
29834.77\end{array}$ & $\begin{array}{l}3 \\
10 \\
15 w \\
60 \\
50 c\end{array}$ & $\begin{array}{l}3 \\
1 h\end{array}$ & $\begin{array}{r}19903_{3_{1 / 2}}-4.9815^{o}{ }_{4_{1 / 2}} \\
11890_{0_{1 / 2}}-41788^{o}{ }_{1_{1 / 2}} \\
10516_{3_{1 / 2}}-40403^{o}{ }_{4_{1 / 2}} \\
14169_{1_{1 / 2}}-44007^{\circ} o_{2_{1 / 2}} \\
3250_{3_{1 / 2}}-33085^{\circ} o_{2_{1 / 2}}\end{array}$ \\
\hline $\begin{array}{l}3351.985 \\
3356.884 \\
3358.038 \\
3362.156 \\
3371.402\end{array}$ & $\begin{array}{l}29824.49 \\
29780.97 \\
29770.73 \\
29734.27 \\
29652.73\end{array}$ & $\begin{array}{r}1 \\
8 \\
12 \\
1 \\
5\end{array}$ & & $\begin{array}{l}13252_{2_{1 / 2}}-43077^{o} o_{3_{1 / 2}} \\
16287_{3_{1 / 2}}-46068^{\circ}{ }_{4_{1 / 2}} \\
21450_{4_{1 / 2}}-51221^{o}{ }_{5_{1 / 2}} \\
22615_{3_{1 / 2}}-52350^{\circ}{ }_{{ }_{1 / 2}} \\
13252_{2_{1 / 2}}-42905^{o} o_{2_{1 / 2}}\end{array}$ \\
\hline $\begin{array}{l}3372.543 \\
3373.572 \\
3386.387 \\
3386.674 \\
3388.459\end{array}$ & $\begin{array}{l}29642.70 \\
29633.66 \\
29521.52 \\
29519.02 \\
29503.47\end{array}$ & $\begin{array}{r}8 \\
25 \\
20 \\
40 \\
8\end{array}$ & 1 & $\begin{array}{r}3700_{2_{1 / 2}}-33343^{o}{ }_{1_{1 / 2}} \\
14679_{0_{1 / 2}}-44313^{o}{ }_{1_{1 / 2}} \\
11578_{1_{1 / 2}}-41100^{o}{ }_{{ }_{1 / 2}} \\
13252_{2_{1 / 2}}-42771^{o} o_{3_{1 / 2}} \\
21920_{2_{1 / 2}}-51423^{o} o_{3_{1 / 2}}\end{array}$ \\
\hline $\begin{array}{l}3389.492 \\
3396.903 \\
3398.327 \\
3401.681 \\
3402.105\end{array}$ & $\begin{array}{l}29494.47 \\
29430.13 \\
29417.80 \\
29388.79 \\
29385.13\end{array}$ & $\begin{array}{r}12 \\
60 \\
300 \\
25 \\
200\end{array}$ & $\begin{array}{l}1 \\
6 \\
1 \\
4\end{array}$ & $\begin{array}{r}11578_{1_{1 / 2}}-41073^{o}{ }_{1_{1 / 2}} \\
14679_{0_{1 / 2}}-44110^{o}{ }_{1_{1 / 2}} \\
32620^{o} o_{1 / 2}-62038_{3_{1 / 2}} \\
15298_{3_{1 / 2}}-44687^{\circ} o_{3_{1 / 2}} \\
3700_{2_{1 / 2}}-33085^{o} o_{2_{1 / 2}}\end{array}$ \\
\hline $\begin{array}{l}3403.330 \\
3403.931 \\
3405.328 \\
3406.964 \\
3407.281\end{array}$ & $\begin{array}{l}29374.55 \\
29369.37 \\
29357.32 \\
29343.22 \\
29340.49\end{array}$ & $\begin{array}{c}3 \\
200 c \\
80 \\
10 \\
80\end{array}$ & $\begin{array}{l}2 \\
5\end{array}$ & 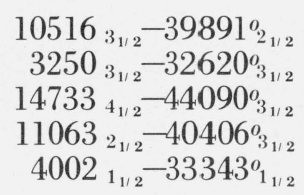 \\
\hline $\begin{array}{l}3411.804 \\
3414.600 \\
3415.448 \\
3417.738 \\
3418.053\end{array}$ & $\begin{array}{l}29301.60 \\
29277.60 \\
29270.34 \\
29250.72 \\
29248.03\end{array}$ & $\begin{array}{l}40 \\
10 \\
15 \\
10 \\
20\end{array}$ & 1 & $\begin{array}{r}4178_{0_{1 / 2}}-33480^{o} 0_{1 / 2} \\
15298_{3_{1 / 2}}-44576^{o}{ }_{{ }_{1 / 2}} \\
13252_{2_{1 / 2}}-42523^{o} o_{3_{1 / 2}} \\
115788_{1_{1 / 2}}-40829^{\circ} o_{0_{1 / 2}} \\
15624_{2_{1 / 2}}-44872^{o} o_{2_{1 / 2}}\end{array}$ \\
\hline $\begin{array}{l}3418.203 \\
3418.581 \\
3419.451 \\
3420.583 \\
3425.382\end{array}$ & $\begin{array}{l}29246.75 \\
29243.51 \\
29236.07 \\
29226.40 \\
29185.45\end{array}$ & $\begin{array}{l}40 c w \\
15 \\
15 \\
2 \\
20\end{array}$ & 1 & $\begin{array}{l}10516_{3_{1 / 2}}-39763^{o}{ }_{4_{1 / 2}} \\
22180_{4_{1 / 2}}-51423^{o} o_{3_{1 / 2}} \\
11063_{2_{1 / 2}}-40299^{\circ}{ }_{2_{1 / 2}} \\
19903_{3_{1 / 2}}-49130^{\circ}{ }_{3_{1 / 2}} \\
15770_{1_{1 / 2}}-44955^{\circ}{ }_{1_{1 / 2}}\end{array}$ \\
\hline
\end{tabular}


TABLE 3. Classified lines of Tc I

\begin{tabular}{|c|c|c|c|c|}
\hline \multirow{2}{*}{$\begin{array}{l}\text { Wavelength } \\
\text { A }\end{array}$} & \multirow{2}{*}{$\begin{array}{l}\text { Wavenumber } \\
\mathrm{cm}^{-1}\end{array}$} & \multicolumn{2}{|c|}{ Intensity } & \multirow{2}{*}{$\begin{array}{l}\text { Energy levels } \\
\mathrm{cm}^{-1}\end{array}$} \\
\hline & & Arc & Spark & \\
\hline $\begin{array}{l}3426.414 \\
3427.853 \\
3434.701 \\
3435.213 \\
3435.679\end{array}$ & $\begin{array}{l}29176.66 \\
29164.41 \\
29106.27 \\
29101.93 \\
29097.98\end{array}$ & $\begin{array}{r}3 \\
60 \\
200 \\
20 \\
40\end{array}$ & $\begin{array}{l}4 \\
1\end{array}$ & $\begin{array}{r}20639_{5_{1 / 2}}-49815^{o}{ }_{4_{1 / 2}} \\
4178_{0_{1 / 2}}-33343^{\circ} o_{1_{1 / 2}} \\
15770_{1_{1 / 2}}-44876^{o}{ }_{1 / / 2} \\
15770_{1_{1 / 2}}-44872^{o}{ }_{2_{1 / 2}} \\
15624_{2_{1 / 2}}-44722^{o}{ }_{1_{1 / 2}}\end{array}$ \\
\hline $\begin{array}{l}3436.644 \\
3437.439 \\
3438.729 \\
3439.217 \\
3439.819\end{array}$ & $\begin{array}{l}29089.81 \\
29083.08 \\
29072.18 \\
29068.05 \\
29062.96\end{array}$ & $\begin{array}{r}3 \\
150 \\
80 \\
3 \\
12\end{array}$ & $\begin{array}{l}1 \\
1\end{array}$ & $\begin{array}{r}19791_{4_{1 / 2}}-48881^{o}{ }_{4_{1 / 2}} \\
4002_{1_{1 / 2}}-33085^{o}{ }_{2_{1 / 2}} \\
14733_{4_{1 / 2}}-43805^{o}{ }_{3_{1 / 2}} \\
17328_{4_{1 / 2}}-46396^{o}{ }_{3_{1 / 2}} \\
15624_{2_{1 / 2}}-44687^{\circ}{ }_{3_{1 / 2}}\end{array}$ \\
\hline $\begin{array}{l}3441.217 \\
3442.337 \\
3443.472 \\
3449.897 \\
3451.050\end{array}$ & $\begin{array}{l}29051.16 \\
29041.70 \\
29032.13 \\
28978.07 \\
28968.38\end{array}$ & $\begin{array}{l}3 w \\
10 \\
200 c \\
15 \\
200\end{array}$ & $\begin{array}{l}2 \\
8\end{array}$ & $\begin{array}{r}10516_{3_{1 / 2}}-39567^{\circ} 3_{1 / 2} \\
13252_{2_{1 / 2}}-42294^{\circ}{ }_{2_{1 / 2}} \\
2572_{4_{1 / 2}}-31605^{\circ}{ }_{3_{1 / 2}} \\
19903_{3_{1 / 2}}-48881^{\circ}{ }_{4_{1 / 2}} \\
15298_{3_{1 / 2}}-44266^{\circ}{ }_{3_{1 / 2}}\end{array}$ \\
\hline $\begin{array}{l}3451.507 \\
3453.020 \\
3454.073 \\
3454.623 \\
3456.849\end{array}$ & $\begin{array}{l}28964.55 \\
28951.86 \\
28943.03 \\
28938.42 \\
28919.79\end{array}$ & $\begin{array}{r}3 \\
30 \\
30 \\
8 \\
200\end{array}$ & $\begin{array}{l}1 \\
1 \\
5\end{array}$ & $\begin{array}{r}13252_{2_{1 / 2}}-42217^{\circ}{ }_{1_{1 / 2}} \\
15770_{1_{1 / 2}}-44722^{\circ}{ }_{1_{1 / 2}} \\
15298_{3_{1 / 2}}-44241^{o} o_{2_{1 / 2}} \\
11890_{0_{1 / 2}}-40829^{\circ} o_{0_{1 / 2}} \\
3700_{2_{1 / 2}}-32620^{\circ}{ }_{3_{1 / 2}}\end{array}$ \\
\hline $\begin{array}{l}3457.243 \\
3457.603 \\
3465.212 \\
3466.278 \\
3470.188\end{array}$ & $\begin{array}{l}28916.49 \\
28913.48 \\
28850.00 \\
28841.12 \\
28808.63\end{array}$ & $\begin{array}{c}400 \\
40 \\
5 \\
5000 \mathrm{cw} \\
10\end{array}$ & $\begin{array}{r}20 \\
3 \\
500 \mathrm{cw}\end{array}$ & $\begin{array}{r}14733_{4_{1 / 2}}-43649^{\circ} o_{4_{1 / 2}} \\
15624_{2_{1 / 2}}-44537^{\circ} o_{2_{1 / 2}} \\
13252_{2_{1 / 2}}-42102^{o} o_{1 / 2} \\
2572_{4_{1 / 2}}-31414^{o} o_{1 / 2} \\
15298_{3_{1 / 2}}-44107^{\circ} o_{2_{1 / 2}}\end{array}$ \\
\hline $\begin{array}{l}3470.299 \\
3472.165 \\
3472.896 \\
3473.726 \\
3475.178\end{array}$ & $\begin{array}{l}28807.71 \\
28792.23 \\
28786.17 \\
28779.29 \\
28767.26\end{array}$ & $\begin{array}{r}5 \\
30 \\
15 \\
20 \\
80\end{array}$ & 2 & $\begin{array}{l}226153_{1 / 2}-51423^{o} 3_{1 / 2} \\
152983_{3_{1 / 2}}-44090^{\circ} 3_{1 / 2} \\
11063_{2_{1 / 2}}-39849^{\circ} 3_{3_{1 / 2}} \\
14733_{4_{1 / 2}}-43512^{\circ} o_{5_{1 / 2}} \\
15770_{1_{1 / 2}}-44537^{\circ} o_{2_{1 / 2}}\end{array}$ \\
\hline $\begin{array}{l}3475.592 \\
3477.838 \\
3478.432 \\
3479.452 \\
3482.288\end{array}$ & $\begin{array}{l}28763.84 \\
28745.26 \\
28740.35 \\
28731.93 \\
28708.53\end{array}$ & $\begin{array}{c}1000 \\
1 \\
10 \\
10 \\
2 h\end{array}$ & 10 & $\begin{array}{r}3250_{3_{1 / 2}}-32014^{o}{ }_{2_{1 / 2}} \\
203843_{3_{1 / 2}}-49130^{o} o_{3_{1 / 2}} \\
13252_{2_{1 / 2}}-41993^{o} o_{3_{1 / 2}} \\
14169_{1_{1 / 2}}-42901^{o} o_{1 / 2} \\
15298_{3_{1 / 2}}-44007_{2_{1 / 2}}^{o}\end{array}$ \\
\hline $\begin{array}{l}3484.620 \\
3486.226 \\
3490.299 \\
3493.394 \\
3494.168\end{array}$ & $\begin{array}{l}28689.32 \\
28676.10 \\
28642.64 \\
28617.26 \\
28610.92\end{array}$ & $\begin{array}{c}60 \\
1000 c \\
100 \\
400 \\
20\end{array}$ & $\begin{array}{c}1 \\
30 c \\
2 \\
5 \\
1\end{array}$ & $\begin{array}{r}156244_{2_{1 / 2}}-44313^{o}{ }_{1_{1 / 2}} \\
3250_{3_{1 / 2}}-31927^{\circ}{ }_{2_{1 / 2}} \\
156244_{2_{1 / 2}}-44266^{\circ} o_{3_{1 / 2}} \\
156242_{2_{1 / 2}}-44241_{2_{1 / 2}}^{o} \\
21204_{4_{1 / 2}}-49815^{o}{ }_{4_{1 / 2}}\end{array}$ \\
\hline $\begin{array}{l}3494.623 \\
3500.161 \\
3500.704 \\
3501.241 \\
3501.507\end{array}$ & $\begin{array}{l}28607.20 \\
28561.94 \\
28557.51 \\
28553.13 \\
28550.96\end{array}$ & $\begin{array}{r}500 \\
6 \\
1000 \\
200 \\
20\end{array}$ & $\begin{array}{c}4 \\
20 \\
2 \\
10 h\end{array}$ & $\begin{array}{r}15298_{3_{1 / 2}}-43905^{o}{ }_{3_{1 / 2}} \\
17146_{5_{1 / 2}}-45708^{\circ}{ }_{4_{1 / 2}} \\
3700_{2_{1 / 2}}-32258^{o} o_{1_{1 / 2}} \\
3700_{2_{1 / 2}}-32253^{o}{ }_{1 / 2} \\
16025_{5_{1 / 2}}-44576^{o}{ }_{4_{1 / 2}}\end{array}$ \\
\hline $\begin{array}{l}3502.467 \\
3502.704 \\
3503.336 \\
3503.586 \\
3503.786\end{array}$ & $\begin{array}{l}28543.13 \\
28541.20 \\
28536.05 \\
28534.02 \\
28532.39\end{array}$ & $\begin{array}{c}80 \\
800 c \\
30 \\
30 \\
40\end{array}$ & $\begin{array}{l}2 \\
20 c \\
20 h w\end{array}$ & $\begin{array}{r}15770{ }_{1_{1 / 2}}-44313^{o}{ }_{1_{1 / 2}} \\
25722_{4_{1 / 2}}-31114^{o}{ }_{4_{1 / 2}} \\
132522_{2_{1 / 2}}-41788^{\circ}{ }_{{ }_{1 / 2}} \\
14733{ }_{4_{1 / 2}}-43267^{\circ}{ }_{3_{1 / 2}} \\
14733{ }_{4_{1 / 2}}-43265^{\circ}{ }_{4_{1 / 2}}\end{array}$ \\
\hline
\end{tabular}


TABLE 3. Classified lines of $\mathrm{TC}_{\mathrm{I}}$

\begin{tabular}{|c|c|c|c|c|}
\hline \multirow{2}{*}{$\begin{array}{c}\text { Wavelength } \\
\text { A }\end{array}$} & \multirow{2}{*}{$\begin{array}{l}\text { Wavenumber } \\
\mathrm{cm}^{-1}\end{array}$} & \multicolumn{2}{|c|}{ Intensity } & \multirow{2}{*}{$\begin{array}{c}\text { Energy levels } \\
\mathrm{cm}^{-1}\end{array}$} \\
\hline & & Arc & Spark & \\
\hline 3505.558 & 28517.97 & 3 & & $11578_{1_{12}}-40096^{o}{ }_{1_{1 / 2}}$ \\
\hline 3506.114 & 28513.44 & 50 & 1 & $11063_{2_{1 / 2}}^{1 / 2}-39576^{o}{ }_{1_{1 / 2}}^{1 / 2}$ \\
\hline 3506.931 & 28506.80 & 10 & & $15298_{3_{1 / 2}}-43805^{0} 0_{1 / 2}^{1 / 2}$ \\
\hline 3507.187 & 28504.72 & $100 c$ & 1 & $11063_{2_{1 / 2}}-39567^{\circ} 0_{3_{1 / 2}}$ \\
\hline 3508.271 & 28495.91 & 100 & 1 & $19791_{4_{1 / 2}}-48287^{\circ} 0_{5_{1 / 2}}$ \\
\hline 3509.519 & 28485.78 & 4 & & $15624_{2_{1 / 2}}-44110^{o}{ }_{1_{1 / 2}}$ \\
\hline 3509.869 & 28482.94 & 2 & & $15624_{2_{1 / 2}}^{1 / 2}-44107_{2_{1 / 2}}^{1 / 2}$ \\
\hline 3510.594 & 28477.06 & 10 & & $13252_{2_{1 / 2}}^{1 / 2}-41729^{\circ}{ }_{3_{1 / 2}}$ \\
\hline 3511.328 & 28471.11 & 20 & & $15770_{1_{1 / 2}}-44241^{\circ}{ }_{2_{1 / 2}}$ \\
\hline 3511.919 & 28466.31 & 20 & 1 & $15624_{2_{1 / 2}}-44090^{0} o_{3_{1 / 2}}^{1 / 2}$ \\
\hline 3512.546 & 28461.23 & 5 & & $16415_{2_{1 / 2}}-44876_{1_{1 / 2}}^{o}$ \\
\hline 3512.768 & 28459.44 & 6 & & $20639_{5_{1 / 2}}-49098^{\circ}{ }_{4_{1 / 2}^{1 / 2}}$ \\
\hline 3514.700 & 28443.79 & 2 & & $20686_{2_{1 / 2}}-49130^{\circ}{ }_{3_{1 / 2}}$ \\
\hline 3515.174 & 28439.96 & 12 & 1 & $19702_{1_{1 / 2}}-48142_{2_{1 / 2}}^{o}$ \\
\hline 3517.206 & 28423.53 & 40 & 1 & $16415_{2_{1 / 2}}^{1 / 2}-44839^{\circ} 0_{3_{1 / 2}}$ \\
\hline 3517.442 & 28421.62 & 10 & & $19791_{4_{1 / 2}}-48213_{4_{1 / 2}}^{o}$ \\
\hline 3520.054 & 28400.53 & $30 c$ & & $4002_{1_{1 / 2}}^{1 / 2}-32403^{o} 0_{1 / 2}^{1 / 2}$ \\
\hline 3520.276 & 28398.74 & 20 & & $11578_{1_{1 / 2}}-39977^{\circ} 0_{1 / 2}$ \\
\hline 3520.514 & 28396.82 & $30 c$ & & $11063_{2_{1 / 2}}^{1 / 2}-39459^{\circ}{ }_{2_{1 / 2}}^{1 / 2}$ \\
\hline 3522.275 & 28382.62 & 30 & & $15624_{2_{1 / 2}}-44007^{0}{ }_{2_{1 / 2}}$ \\
\hline 3522.516 & 28380.68 & 15 & & $17328_{4_{1 / 2}}-45708^{o}{ }_{4_{1 / 2}}$ \\
\hline 3524.523 & 28364.52 & 20 & 1 & $14733_{4_{1 / 2}}-43097^{\circ}{ }_{4 / 2}$ \\
\hline 3525.828 & 28354.02 & 800 & 15 & $3250_{3_{1 / 2}}-31605^{\circ}{ }_{3_{1 / 2}}$ \\
\hline 3526.178 & 28351.21 & 300 & 5 & $15298_{3_{1 / 2}}-43649^{\circ}{ }_{4_{1 / 2}}$ \\
\hline 3527.626 & 28339.57 & 50 & 2 & $15770_{1_{1 / 2}}-44110_{1_{1 / 2}}^{4_{1 / 2}}$ \\
\hline 3527.976 & 28336.76 & 30 & & $15770_{1_{1 / 2}}-44107_{2_{1 / 2}}^{o}$ \\
\hline 3529.829 & 28321.88 & 100 & 2 & $19895_{2_{1 / 2}}^{1 / 2}-48217^{0} 3_{1 / 2}^{2_{1 / 2}}$ \\
\hline 3530.813 & 28313.99 & 10 & & $3700_{2}^{1 / 2}-32014^{o} o_{1 / 2}$ \\
\hline 3531.002 & 28312.480 & 3 & & $11578_{1_{1 / 2}}^{1 / 2}-39891_{2_{1 / 2}}^{1 / 2}$ \\
\hline 3531.318 & 28309.94 & 15 & & $19903_{3_{1 / 2}}-48213_{4_{1 / 2}}^{o}$ \\
\hline 3531.733 & 28306.62 & 3 & & $16415_{2_{1 / 2}}-44722^{o}{ }_{1_{1 / 2}}$ \\
\hline 3534.878 & 28281.43 & 150 & 3 & $15624_{2_{1 / 2}}-43905^{\circ} o_{1 / 2}$ \\
\hline 3535.506 & 28276.41 & 500 & 12 & $13252_{2_{1 / 2}}^{1 / 2}-41529^{\circ} o_{3_{1 / 2}}$ \\
\hline 3538.119 & 28255.53 & 300 & & $4002_{1_{1 / 2}}-32258^{\circ}{ }_{1 / 2}$ \\
\hline 3538.678 & 28251.06 & 800 & 3 & $4002_{1_{1 / 2}}-32253_{1_{1 / 2}}^{o}$ \\
\hline 3539.148 & 28247.31 & 20 & & $19895_{2_{1 / 2}}-48142_{2_{1 / 2}}^{o}$ \\
\hline 3539.759 & 28242.44 & 12 & & $20639_{5_{1 / 2}}^{1 / 2}-48881_{4_{1 / 2}}^{0}$ \\
\hline 3540.174 & 28239.12 & 1 & & $19903_{3_{1 / 2}}-48142_{2_{1 / 2}}^{o}$ \\
\hline 3541.772 & 28226.38 & $2000 c$ & $20 c$ & $3700_{2_{1 / 2}}-31927^{\circ} 2_{1 / 2}^{1 / 2}$ \\
\hline 3544.382 & 28205.60 & 20 & 1 & $11890_{0_{1 / 2}}^{1_{1 / 2}}-40096^{o}{ }_{1 / 2}^{1 / 2}$ \\
\hline 3549.725 & 28163.15 & $6000 c$ & $60 c$ & $3250_{3_{1 / 2}}-31414_{3_{1 / 2}}^{o}$ \\
\hline 3550.645 & 28155.85 & $4000 c$ & $40 c$ & $3250_{3_{1 / 2}}-31406_{2_{1 / 2}}^{o}$ \\
\hline 3551.206 & 28151.40 & 30 & & $0_{2_{1 / 2}}-28151_{1_{1 / 2}}^{o / / 2}$ \\
\hline 3553.539 & 28132.92 & 60 & 1 & $16133_{4_{1 / 2}}^{1 / 2}-44266^{o} o_{1 / 2}^{1 / 2}$ \\
\hline 3554.580 & 28124.68 & 30 & & $14169_{1_{1 / 2}}^{1 / 2}-42294^{o} o_{1 / 2}^{1 / 2}$ \\
\hline 3554.919 & 28122.00 & 2 & & $16415_{2_{1 / 2}}-44537^{\circ}{ }_{2_{1 / 2}}$ \\
\hline 3556.056 & 28113.01 & 40 & $2 h$ & $16025_{5_{1 / 2}}^{1 / 2}-44138_{5_{1 / 2}}^{o}$ \\
\hline 3558.209 & 28096.00 & 2 & & $21719_{5_{1 / 2}}-49815^{\circ}{ }_{4_{1 / 2}}$ \\
\hline 3559.754 & 28083.80 & 300 & 5 & $14733_{4_{1 / 2}}^{1 / 2}-42817^{0}{ }_{1 / 2}^{4 / 2}$ \\
\hline 3560.319 & 28079.35 & 800 & 15 & $4178_{0_{1 / 2}}-32258^{o}{ }_{1_{1 / 2}}^{4}$ \\
\hline
\end{tabular}


TABLE 3. Classified lines of Tc I

\begin{tabular}{|c|c|c|c|c|}
\hline \multirow{2}{*}{$\begin{array}{c}\text { Wavelength } \\
\text { A }\end{array}$} & \multirow{2}{*}{$\begin{array}{l}\text { Wavenumber } \\
\mathrm{cm}^{-1}\end{array}$} & \multicolumn{2}{|c|}{ Intensity } & \multirow{2}{*}{$\begin{array}{l}\text { Energy levels } \\
\mathrm{cm}^{-1}\end{array}$} \\
\hline & & Arc & Spark & \\
\hline 3560.876 & 28074.95 & 50 & & $4178_{0_{1 / 2}}-32253^{o}{ }_{1_{1 / 2}}$ \\
\hline 3561.192 & 28072.46 & 50 & 1 & $19791_{4_{1 / 2}}-47864^{o}{ }_{3_{1 / 2}}^{1 / 2}$ \\
\hline 3562.303 & 28063.71 & 4 & & $21752_{3_{1 / 2}}-49815^{\circ}{ }_{4_{1 / 2}}$ \\
\hline 3564.359 & 28047.52 & 5 & & $14169_{1_{1 / 2}}-42217^{\circ}{ }_{1_{1 / 2}}$ \\
\hline 3565.217 & 28040.77 & 100 & 1 & $14733_{4_{1 / 2}}-42773_{5_{1 / 2}}^{o}$ \\
\hline 3565.490 & 28038.62 & 80 & 1 & $14733_{4_{1 / 2}}-42771_{3_{1 / 2}}^{o}$ \\
\hline 3566.883 & 28027.67 & 3 & & $19791_{4_{1 / 2}}-47819^{\circ}{ }_{4_{1 / 2} / 2}$ \\
\hline 3568.853 & 28012.20 & 800 & 5 & $4002_{1_{1 / 2}}-32014^{o / 2} o_{2_{1 / 2}}^{T / 2}$ \\
\hline 3570.654 & 27998.08 & 100 & 1 & $11578_{1_{1 / 2}}-39576_{1_{1 / 2}}^{0}$ \\
\hline 3570.654 & 27998.08 & 100 & 1 & $13252_{2_{1 / 2}}-41250^{\circ}{ }_{1_{1 / 2}}$ \\
\hline 3573.083 & 27979.04 & 50 & & $16287_{31,2}-44266_{3_{1 / 2}}^{o}$ \\
\hline 3574.410 & 27968.66 & 25 & & $15298_{3_{1 / 2}}-43267_{3_{1 / 2}}$ \\
\hline 3574.610 & 27967.09 & 80 & 1 & $15298_{3_{1 / 2}}-43265^{\circ}{ }_{4_{1 / 2}}$ \\
\hline 3575.420 & 27960.76 & 100 & 1 & $19903_{3_{1 / 2}}-47864^{o} o_{3_{1 / 2}}$ \\
\hline 3575.964 & 27956.50 & 20 & & $16133_{4_{1 / 2}}-44090^{\circ}{ }_{3_{1 / 2}}$ \\
\hline 3576.324 & 27953.69 & 50 & & $16287_{3_{1 / 2}}-44241_{2_{1 / 2}}^{o}$ \\
\hline 3577.228 & 27946.62 & 6 & & $16025_{5_{1 / 2}}-43971_{4_{1 / 2}}^{0}$ \\
\hline 3577.986 & 27940.70 & 15 & & $0_{2_{1 / 2}}-27940^{\circ}{ }_{2_{1 / 2}}$ \\
\hline 3578.969 & 27933.03 & 60 & & $14169_{1_{1 / 2}^{1 / 2}}^{2}-42102_{2_{1 / 2}}^{o}$ \\
\hline 3580.059 & 27924.52 & 1000 & $10 c$ & $4002_{1_{1 / 2}}-31927_{2_{1 / 2}}^{-}$ \\
\hline 3581.258 & 27915.18 & 1000 & $10 c$ & $16428_{2_{1 / 2}}-44343_{3_{1 / 2}}$ \\
\hline 3581.744 & 27911.39 & 10 & & $20231_{1_{1 / 2}}-48142_{2_{1 / 2}}^{o}$ \\
\hline 3582.075 & 27908.81 & 800 & 4 & $16428_{2_{1 / 2}}-44337_{2_{1 / 2}}^{1 / 2}$ \\
\hline 3582.628 & 27904.50 & 2000 & 10 & $3700_{2_{1 / 2}}-31605^{o} o_{3_{1 / 2}}$ \\
\hline 3582.628 & 27904.50 & 2000 & 10 & $16428_{2_{1 / 2}}^{o}-44333_{1_{1 / 2}}$ \\
\hline 3583.464 & 27897.99 & 12 & & $16415_{2_{1 / 2}}-44313_{1_{1 / 2}}^{o}$ \\
\hline 3584.005 & 27893.78 & 1 & & $21204_{4_{1 / 2}}-49098^{\circ}{ }_{4_{1 / 2}}$ \\
\hline 3585.684 & 27880.72 & 30 & 1 & $14733_{4_{1 / 2}}-42613^{o}{ }_{4_{1 / 2}}$ \\
\hline 3587.386 & 27867.49 & 1 & & $14733_{4_{1 / 2}}-42600_{5_{1 / 2}}^{o}$ \\
\hline 3587.942 & 27863.17 & 4000 & 40 & $3250_{3_{1 / 2}}-31114_{4_{1 / 2}}^{\circ}$ \\
\hline 3589.471 & 27851.31 & 15 & & $16415_{2_{1 / 2}}-44266_{3_{1 / 2}}^{0}$ \\
\hline 3591.222 & 27837.73 & 50 & 1 & $16133_{4_{1 / 2}}-43971_{4_{1 / 2}}^{\circ}$ \\
\hline 3591.483 & 27835.70 & 80 & 4 & $13252_{2_{1 / 2}}-41088^{\circ}{ }_{3_{1 / 2}}^{1 / 2}$ \\
\hline 3591.942 & 27832.15 & $8 c w$ & & $16025_{5_{1 / 2}}-43857_{6_{1 / 2}}^{0}$ \\
\hline 3593.580 & 27819.46 & 10 & & $16287_{3_{1 / 2}}-44107_{2_{1 / 2}}^{0}$ \\
\hline 3594.573 & 27811.78 & 300 & 4 & $14733_{4_{1 / 2}}-42545^{0}{ }_{4_{1 / 2}}$ \\
\hline 3594.908 & 27809.18 & $50 \mathrm{cw}$ & & $2572_{4_{1 / 2}}-30382_{3_{1 / 2}}^{o}$ \\
\hline 3595.661 & 27803.36 & $1000 \mathrm{cw}$ & $10 \mathrm{cw}$ & $3700_{2_{1 / 2}}-31503^{o}{ }_{1_{1 / 2}}$ \\
\hline 3596.181 & 27799.34 & 4 & & $15298_{3_{1 / 2}}-43097^{\circ}{ }_{4_{1 / 2}}$ \\
\hline 3597.401 & 27789.91 & 12 & & $14733_{4_{1 / 2}}-42523_{3_{1 / 2}}^{0}$ \\
\hline 3598.830 & 27778.88 & 30 & & $15298_{3_{1 / 2}}-43077_{3_{1 / 2}}^{0}$ \\
\hline 3599.750 & 27771.78 & 50 & 1 & $16133_{4_{1 / 2}}-43905_{3_{1 / 2}}^{0}$ \\
\hline 3602.191 & 27752.96 & 20 & 1 & $14169_{1_{1 / 2}}-41922^{\circ}{ }_{2_{1 / 2}}$ \\
\hline 3602.639 & 27749.51 & 1 & & $19702_{1_{1 / 2}}-47452_{2_{1 / 2}}^{o}$ \\
\hline 3605.938 & 27724.12 & 15 & & $10516_{3_{1 / 2}}-38240^{\circ}{ }_{3_{1 / 2}}$ \\
\hline 3607.321 & 27713.49 & $1000 c$ & $30 c$ & $3700_{2_{1 / 2}}-31414_{3_{1 / 2}}^{0}$ \\
\hline 3607.625 & 27711.16 & 200 & 5 & $14733_{4_{1 / 2}}-42444_{5_{1 / 2}}^{0}$ \\
\hline 3608.271 & 27706.20 & $2000 c$ & $80 c$ & $3700_{2_{1 / 2}}-31406_{2_{1 / 2}}^{0}$ \\
\hline 3609.045 & 27700.26 & 60 & & $10516_{3_{1 / 2}}^{-1 / 2}-38216_{2_{1 / 2}}^{0}$ \\
\hline 3609.843 & 27694.13 & & & $20523_{2_{1 / 2}}-48217^{\circ}{ }_{3_{1 / 2}}$ \\
\hline
\end{tabular}


TABLE 3. Classified lines of $\mathrm{TC} \mathrm{I}$

\begin{tabular}{|c|c|c|c|c|}
\hline \multirow{2}{*}{$\begin{array}{c}\text { Wavelength } \\
\text { A }\end{array}$} & \multirow{2}{*}{$\begin{array}{l}\text { Wavenumber } \\
\mathrm{cm}^{-1}\end{array}$} & \multicolumn{2}{|c|}{ Intensity } & \multirow{2}{*}{$\begin{array}{l}\text { Energy levels } \\
\mathrm{cm}^{-1}\end{array}$} \\
\hline & & Arc & Spark & \\
\hline $\begin{array}{l}3611.156 \\
3612.108 \\
3612.346 \\
3612.833 \\
3614.282\end{array}$ & $\begin{array}{l}27684.06 \\
27676.77 \\
27674.94 \\
27671.21 \\
27660.12\end{array}$ & $\begin{array}{r}50 \\
60 \\
3 \\
4 \\
15\end{array}$ & $3 h$ & $\begin{array}{r}162873_{3_{1 / 2}}-43971^{0}{ }_{4_{1 / 2}} \\
212044_{4_{1 / 2}}-48881^{0}{ }_{4_{1 / 2}} \\
164155_{2_{1 / 2}}-44090^{\circ}{ }_{3_{1 / 2}} \\
161333_{4_{1 / 2}}-43805^{0}{ }_{3_{1 / 2}} \\
0_{2_{1 / 2}}-27660^{o} o_{3_{1 / 2}}\end{array}$ \\
\hline $\begin{array}{l}3615.814 \\
3616.528 \\
3618.943 \\
3619.664 \\
3622.453\end{array}$ & $\begin{array}{l}27648.40 \\
27642.94 \\
27624.50 \\
27618.99 \\
27597.73\end{array}$ & $\begin{array}{r}50 \\
30 \\
200 \\
20 \\
1\end{array}$ & $\begin{array}{l}1 \\
3\end{array}$ & $\begin{array}{l}206399_{5_{1 / 2}}-48287^{0}{ }_{5_{1 / 2}} \\
15624{ }_{2_{1 / 2}}-43267^{\circ} 0^{0} 3_{1 / 2} \\
160255_{5_{1 / 2}}-43649^{\circ}{ }_{4_{1 / 2}} \\
141699_{1_{1 / 2}}-41788^{\circ}{ }_{1_{1 / 2}} \\
19791_{4_{1 / 2}}-47389^{0} o_{3_{1 / 2}}\end{array}$ \\
\hline $\begin{array}{l}3623.508 \\
3625.555 \\
3626.294 \\
3627.364 \\
3627.858\end{array}$ & $\begin{array}{l}27589.69 \\
27574.12 \\
27568.50 \\
27560.37 \\
27556.61\end{array}$ & $\begin{array}{r}5 \\
150 \\
10 \\
1000 c \\
100\end{array}$ & $\begin{array}{c}3 \\
10 c \\
2\end{array}$ & $\begin{array}{r}172823_{3_{1 / 2}}-44872^{o}{ }_{2_{1 / 2}} \\
206399_{5_{1 / 2}}-48213^{o}{ }_{4_{1 / 2}} \\
132522_{2_{1 / 2}}-40821^{o}{ }_{2_{1 / 2}} \\
25722_{4_{1 / 2}}-30133^{o}{ }_{{ }_{1 / 2}} \\
172822_{3_{1 / 2}}-44839^{o}{ }_{3_{1 / 2}}\end{array}$ \\
\hline $\begin{array}{l}3628.476 \\
3628.901 \\
3630.388 \\
3631.227 \\
3633.244\end{array}$ & $\begin{array}{l}27551.92 \\
27548.69 \\
27537.41 \\
27531.05 \\
27515.76\end{array}$ & $\begin{array}{r}2 \\
30 \\
200 \\
2 \\
150\end{array}$ & $\begin{array}{l}1 \\
2 \\
2\end{array}$ & $\begin{array}{l}19791_{4_{1 / 2}}-47343^{o}{ }_{4_{1 / 2}} \\
199033_{3_{1 / 2}}-47452^{o}{ }_{2_{1 / 2}} \\
14679_{0_{1 / 2}}-42217^{0}{ }_{1_{1 / 2}} \\
20686_{2_{1 / 2}}-48217^{0} o_{3_{1 / 2}} \\
16133_{4_{1 / 2}}-43649^{o}{ }_{4_{1 / 2}}\end{array}$ \\
\hline $\begin{array}{l}3635.146 \\
3636.070 \\
3637.447 \\
3638.012 \\
3638.220\end{array}$ & $\begin{array}{l}27501.37 \\
27494.38 \\
27483.97 \\
27479.70 \\
27478.13\end{array}$ & $\begin{array}{c}3000 c \\
10000 c \\
8 \\
1 \\
1000\end{array}$ & $\begin{array}{r}50 c \\
500 c\end{array}$ & 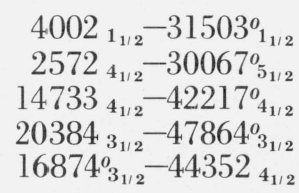 \\
\hline $\begin{array}{l}3638.848 \\
3639.379 \\
3640.226 \\
3641.093 \\
3641.183\end{array}$ & $\begin{array}{l}27473.39 \\
27469.38 \\
27462.99 \\
27456.45 \\
27455.77\end{array}$ & $\begin{array}{r}200 \\
900 \\
400 \\
6 \\
15\end{array}$ & $\begin{array}{r}4 \\
15 \\
10 \\
1 \\
1\end{array}$ & $\begin{array}{l}152988_{3_{1 / 2}}-42771^{o}{ }_{3_{1 / 2}} \\
168744^{o}{ }_{3_{1 / 2}}-443433^{3_{1 / 2}} \\
168744^{o}{ }_{3_{1 / 2}}-44337^{2_{1 / 2}} \\
20686_{2_{1 / 2}}-48142^{o}{ }_{2_{1 / 2}} \\
14169_{1_{1 / 2}}-41625^{\circ}{ }_{1_{1 / 2}}\end{array}$ \\
\hline $\begin{array}{l}3641.534 \\
3643.376 \\
3643.950 \\
3644.134 \\
3644.427\end{array}$ & $\begin{array}{l}27453.13 \\
27439.25 \\
27434.92 \\
27433.54 \\
27431.33\end{array}$ & $\begin{array}{r}30 \\
10 \\
8 \\
50 \\
3\end{array}$ & 1 & $\begin{array}{l}156244_{2_{1 / 2}}-43077^{\circ} o_{3_{1 / 2}} \\
20231_{1_{1 / 2}}-47670^{o} o_{0_{1 / 2}} \\
203844_{3_{1 / 2}}-47819^{\circ}{ }_{a_{1 / 2}} \\
207833_{3_{1 / 2}}-48217^{\circ}{ }_{3_{1 / 2}} \\
214500_{4_{1 / 2}}-48881^{o} o_{4_{1 / 2}}\end{array}$ \\
\hline $\begin{array}{l}3648.040 \\
3648.860 \\
3650.344 \\
3651.467 \\
3654.054\end{array}$ & $\begin{array}{l}27404.17 \\
27398.01 \\
27386.87 \\
27378.45 \\
27359.07\end{array}$ & $\begin{array}{c}1000 c \\
10 \\
60 \\
600 \\
10\end{array}$ & $\begin{array}{r}50 c \\
2 \\
20 \\
1\end{array}$ & $\begin{array}{r}4002_{1_{1 / 2}}-31406^{o}{ }_{2_{1 / 2}} \\
152988_{3_{1 / 2}}-42696^{o} o_{2_{1 / 2}} \\
15624_{2_{1 / 2}}-43011^{o}{ }_{2_{1 / 2}} \\
161333_{4_{1 / 2}}-43512^{o}{ }_{5_{1 / 2}} \\
173288_{4_{1 / 2}}-44687^{0} o_{3_{1 / 2}}\end{array}$ \\
\hline $\begin{array}{l}3656.458 \\
3658.588 \\
3659.882 \\
3661.452 \\
3662.032\end{array}$ & $\begin{array}{l}27341.08 \\
27325.16 \\
27315.50 \\
27303.79 \\
27299.46\end{array}$ & $\begin{array}{c}1 \\
1000 c \\
3 \\
400 c \\
6\end{array}$ & $\begin{array}{l}60 c \\
30 c\end{array}$ & $\begin{array}{r}205233_{2_{1 / 2}}-47864^{o}{ }_{3_{1 / 2}} \\
41788_{0_{1 / 2}}-31503^{o}{ }_{1_{1 / 2}} \\
15298_{3_{1 / 2}}-42613^{\circ}{ }_{4_{1 / 2}} \\
165533_{6_{1 / 2}}-43857^{0}{ }_{6_{1 / 2}} \\
14733{ }_{4_{1 / 2}}-42032^{o} o_{5_{1 / 2}}\end{array}$ \\
\hline $\begin{array}{l}3662.838 \\
3663.091 \\
3664.455 \\
3664.916 \\
3667.349\end{array}$ & $\begin{array}{l}27293.46 \\
27291.57 \\
27281.41 \\
27277.98 \\
27259.88\end{array}$ & $\begin{array}{r}30 \\
8 \\
6 \\
200 \\
10\end{array}$ & 1 & 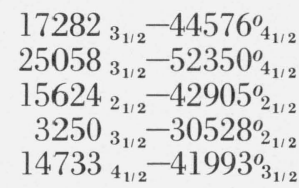 \\
\hline
\end{tabular}


TAB LE 3. Classified lines of $\mathrm{Tc} \mathrm{I}$

\begin{tabular}{|c|c|c|c|c|}
\hline \multirow{2}{*}{$\begin{array}{c}\text { Wavelength } \\
\text { A }\end{array}$} & \multirow{2}{*}{$\underset{\mathrm{cm}^{-1}}{\text { Wavenumber }}$} & \multicolumn{2}{|c|}{ Intensity } & \multirow{2}{*}{$\begin{array}{c}\text { Energy levels } \\
\mathrm{cm}^{-1}\end{array}$} \\
\hline & & Arc & Spark & \\
\hline $\begin{array}{l}3667.510 \\
3667.794 \\
3667.991 \\
3668.940 \\
3669.152\end{array}$ & $\begin{array}{l}27258.69 \\
27256.58 \\
27255.11 \\
27248.06 \\
27246.49\end{array}$ & $\begin{array}{l}30 \\
10 \\
10 \\
20 \\
40\end{array}$ & $\begin{array}{l}1 \\
2\end{array}$ & $\begin{array}{l}16287_{3_{1 / 2}}-43546^{\circ}{ }_{2_{1 / 2}} \\
11063{ }_{2_{1 / 2}}-38319^{\circ}{ }_{1_{1 / 2}} \\
17282_{3_{1 / 2}}-445377^{\circ}{ }_{{ }_{1 / 2}} \\
173288_{4_{1 / 2}}-44576^{\circ}{ }_{4_{1 / 2}} \\
152988_{3_{1 / 2}}-42545^{\circ}{ }_{4_{1 / 2}}\end{array}$ \\
\hline $\begin{array}{l}3669.918 \\
3672.087 \\
3675.003 \\
3675.003 \\
3675.429\end{array}$ & $\begin{array}{l}27240.80 \\
27224.71 \\
27203.11 \\
27203.11 \\
27199.96\end{array}$ & $\begin{array}{r}40 \\
8 \\
3 \\
3 \\
30\end{array}$ & $1 h$ & $\begin{array}{l}15770_{1_{1 / 2}}-43011^{o}{ }_{2_{1 / 2}} \\
15298_{3_{1 / 2}}-42523^{o}{ }_{3_{1 / 2}} \\
19827_{0_{1 / 2}}-47030^{o}{ }_{1_{1 / 2}} \\
21926_{2_{1 / 2}}-49130^{o}{ }_{3_{1 / 2}} \\
22615_{3_{1 / 2}}-49815^{o}{ }_{4_{1 / 2}}\end{array}$ \\
\hline $\begin{array}{l}3678.089 \\
3678.388 \\
3679.146 \\
3680.318 \\
\overline{3} 680.575\end{array}$ & $\begin{array}{l}27180.29 \\
27178.08 \\
27172.48 \\
27163.83 \\
27161.93\end{array}$ & $\begin{array}{r}80 \\
70 \\
1000 \\
300 \\
50\end{array}$ & $\begin{array}{r}2 \\
2 \\
30 \\
6\end{array}$ & $\begin{array}{l}20639_{5_{1 / 2}}-47819^{\circ}{ }_{4_{1 / 2}} \\
20686_{2_{1 / 2}}-47864^{\circ}{ }_{3_{1 / 2}} \\
16025_{5_{1 / 2}}-43197^{\circ}{ }_{6} 6_{1 / 2} \\
14733_{4_{1 / 2}}-41897^{\circ}{ }_{4_{1 / 2}} \\
21719_{5_{1 / 2}}^{-48881^{o}{ }_{4_{1 / 2}}}\end{array}$ \\
\hline $\begin{array}{l}3681.683 \\
3682.592 \\
3684.744 \\
3687.772 \\
3691.324\end{array}$ & $\begin{array}{l}27153.76 \\
27147.05 \\
27131.20 \\
27108.92 \\
27082.84\end{array}$ & $\begin{array}{r}40 \\
100 \\
5000 \\
20 \\
1\end{array}$ & $\begin{array}{r}2 \\
100 \\
1\end{array}$ & $\begin{array}{r}11063_{2_{1 / 2}}-38216^{o} o_{2_{1 / 2}} \\
16025_{5_{1 / 2}}-43172^{o} 5_{1 / 2} \\
3250_{3_{1 / 2}}-30382^{\circ}{ }_{31 / 2} \\
14679_{0_{1 / 2}}-41788^{o}{ }_{1_{1 / 2}} \\
21204_{4_{1 / 2}}-48287^{\circ}{ }_{5_{1 / 2}}\end{array}$ \\
\hline $\begin{array}{l}3692.759 \\
3693.400 \\
3695.734 \\
3696.297 \\
3697.415\end{array}$ & $\begin{array}{l}27072.31 \\
27067.62 \\
27050.52 \\
27046.40 \\
27038.22\end{array}$ & $\begin{array}{r}300 \\
8 \\
1 \\
150 \\
100\end{array}$ & $\begin{array}{l}3 \\
1\end{array}$ & $\begin{array}{l}15624_{2_{1 / 2}}-42696^{o}{ }_{2_{1 / 2}} \\
20384_{3_{1 / 2}}-47452^{\circ} o_{2_{1 / 2}} \\
20231_{1_{1 / 2}}-47281^{o}{ }_{2_{1 / 2}} \\
13252_{2_{1 / 2}}-40299^{\circ} o_{2_{1 / 2}} \\
16133_{4_{1 / 2}}-43172^{\circ} o_{5_{1 / 2}}\end{array}$ \\
\hline $\begin{array}{l}3697.736 \\
3701.478 \\
3701.966 \\
3703.830 \\
3704.797\end{array}$ & $\begin{array}{l}27035.88 \\
27008.54 \\
27004.98 \\
26991.39 \\
26984.35\end{array}$ & $\begin{array}{r}30 \\
5 \\
1 \\
800 \\
300\end{array}$ & $\begin{array}{r}1 \\
1 \\
20 \\
5\end{array}$ & $\begin{array}{l}20783_{3_{1 / 2}}-47819^{\circ}{ }_{4_{1 / 2}} \\
21204_{4_{1 / 2}}-48213^{\circ}{ }_{4_{1 / 2}} \\
20384_{3_{1 / 2}}-47389^{\circ}{ }_{3_{1 / 2}} \\
171466_{5_{1 / 2}}-44138^{o} o_{5_{1 / 2}} \\
17282_{3_{1 / 2}}-44266^{o}{ }_{3_{1 / 2}}\end{array}$ \\
\hline $\begin{array}{l}3705.481 \\
3705.712 \\
3706.705 \\
3707.627 \\
3708.261\end{array}$ & $\begin{array}{l}26979.37 \\
26977.69 \\
26970.46 \\
26963.75 \\
26959.14\end{array}$ & $\begin{array}{r}100 \\
80 \\
200 \\
200 \\
200\end{array}$ & $\begin{array}{l}2 \\
1 \\
4 \\
5 \\
3\end{array}$ & $\begin{array}{l}16287_{3_{1 / 2}}-43267^{\circ} 3_{1 / 2} \\
16287_{3_{1 / 2}}-43265^{\circ}{ }_{4_{1 / 2}} \\
14733_{4_{1 / 2}}-41703^{\circ}{ }_{4_{1 / 2}} \\
16133_{4_{1 / 2}}-43097^{\circ}{ }_{4_{1 / 2}} \\
16553_{6_{1 / 2}}-43512^{\circ} o_{5_{1 / 2}}\end{array}$ \\
\hline $\begin{array}{l}3709.226 \\
3709.527 \\
3709.831 \\
3710.126 \\
3710.428\end{array}$ & $\begin{array}{l}26952.13 \\
26949.94 \\
26947.73 \\
26945.59 \\
26943.40\end{array}$ & $\begin{array}{r}2 \\
15 \\
80 \\
90 \\
4\end{array}$ & $\begin{array}{l}1 \\
2\end{array}$ & $\begin{array}{l}15298_{3_{1 / 2}}-42250^{o}{ }_{2_{1 / 2}} \\
22180_{4_{1 / 2}}-49130^{\circ} 3_{3_{1 / 2}} \\
15298_{3_{1 / 2}}-42246^{\circ}{ }_{3_{1 / 2}} \\
14679_{0_{1 / 2}}-41625^{\circ}{ }_{1_{1 / 2}} \\
16133_{4_{1 / 2}}-43077^{\circ}{ }_{3_{1 / 2}}\end{array}$ \\
\hline $\begin{array}{l}3711.048 \\
3711.388 \\
3712.256 \\
3712.819 \\
3713.838\end{array}$ & $\begin{array}{l}26938.90 \\
26936.43 \\
26930.13 \\
26926.05 \\
26918.66\end{array}$ & $\begin{array}{r}2 \\
80 \\
1000 \\
300 \\
25\end{array}$ & $\begin{array}{r}1 \\
10 \\
3\end{array}$ & $\begin{array}{r}17328_{4_{1 / 2}}-44266^{o}{ }_{3_{1 / 2}} \\
21206_{2_{1 / 2}}-48142^{o}{ }_{2_{1 / 2}} \\
3700_{2_{1 / 2}}-30630^{o}{ }_{1_{1 / 2}} \\
15770_{1_{1 / 2}}-42696^{o}{ }_{2_{1 / 2}} \\
15298_{3_{1 / 2}}-4217^{\circ} o_{4_{1 / 2}}\end{array}$ \\
\hline $\begin{array}{l}3715.944 \\
3716.809 \\
3718.861 \\
3721.056 \\
3723.132\end{array}$ & $\begin{array}{l}26903.41 \\
26897.14 \\
26882.30 \\
26866.45 \\
26851.47\end{array}$ & $\begin{array}{r}500 \\
20 \\
10000 \\
3 \\
40\end{array}$ & $\begin{array}{r}5 \\
2 \\
300\end{array}$ & $\begin{array}{r}14169_{1_{1 / 2}}-41073^{o}{ }_{1_{1 / 2}} \\
20384_{3_{1 / 2}}-47281^{o}{ }_{2_{1 / 2}} \\
3250_{3_{1 / 2}}-30133^{o}{ }_{4_{1 / 2}} \\
20523_{2_{1 / 2}}-47389^{\circ} o_{3_{1 / 2}} \\
16415_{2_{1 / 2}}-43267^{\circ}{ }_{3_{1 / 2}}\end{array}$ \\
\hline
\end{tabular}


TABLE 3. Classified lines of Tc I

\begin{tabular}{|c|c|c|c|c|}
\hline \multirow{2}{*}{$\begin{array}{c}\text { Wavelength } \\
\text { A }\end{array}$} & \multirow{2}{*}{$\begin{array}{l}\text { Wavenumber } \\
\mathrm{cm}^{-1}\end{array}$} & \multicolumn{2}{|c|}{ Intensity } & \multirow{2}{*}{$\underset{\substack{\text { Energy levels } \\
\mathrm{cm}^{-1}}}{ }$} \\
\hline & & Arc & Spark & \\
\hline $\begin{array}{l}3723.674 \\
3724.176 \\
3724.395 \\
3725.082 \\
3726.152\end{array}$ & $\begin{array}{l}26847.56 \\
26843.94 \\
26842.36 \\
26837.41 \\
26829.70\end{array}$ & $\begin{array}{r}1500 \\
10 \\
2000 \\
20 \\
100\end{array}$ & $\begin{array}{r}15 \\
20 \\
2\end{array}$ & $\begin{array}{l}14733_{4_{1 / 2}}-41580^{o}{ }_{5_{1 / 2}} \\
13252_{2_{1 / 2}}-40096^{o}{ }_{1_{1 / 2}} \\
17522^{o}{ }_{{ }_{1 / 2}}-44365_{5_{1 / 2}} \\
214550_{4_{1 / 2}}-48287^{0} o_{5_{1 / 2}} \\
17522^{o}{ }_{4_{1 / 2}}-44352_{4_{1 / 2}}\end{array}$ \\
\hline $\begin{array}{l}3726.351 \\
3727.364 \\
3728.886 \\
3728.886 \\
3729.177\end{array}$ & $\begin{array}{l}26828.27 \\
26820.98 \\
26810.03 \\
26810.03 \\
26807.94\end{array}$ & $\begin{array}{r}5000 \\
200 \\
30 \\
30 \\
400 c\end{array}$ & $5 c$ & $\begin{array}{c}3700_{2_{1 / 2}}-30528^{o}{ }_{2_{1 / 2}} \\
17522^{o}{ }_{4_{1 / 2}}-443433_{3_{1 / 2}} \\
16287_{3_{1 / 2}}-43097^{\circ}{ }_{4_{1 / 2}} \\
173288_{4_{1 / 2}}-44138^{o}{ }_{5_{1 / 2}} \\
17282_{3_{1 / 2}}-44090^{o}{ }_{3_{1 / 2}}\end{array}$ \\
\hline $\begin{array}{l}3730.358 \\
3730.836 \\
3731.412 \\
3731.735 \\
3735.501\end{array}$ & $\begin{array}{l}26799.45 \\
26796.02 \\
26791.88 \\
26789.56 \\
26762.56\end{array}$ & $\begin{array}{r}10 \\
60 \\
8 \\
500 \\
30\end{array}$ & $\begin{array}{l}1 \\
3\end{array}$ & $\begin{array}{l}20231_{1_{1 / 2}}-47030^{\circ}{ }_{1_{1 / 2}} \\
147333_{4_{1 / 2}}-41529^{\circ}{ }_{3_{1 / 2}} \\
16025_{5_{1 / 2}}-42817^{\circ}{ }_{{ }_{1 / 2}} \\
16287_{3_{1 / 2}}-43077^{\circ}{ }_{3_{1 / 2}} \\
17328_{4_{1 / 2}}-44090^{\circ}{ }_{3_{1 / 2}}\end{array}$ \\
\hline $\begin{array}{l}3735.753 \\
3737.420 \\
3738.501 \\
3740.988 \\
3742.789\end{array}$ & $\begin{array}{l}26760.75 \\
26748.82 \\
26741.08 \\
26723.30 \\
26710.45\end{array}$ & $\begin{array}{r}4 \\
300 \\
20 \\
2 \\
150\end{array}$ & 3 & $\begin{array}{l}198955_{2_{1 / 2}}-46656^{0}{ }_{3_{1 / 2}} \\
160255_{5_{1 / 2}}-42773^{0}{ }_{5_{1 / 2}} \\
115788_{1_{1 / 2}}-38319^{\circ}{ }_{{ }_{1 / 2}} \\
16287{ }_{3_{1 / 2}}-43011^{o}{ }_{2_{1 / 2}} \\
17146_{5_{1 / 2}}-43857^{0}{ }_{6_{1 / 2}}\end{array}$ \\
\hline $\begin{array}{l}3743.508 \\
3743.610 \\
3744.026 \\
3745.012 \\
3746.148\end{array}$ & $\begin{array}{l}26705.32 \\
26704.59 \\
26701.62 \\
26694.59 \\
26686.50\end{array}$ & $\begin{array}{r}1 \\
2 \\
150 \\
400 \\
1000\end{array}$ & $\begin{array}{r}2 \\
5 \\
10\end{array}$ & $\begin{array}{r}19903_{3_{1 / 2}}-46608^{o}{ }_{3_{1 / 2}} \\
20639_{5_{1 / 2}}-47343^{o}{ }_{4_{1 / 2}} \\
22180_{4_{1 / 2}}-48881^{o}{ }_{4_{1 / 2}} \\
15298_{3_{1 / 2}-41993^{o}{ }_{3_{1 / 2}}}-40689^{o}{ }_{0_{1 / 2}}\end{array}$ \\
\hline $\begin{array}{l}3746.640 \\
3746.845 \\
3749.634 \\
3749.928 \\
3750.566\end{array}$ & $\begin{array}{l}26682.99 \\
26681.53 \\
26661.69 \\
26659.60 \\
26655.06\end{array}$ & $\begin{array}{r}100 \\
5000 \\
10 \\
60 \\
6\end{array}$ & $\begin{array}{r}2 \\
150 \\
4\end{array}$ & $\begin{array}{r}161333_{4_{1 / 2}}-42817^{\circ}{ }_{4_{1 / 2}} \\
3700_{2_{1 / 2}}-30382^{o}{ }_{3_{1 / 2}} \\
16415_{2_{1 / 2}}-43077^{\circ}{ }^{3_{1 / 2}} \\
14169_{1_{1 / 2}}-40829^{\circ} 0_{0_{1 / 2}} \\
20639_{5_{1 / 2}}-47294^{o}{ }_{5_{1 / 2}}\end{array}$ \\
\hline $\begin{array}{l}3751.056 \\
3752.134 \\
3752.944 \\
3754.368 \\
3755.221\end{array}$ & $\begin{array}{l}26651.58 \\
26643.92 \\
26638.17 \\
26628.07 \\
26622.02\end{array}$ & $\begin{array}{r}6 \\
1000 \\
20 \\
4000 \\
80\end{array}$ & $\begin{array}{r}10 \\
40 \\
3\end{array}$ & $\begin{array}{r}14169_{1_{1 / 2}}-40821^{o}{ }_{2_{1 / 2}} \\
17328_{4_{1 / 2}}-43971^{\circ}{ }_{{ }_{1 / 2}} \\
115788_{1_{1 / 2}}-38216^{o}{ }_{{ }_{1 / 2}} \\
40022_{1_{1 / 2}}-30630^{\circ} o_{1_{1 / 2}} \\
15624_{2_{1 / 2}}-42246^{o}{ }_{3_{1 / 2}}\end{array}$ \\
\hline $\begin{array}{l}3757.722 \\
3758.535 \\
3758.948 \\
3758.948 \\
3759.305\end{array}$ & $\begin{array}{l}26604.30 \\
26598.55 \\
26595.63 \\
26595.63 \\
26593.10\end{array}$ & $\begin{array}{r}3 \\
1000 \\
5 \\
5 \\
5\end{array}$ & 10 & $\begin{array}{l}19791_{4_{1 / 2}}-46396^{o}{ }_{3_{1 / 2}} \\
15298_{3_{1 / 2}}-41897^{\circ}{ }_{4_{1 / 2}} \\
20686_{2_{1 / 2}}-47281^{o}{ }_{2_{1 / 2}} \\
16415_{2_{1 / 2}}-43011^{o} o_{2_{1 / 2}} \\
15624_{2_{1 / 2}}-42217^{\circ}{ }_{1_{1 / 2}}\end{array}$ \\
\hline $\begin{array}{l}3759.553 \\
3761.481 \\
3761.807 \\
3762.880 \\
3763.974\end{array}$ & $\begin{array}{l}26591.35 \\
26577.72 \\
26575.41 \\
26567.84 \\
26560.11\end{array}$ & $\begin{array}{r}20 \\
8 \\
2000 \\
6 \\
40\end{array}$ & $\begin{array}{c}1 \\
20 \\
1\end{array}$ & $\begin{array}{l}13252_{2_{1 / 2}}-39844^{o}{ }_{1_{1 / 2}} \\
17328_{4_{1 / 2}}-43905^{\circ}{ }_{3_{1 / 2}} \\
16025_{5_{1 / 2}}-42600^{\circ}{ }_{5_{1 / 2}} \\
21719_{5_{1 / 2}}-48287^{\circ} o_{5_{1 / 2}} \\
20783_{3_{1 / 2}}-47343^{\circ} o_{4_{1 / 2}}\end{array}$ \\
\hline $\begin{array}{l}3765.962 \\
3768.365 \\
3768.774 \\
3769.680 \\
3770.498\end{array}$ & $\begin{array}{l}26546.09 \\
26529.17 \\
26526.29 \\
26519.91 \\
26514.16\end{array}$ & $\begin{array}{r}2 \\
30 \\
5000 \\
3 \\
15\end{array}$ & 100 & $\begin{array}{r}20686_{2_{1 / 2}}-47232^{o}{ }_{2_{1 / 2}} \\
16287_{3_{1 / 2}}-42817^{\circ}{ }_{4_{1 / 2}} \\
4002_{1_{1 / 2}}-30528^{o}{ }_{2_{1 / 2}} \\
16025_{5_{1 / 2}}-42545^{o}{ }_{4_{1 / 2}} \\
22615_{3_{1 / 2}}-49130^{o}{ }_{3_{1 / 2}}\end{array}$ \\
\hline
\end{tabular}


TABLE 3. Classified lines of $\mathrm{TC} \mathrm{I}$

\begin{tabular}{|c|c|c|c|c|}
\hline \multirow{2}{*}{$\begin{array}{c}\text { Wavelength } \\
\text { A }\end{array}$} & \multirow{2}{*}{$\begin{array}{l}\text { Wavenumber } \\
\mathrm{cm}^{-1}\end{array}$} & \multicolumn{2}{|c|}{ Intensity } & \multirow{2}{*}{$\begin{array}{c}\text { Energy levels } \\
\mathrm{cm}^{-1}\end{array}$} \\
\hline & & Arc & Spark & \\
\hline $\begin{array}{l}3771.031 \\
3771.467 \\
3772.088 \\
3772.388 \\
3772.782\end{array}$ & $\begin{array}{l}26510.41 \\
26507.35 \\
26502.98 \\
26500.88 \\
26498.11\end{array}$ & $\begin{array}{r}3000 \\
4 \\
60 \\
40 \\
20\end{array}$ & $\begin{array}{l}50 \\
2 \\
2 \\
1\end{array}$ & $\begin{array}{r}41788_{0_{1 / 2}}-30689^{o} 0_{0_{1 / 2}} \\
20523{ }_{2_{1 / 2}}-47030^{o}{ }_{1_{1 / 2}} \\
171465_{5_{1 / 2}}-43649^{\circ}{ }_{4_{1 / 2}} \\
198955_{2_{1 / 2}}-46396^{o}{ }_{3_{1 / 2}} \\
20783_{3_{1 / 2}}-47281^{o}{ }_{{ }_{1 / 2}}\end{array}$ \\
\hline $\begin{array}{l}3773.413 \\
3773.930 \\
3774.783 \\
3774.970 \\
3775.355\end{array}$ & $\begin{array}{l}26493.68 \\
26490.05 \\
26484.06 \\
26482.75 \\
26480.05\end{array}$ & $\begin{array}{l}50 \\
1 \\
15 \\
10 \\
50 c\end{array}$ & 4 & $\begin{array}{l}21719_{5_{1 / 2}}-48213^{o}{ }_{4_{1 / 2}} \\
16415_{2_{1 / 2}}-42905^{o}{ }_{2_{1 / 2}} \\
16287_{3_{1 / 2}}-42771^{o}{ }_{3_{1 / 2}} \\
22615_{3_{1 / 2}}-49098^{o}{ }_{4_{1 / 2}} \\
16133_{4_{1 / 2}}-42613^{o}{ }_{4_{1 / 2}}\end{array}$ \\
\hline $\begin{array}{l}3775.560 \\
3777.274 \\
3779.373 \\
3779.693 \\
3782.299\end{array}$ & $\begin{array}{l}26478.61 \\
26466.60 \\
26451.90 \\
26449.66 \\
26431.43\end{array}$ & $\begin{array}{r}15 \\
500 \\
2000 \\
40 \\
15\end{array}$ & $\begin{array}{r}10 \\
40 \\
1\end{array}$ & $\begin{array}{r}15624_{2_{1 / 2}}-42102^{o} o_{2_{1 / 2}} \\
16133_{4_{1 / 2}}-42600^{o} \sigma_{5_{1 / 2}} \\
4178_{0_{1 / 2}}-30630^{o}{ }_{1_{1 / 2}} \\
16025_{5_{1 / 2}}-42474^{o}{ }_{6_{1 / 2}} \\
15298_{3_{1 / 2}}-41729^{o}{ }_{3_{1 / 2}}\end{array}$ \\
\hline $\begin{array}{l}3784.057 \\
3784.802 \\
3785.222 \\
3785.560 \\
3786.064\end{array}$ & $\begin{array}{l}26419.16 \\
26413.96 \\
26411.02 \\
26408.67 \\
26405.15\end{array}$ & $\begin{array}{r}500 \\
1 \\
20 \\
6 \\
200\end{array}$ & $\begin{array}{l}6 \\
1 \\
3\end{array}$ & $\begin{array}{l}16025_{5_{1 / 2}}-42444_{5_{1 / 2}}^{o} \\
21450_{4_{1 / 2}}-47864^{o} 3_{1 / 2} \\
16133_{4_{1 / 2}}-42545^{\circ} o_{1 / 2} \\
16287_{3_{1 / 2}}-42696^{\circ} o_{2_{1 / 2}} \\
15298_{3_{1 / 2}}-41703^{\circ} o_{4_{1 / 2}}\end{array}$ \\
\hline $\begin{array}{l}3786.494 \\
3787.772 \\
3788.154 \\
3788.370 \\
3791.282\end{array}$ & $\begin{array}{l}26402.15 \\
26393.24 \\
26390.58 \\
26389.08 \\
26368.81\end{array}$ & $\begin{array}{r}10 \\
1 \\
2 \\
1 \\
500\end{array}$ & 8 & $\begin{array}{l}19702_{1_{1 / 2}}-46104^{o} o_{1 / 2} \\
14679_{0_{1 / 2}}-41073^{o} o_{1 / 2} \\
21752_{3_{1 / 2}}-48142^{o} o_{1 / 2} \\
16133_{4_{1 / 2}}-42523^{\circ} 3_{1 / 2} \\
15624_{2_{1 / 2}}-41993^{\circ} o_{3_{1 / 2}}\end{array}$ \\
\hline $\begin{array}{l}3791.524 \\
3791.734 \\
3792.774 \\
3793.087 \\
3793.207\end{array}$ & $\begin{array}{l}26367.13 \\
26365.67 \\
26358.44 \\
26356.26 \\
26355.43\end{array}$ & $\begin{array}{r}3 \\
300 \\
1 \\
3 \\
1\end{array}$ & 7 & $\begin{array}{l}17282_{3_{1 / 2}}-43649^{\circ} o_{1 / 2} \\
17146_{5_{1 / 2}}-43512^{\circ} o_{5_{1 / 2}} \\
23455^{\circ}{ }_{2_{1 / 2}}-49813_{2_{1 / 2}} \\
16415_{2_{1 / 2}}-42771_{3_{1 / 2}}^{o} \\
14733_{4_{1 / 2}}-41088^{o} o_{3_{1 / 2}}\end{array}$ \\
\hline $\begin{array}{l}3794.800 \\
3796.522 \\
3797.435 \\
3797.767 \\
3798.055\end{array}$ & $\begin{array}{l}26344.36 \\
26332.42 \\
26326.09 \\
26323.78 \\
26321.79\end{array}$ & $\begin{array}{r}60 \\
12 \\
200 \\
1000 \\
40\end{array}$ & $\begin{array}{r}1 \\
5 \\
10 \\
1\end{array}$ & $\begin{array}{l}20686_{2_{1 / 2}}-47030^{o}{ }_{1_{1 / 2}} \\
15770_{1_{1 / 2}}-42102^{o}{ }_{2_{1 / 2}} \\
16287_{3_{1 / 2}}-42613^{o}{ }_{4_{1 / 2}} \\
1+252_{2_{1 / 2}}-39576^{o}{ }_{1_{1 / 2}} \\
17328_{4_{1 / 2}}-43649^{o}{ }_{4_{1 / 2}}\end{array}$ \\
\hline $\begin{array}{l}3799.692 \\
3803.976 \\
3804.534 \\
3806.144 \\
3806.424\end{array}$ & $\begin{array}{l}26310.45 \\
26280.82 \\
26276.96 \\
26265.85 \\
26263.92\end{array}$ & $\begin{array}{r}80 \\
40 \\
2 \\
15 \\
5\end{array}$ & $\begin{array}{l}2 \\
1\end{array}$ & $\begin{array}{l}161333_{4_{1 / 2}}-42444^{o} 5_{5_{1 / 2}} \\
164152_{2_{1 / 2}}-42696^{o} o_{2_{1 / 2}} \\
197911_{4_{1 / 2}}-46068^{o}{ }_{4_{1 / 2}} \\
226153_{3_{1 / 2}}-48881^{o}{ }_{4_{1 / 2}} \\
17282_{3_{1 / 2}}-43546^{0}{ }_{2_{1 / 2}}\end{array}$ \\
\hline $\begin{array}{l}3807.381 \\
3810.568 \\
3811.253 \\
3814.339 \\
3814.667\end{array}$ & $\begin{array}{l}26257.32 \\
26235.36 \\
26230.64 \\
26209.42 \\
26207.17\end{array}$ & $\begin{array}{r}10 \\
30 \\
80 \\
6 \\
200\end{array}$ & $\begin{array}{l}2 \\
2 h \\
3\end{array}$ & $\begin{array}{l}16287_{3_{1 / 2}}-42545^{o}{ }_{4_{1 / 2}} \\
16287_{3_{1 / 2}}-42523^{\circ} 3_{3_{1 / 2}} \\
15298_{3_{1 / 2}}-415290_{3_{1 / 2}} \\
19895_{2_{1 / 2}}-461040_{2_{1 / 2}}^{o} \\
13252_{2_{1 / 2}}-39459_{2_{1 / 2}}\end{array}$ \\
\hline $\begin{array}{l}3815.509 \\
3816.888 \\
3817.967 \\
3818.119 \\
3820.761\end{array}$ & $\begin{array}{l}26201.38 \\
26191.92 \\
26184.51 \\
26183.47 \\
26165.37\end{array}$ & $\begin{array}{r}80 \\
300 \\
150 \\
100 \\
50\end{array}$ & $\begin{array}{l}1 \\
4 \\
3 \\
2 \\
2\end{array}$ & 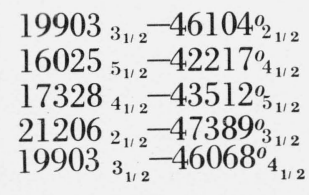 \\
\hline
\end{tabular}


TABLE 3. Classified lines of TC $\mathrm{I}$

\begin{tabular}{|c|c|c|c|c|}
\hline \multirow{2}{*}{$\begin{array}{c}\text { Wavelength } \\
\text { A }\end{array}$} & \multirow{2}{*}{$\begin{array}{l}\text { Wavenumber } \\
\mathrm{cm}^{-1}\end{array}$} & \multicolumn{2}{|c|}{ Intensity } & \multirow{2}{*}{$\begin{array}{l}\text { Energy levels } \\
\mathrm{cm}^{-1}\end{array}$} \\
\hline & & Arc & Spark & \\
\hline $\begin{array}{l}3822.672 \\
3823.066 \\
3824.467 \\
3825.531 \\
3826.013\end{array}$ & $\begin{array}{l}26152.29 \\
26149.59 \\
26140.01 \\
26132.74 \\
26129.45\end{array}$ & $\begin{array}{r}80 \\
1 \\
300 \\
5 \\
20\end{array}$ & 4 & $\begin{array}{l}15770_{1_{1 / 2}}-41922^{o}{ }_{2_{1 / 2}} \\
14679^{0_{1 / 2}}-40829^{\circ} o^{o} \\
15624^{2_{1 / 2}}-41764^{o}{ }_{{ }_{1 / 2}} \\
20523_{2_{1 / 2}}-46656^{o}{ }_{3_{1 / 2}} \\
14169_{1_{1 / 2}}-40299^{\circ}{ }_{2_{1 / 2}}\end{array}$ \\
\hline $\begin{array}{l}3827.593 \\
3828.537 \\
3828.750 \\
3829.220 \\
3830.352\end{array}$ & $\begin{array}{l}26118.66 \\
26112.22 \\
26110.77 \\
26107.57 \\
26099.85\end{array}$ & $\begin{array}{r}60 \\
500 \\
3 \\
150 \\
200\end{array}$ & $\begin{array}{l}1 \\
4\end{array}$ & $\begin{array}{l}17146_{5_{1 / 2}}-43265^{o}{ }_{4_{1 / 2}} \\
16133_{4_{1 / 2}}-42246^{o} o_{3_{1 / 2}} \\
23455^{0} o_{2_{1 / 2}}-49565_{2_{1 / 2}} \\
16415_{2_{1 / 2}}-42523^{0} o_{3_{1 / 2}} \\
21719_{5_{1 / 2}}-47819^{\circ}{ }_{4_{1 / 2}}\end{array}$ \\
\hline $\begin{array}{l}3831.883 \\
3832.448 \\
3832.817 \\
3837.559 \\
3839.577\end{array}$ & $\begin{array}{l}26089.42 \\
26085.58 \\
26083.07 \\
26050.84 \\
26037.15\end{array}$ & $\begin{array}{r}20 \\
200 \\
600 \\
1500 \\
1\end{array}$ & $\begin{array}{r}1 \\
5 \\
15\end{array}$ & $\begin{array}{l}21204_{4_{1 / 2}}-47294^{o}{ }_{5_{1 / 2}} \\
205233_{2_{1 / 2}}-46608^{\circ} o_{3_{1 / 2}} \\
161333_{4_{1 / 2}}-42217^{\circ}{ }^{\circ}{ }_{1 / 2} \\
171466_{5_{1 / 2}}-43197^{\circ} o_{6_{1 / 2}} \\
22180_{4_{1 / 2}}-48217^{\circ} o_{3_{1 / 2}}\end{array}$ \\
\hline $\begin{array}{l}3840.137 \\
3841.307 \\
3842.342 \\
3843.968 \\
3844.061\end{array}$ & $\begin{array}{l}26033.35 \\
26025.42 \\
26018.41 \\
26007.40 \\
26006.77\end{array}$ & $\begin{array}{r}60 \\
800 \\
80 \\
100 \\
20\end{array}$ & $\begin{array}{l}8 \\
2\end{array}$ & $\begin{array}{l}22180_{4_{1 / 2}}-48213^{o}{ }_{4_{1 / 2}} \\
17146_{5_{1 / 2}}-43172^{o} 5_{5_{1 / 2}} \\
15770_{1_{1 / 2}}-41788^{o}{ }_{1_{1 / 2}} \\
16025_{5_{1 / 2}}-42032^{o} o_{5_{1 / 2}} \\
16287_{3_{1 / 2}}-42294^{o} o_{2_{1 / 2}}\end{array}$ \\
\hline $\begin{array}{l}3844.347 \\
3845.974 \\
3847.351 \\
3847.596 \\
3849.519\end{array}$ & $\begin{array}{l}26004.84 \\
25993.84 \\
25984.54 \\
25982.88 \\
25969.90\end{array}$ & $\begin{array}{r}60 \\
800 \\
100 \\
500 \\
1\end{array}$ & $\begin{array}{l}1 \\
8 \\
1 \\
3\end{array}$ & $\begin{array}{l}21447_{1_{1 / 2}}-47452^{o}{ }_{2_{1 / 2}} \\
15770_{1_{1 / 2}}-41764^{o} 2_{2_{1 / 2}} \\
17282_{3_{1 / 2}}-43267^{\circ} o_{1 / 2} \\
17282_{3_{1 / 2}}-43265^{o} 4_{1 / 2} \\
20686_{2_{1 / 2}}-46656^{o} o_{3_{1 / 2}}\end{array}$ \\
\hline $\begin{array}{l}3850.553 \\
3851.222 \\
3852.328 \\
3853.323 \\
3854.074\end{array}$ & $\begin{array}{l}25962.93 \\
25958.42 \\
25950.97 \\
25944.26 \\
25939.21\end{array}$ & $\begin{array}{r}5 \\
300 \\
80 \\
2 \\
8\end{array}$ & $\begin{array}{l}3 \\
1\end{array}$ & $\begin{array}{l}16287_{3_{1 / 2}}-42250^{\circ} o_{2_{1 / 2}} \\
16287_{3_{1 / 2}}-4246^{\circ} o_{3_{1 / 2}} \\
17146_{5_{1 / 2}}-43097^{\circ} 0_{1 / 2} \\
21920_{2_{1 / 2}}-47864^{\circ} 3_{3_{1 / 2}} \\
17328_{4_{1 / 2}}-43260^{o_{1 / 2}}\end{array}$ \\
\hline $\begin{array}{l}3854.326 \\
3855.555 \\
3855.914 \\
3856.543 \\
3856.734\end{array}$ & $\begin{array}{l}25937.51 \\
25929.25 \\
25926.83 \\
25922.60 \\
25921.32\end{array}$ & $\begin{array}{r}50 \\
20 \\
1 \\
20 \\
500 c\end{array}$ & $5 h$ & $\begin{array}{l}17328_{4_{1 / 2}}-43265^{0}{ }_{4_{1 / 2}} \\
16287_{3_{1 / 2}}-42217^{\circ}{ }_{4_{1 / 2}} \\
14169_{1_{1 / 2}}-40096^{o}{ }_{1_{1 / 2}} \\
20686_{{ }_{1 / 2}}-46608^{o} o_{3_{1 / 2}} \\
16553{ }_{6_{1 / 2}}-42474^{o}{ }_{6_{1 / 2}}\end{array}$ \\
\hline $\begin{array}{l}3857.388 \\
3860.129 \\
3861.259 \\
3863.068 \\
3863.830\end{array}$ & $\begin{array}{l}25916.93 \\
25898.52 \\
25890.94 \\
25878.82 \\
25873.72\end{array}$ & $\begin{array}{r}20 \\
8 \\
25 c \\
200 \\
100\end{array}$ & $\begin{array}{l}3 \\
1\end{array}$ & $\begin{array}{l}19791_{4_{1 / 2}}-45708^{o}{ }_{4_{1 / 2}} \\
161333_{4_{1 / 2}}-42032^{o} 5_{1 / 2} \\
16553{ }_{6_{1 / 2}}-424444^{o} o_{1 / 2} \\
16415{ }_{2_{1 / 2}}-42294^{o} o_{2_{1 / 2}} \\
20231_{1_{1 / 2}}-461040_{2_{1 / 2}}^{o}\end{array}$ \\
\hline $\begin{array}{l}3864.106 \\
3866.012 \\
3866.608 \\
3868.240 \\
3870.292\end{array}$ & $\begin{array}{l}25871.87 \\
25859.11 \\
25855.13 \\
25844.22 \\
25830.52\end{array}$ & $\begin{array}{r}400 \\
30 \\
5 \\
1000 \\
10\end{array}$ & 4 & $\begin{array}{l}160255_{5_{1 / 2}}-41897^{\circ} o_{4_{1 / 2}} \\
161333_{4_{1 / 2}}-41993^{\circ} 3_{3_{1 / 2}} \\
15770_{1_{1 / 2}}-41625^{\circ}{ }_{1_{1 / 2}} \\
17328_{4_{1 / 2}}-43172^{o} o_{5_{1 / 2}} \\
16415_{2_{1 / 2}}-42246^{o} o_{3_{1 / 2}}\end{array}$ \\
\hline $\begin{array}{l}3871.055 \\
3871.223 \\
3874.609 \\
3875.658 \\
3876.366\end{array}$ & $\begin{array}{l}25825.43 \\
25824.31 \\
25801.74 \\
25794.75 \\
25790.04\end{array}$ & $\begin{array}{r}12 \\
12 \\
12 \\
200 \\
80\end{array}$ & $\begin{array}{l}2 \\
1\end{array}$ & $\begin{array}{l}25598_{2_{1 / 2}}-51423^{o}{ }_{3_{1 / 2}} \\
21206_{2_{1 / 2}}-47030^{\circ}{ }_{1_{1 / 2}} \\
16415_{2_{1 / 2}}-42217^{\circ}{ }_{1_{1 / 2}} \\
17282_{3_{1 / 2}}-43077^{0} 3_{3_{1 / 2}} \\
15298_{3_{1 / 2}}-41088^{o}{ }_{3_{1 / 2}}\end{array}$ \\
\hline
\end{tabular}


TABLE 3. Classified lines of Tc I

\begin{tabular}{|c|c|c|c|c|}
\hline \multirow{2}{*}{$\begin{array}{c}\text { Wavelength } \\
\text { A }\end{array}$} & \multirow{2}{*}{$\begin{array}{c}\text { Wavenumber } \\
\mathrm{cm}^{-1}\end{array}$} & \multicolumn{2}{|c|}{ Intensity } & \multirow{2}{*}{$\begin{array}{c}\text { Energy levels } \\
\mathrm{cm}^{-1}\end{array}$} \\
\hline & & Arc & Spark & \\
\hline 3877.139 & 25784.90 & 30 & & $21447_{1_{1 / 2}}-47232_{2_{1 / 2}}^{o}$ \\
\hline 3882.497 & 25749.32 & 10 & & $17328_{4_{1 / 2}}-43077^{0} 0_{3_{1 / 2}}$ \\
\hline 3885.638 & 25728.50 & 3 & & $17282_{3_{1 / 2}}-43011_{2_{1 / 2}}^{o_{1 / 2}}$ \\
\hline 3886.896 & 25720.18 & 2 & & $20384_{3_{1 / 2}}-46104_{2_{1 / 2}}$ \\
\hline 3888.177 & 25711.70 & $1 h$ & & $23265^{\circ}{ }_{3_{1 / 2}}-48977_{4_{1 / 2}}$ \\
\hline 3888.440 & 25709.96 & 80 & 1 & $20686_{2_{1 / 2}}-46396_{3_{1 / 2}}^{o}$ \\
\hline 3889.130 & 25705.40 & 150 & 2 & $16287_{3_{1 / 2}^{1 / 2}}-41993_{3_{1 / 2}}^{o_{1 / 2}}$ \\
\hline 3892.332 & 25684.26 & 100 & 1 & $20384_{3_{1 / 2}}-46068^{\circ}{ }_{4_{1 / 2}}$ \\
\hline 3892.332 & 25684.26 & 100 & 1 & $22180_{4_{1 / 2}}^{0,4 / 2}-4864_{3_{1 / 2}}^{o_{1 / 2}}$ \\
\hline 3893.220 & 25678.40 & 200 & 2 & $16025_{5_{1 / 2}}-41703^{o}{ }_{4_{1 / 2}}$ \\
\hline 3893.837 & 25674.33 & 4 & & $14169_{1_{1 / 2}}-39844^{o} o_{1_{1 / 2}}$ \\
\hline 3894.482 & 25670.08 & 15 & & $14733_{4_{1 / 2}}-40403^{\circ}{ }_{1 / 2}$ \\
\hline 3894.482 & 25670.08 & 15 & & $17146_{5_{1 / 2}}-42817^{\circ} 4_{1 / 2}$ \\
\hline 3894.688 & 25668.72 & 2 & & $24147_{4_{1 / 2}}^{0}-49815^{0}{ }_{4_{1 / 2}}^{t_{1 / 2}}$ \\
\hline 3899.120 & 25639.54 & 100 & 1 & $22180_{4_{1 / 2}}-47819^{\circ}{ }_{4_{1 / 2}}$ \\
\hline 3899.827 & 25634.90 & 600 & 5 & $16287_{3_{1 / 2}}-41922_{2_{1 / 2}}$ \\
\hline 3901.473 & 25624.08 & 5 & & $21719_{5_{1 / 2}}-47343^{\circ} 0_{1 / 2}^{1 / 2}$ \\
\hline 3903.740 & 25609.20 & 60 & & $16287_{3_{1 / 2}}-41897^{\circ}{ }_{1 / 2}$ \\
\hline 3905.322 & 25598.83 & 8 & & $22265_{2_{1 / 2}}-47864^{o_{1 / 2}}$ \\
\hline 3905.517 & 25597.55 & 30 & & $22615_{3_{1 / 2}}-48213_{4_{1 / 2}}^{o}$ \\
\hline 3905.774 & 25595.86 & 100 & 1 & $16133_{4_{1 / 2}}-41729^{o}{ }_{3_{1 / 2}}$ \\
\hline 3906.400 & 25591.76 & 20 & & $21752_{3_{1 / 2}}-47343^{o}{ }_{1 / 2}$ \\
\hline 3907.681 & 25583.37 & 1 & & $21447_{1_{1 / 2}}-47030^{\circ}{ }_{1_{1 / 2}}$ \\
\hline 3907.958 & 25581.56 & 30 & & $20523_{2_{1 / 2}}^{1 / 2}-46104_{2_{1 / 2}}^{o_{1 / 2}}$ \\
\hline 3908.596 & 25577.39 & 20 & & $16415_{2_{1 / 2}}-41993_{3_{1 / 2}}^{o}$ \\
\hline 3909.038 & 25574.49 & 8 & & $21719_{5_{1 / 2}}-47294_{5_{1 / 2}}^{o}$ \\
\hline 3909.782 & 25569.63 & 20 & & $16133_{4_{1 / 2}}-41703^{\circ} 4_{1 / 2}$ \\
\hline 3911.921 & 25555.65 & 3 & & $16025_{5_{1 / 2}}-41580^{\circ} 5_{1 / 2}$ \\
\hline 3915.174 & 25534.41 & 2 & & $17282_{3_{1 / 2}}-42817^{\circ}{ }_{4_{1 / 2}}$ \\
\hline 3915.889 & 25529.75 & 10 & & $21752_{3_{1 / 2}}-47281_{2_{1 / 2}}$ \\
\hline 3916.536 & 25525.53 & 60 & 1 & $21926_{2_{1 / 2}}-47452_{2_{1 / 2}}^{o}$ \\
\hline 3916.941 & 25522.89 & 5 & & $15298_{3_{1 / 2}}^{1 / 2}-40821^{\circ} o_{1 / 2}^{1 / 2}$ \\
\hline 3917.443 & 25519.62 & $10 c$ & & $23265^{\circ}{ }_{3_{1 / 2}}-48784_{3_{1 / 2}}$ \\
\hline 3919.148 & 25508.52 & 50 & & $14733_{4_{1 / 2}}^{0}-40241_{5_{1 / 2}}^{0}$ \\
\hline 3919.375 & 25507.04 & 300 & 3 & $16415_{2_{1 / 2}}-41922_{2_{1 / 2}}^{o}$ \\
\hline 3922.134 & 25489.10 & 100 & 1 & $17282_{3_{1 / 2}}-42771_{3_{1 / 2}}^{o}$ \\
\hline 3922.134 & 25489.10 & 100 & 1 & $17328_{4_{1 / 2}}-42817^{\circ}{ }_{4_{1 / 2}}$ \\
\hline 3923.663 & 25479.17 & $300 c$ & $3 c$ & $16553_{6_{1 / 2}}-42032^{o} 5_{1 / 2}$ \\
\hline 3925.143 & 25469.56 & 10 & & $21920_{2_{1 / 2}}-47389_{3_{1 / 2}}$ \\
\hline 3925.953 & 25464.31 & 20 & & $15624_{2_{1 / 2}}-41088_{3_{1 / 2}}$ \\
\hline 3927.573 & 25453.80 & 200 & 2 & $17146_{5_{1 / 2}}-42600^{\circ} 5_{1 / 2}$ \\
\hline 3928.323 & 25448.95 & 10 & & $15624_{2_{1 / 2}}-41073^{o}{ }_{1 / 2}^{1 / 2}$ \\
\hline 3928.770 & 25446.05 & 30 & & $17328_{4_{1 / 2}}-42773^{o} 5_{1 / 2}$ \\
\hline 3929.113 & 25443.83 & 10 & & $17328_{4_{1 / 2}}-42771_{3_{1 / 2}}$ \\
\hline 3929.378 & 25442.11 & 40 & & $16287_{3_{1 / 2}}-41729^{o_{3_{1 / 2}}}$ \\
\hline 3931.319 & 25429.55 & 3 & & $20639_{5_{1 / 2}}-46068^{o}{ }_{4_{1 / 2}}$ \\
\hline 3933.438 & 25415.85 & 150 & 2 & $16287_{3_{1 / 2}}-41703^{\circ}{ }_{4_{1 / 2}}$ \\
\hline 3934.832 & 25406.85 & 30 & & $14169_{1_{1 / 2}}-39576^{o} o_{1 / 2}$ \\
\hline 3936.175 & 25398.18 & 1 & & $17146_{5_{1 / 2}}-42545^{\circ}{ }_{4_{1 / 2}}$ \\
\hline 3936.648 & 25395.13 & 70 & & $16133_{4 / 2}^{1 / 2}-41529^{\circ} 0_{1 / 2}^{1 / 2}$ \\
\hline
\end{tabular}


TABLE 3. Classified lines of $\mathrm{Tc} \mathrm{I}$

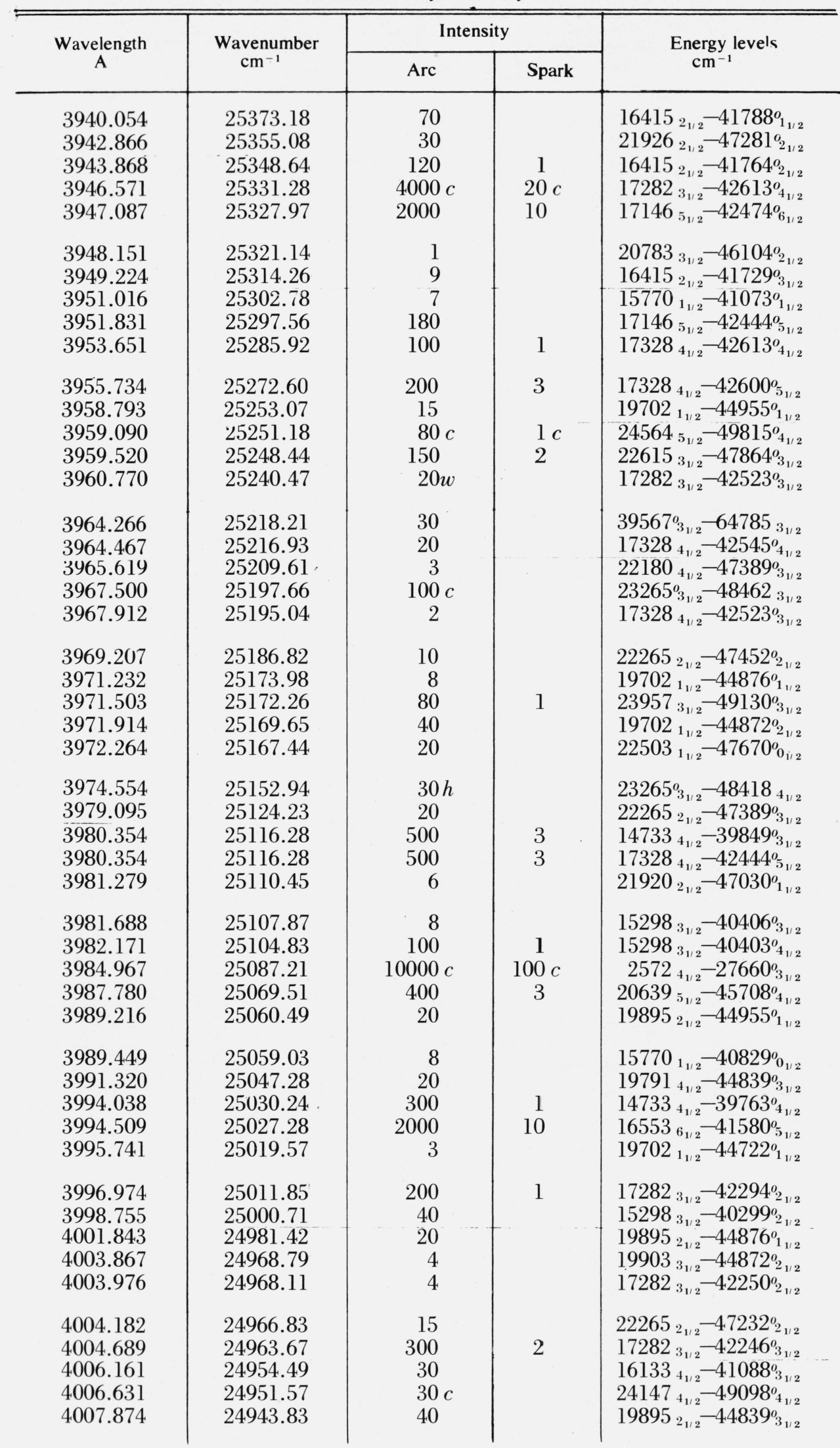


TABLE 3. Classified lines of TC I

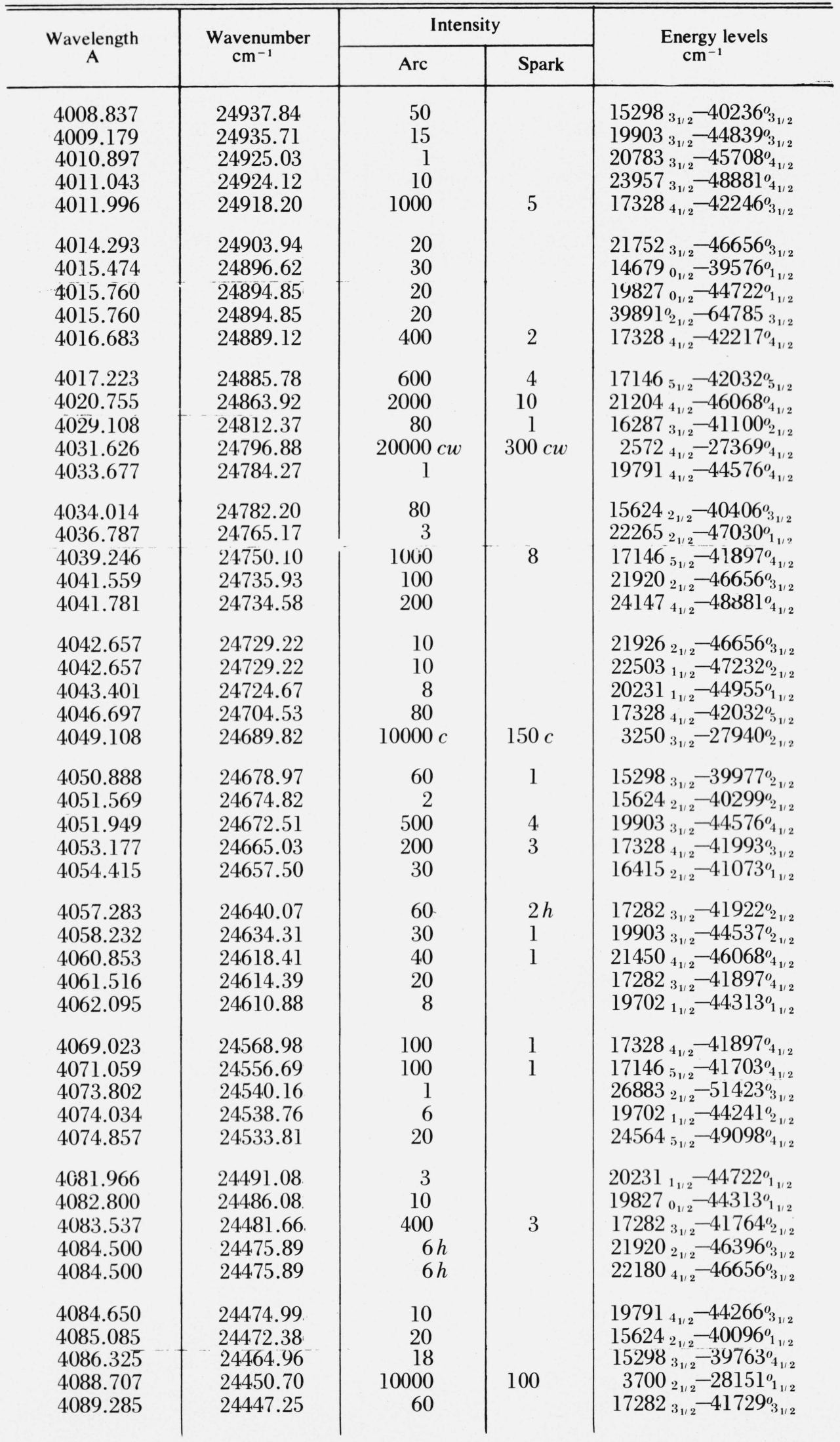


TABLE 3. Classified lines of Tc I

\begin{tabular}{|c|c|c|c|c|}
\hline \multirow{2}{*}{$\begin{array}{c}\text { Wavelength } \\
\text { A }\end{array}$} & \multirow{2}{*}{$\begin{array}{l}\text { Wavenumber } \\
\mathrm{cm}^{-1}\end{array}$} & \multicolumn{2}{|c|}{ Intensity } & \multirow{2}{*}{$\begin{array}{l}\text { Energy levels } \\
\mathrm{cm}^{-1}\end{array}$} \\
\hline & & Arc & Spark & \\
\hline 4092.373 & 24428.80 & 12 & & $22180_{4_{1 / 2}}-46608_{3_{1 / 2}}$ \\
\hline 4093.694 & 24420.92 & 200 & 2 & $17282_{3_{1 / 2}}-41703_{4_{1 / 2}}^{o_{1 / 2}}$ \\
\hline 4094.163 & 24418.12 & 80 & 1 & $19895_{2_{1 / 2}}-44313_{1_{1 / 2}}^{o}$ \\
\hline 4095.668 & 24409.15 & 15000 & 500 & $3250_{3_{1 / 2}}-27660_{3_{1 / 2}}^{o_{1 / 2}}$ \\
\hline 4098.784 & 24390.59 & 100 & 1 & $22265_{2_{1 / 2}}-46656^{o} o_{1 / 2}^{1 / 2}$ \\
\hline 4100.114 & 24382.68 & $150 \mathrm{cw}$ & \multirow{5}{*}{1} & $40403_{4_{1 / 2}}-64785_{3_{1 / 2}}$ \\
\hline 4100.902 & 24377.99 & 20 & & $16025_{5_{1 / 2}}-40403_{4_{1 / 2}}$ \\
\hline 4101.312 & 24375.56 & 100 & & $17328_{4_{1 / 2}}-41703^{\circ} o_{1 / 2}^{1 / 2}$ \\
\hline 4101.989 & 24371.54 & 30 & & $19895_{2_{1 / 2}}-44266_{3_{1 / 2}}^{o_{1 / 2}}$ \\
\hline 4103.368 & 24363.34 & 10 & & $19903_{3_{1 / 2}}-44266_{3_{1 / 2}^{0}}^{0}$ \\
\hline 4103.987 & 24359.67 & 3 & \multirow[b]{5}{*}{1} & $23460_{3_{1 / 2}}-47819_{4_{1 / 2}}^{o}$ \\
\hline 4105.089 & 24353.13 & 80 & & $15624_{2_{1 / 2}}-39977_{2_{1 / 2}}^{1 / 2}$ \\
\hline 4106.246 & 24346.27 & 60 & & $19791_{4_{1 / 2}}-44138_{5_{1 / 2}} o_{1 / 2}$ \\
\hline 4106.246 & 24346.27 & 60 & & $19895_{2_{1 / 2}}-442410_{2_{1 / 2}}^{o_{1 / 2}}$ \\
\hline 4106.723 & 24343.44 & 80 & & $22265_{2_{1 / 2}}^{-1 / 2}-46608_{3_{1 / 2}}^{1 / 2}$ \\
\hline 4107.644 & 24337.98 & 20 & \multirow{5}{*}{1} & $19903_{3_{1 / 2}}-44241_{2_{1 / 2}}$ \\
\hline 4109.646 & 24326.13 & 40 & & $15770_{1_{1 / 2}}^{1 / 2}-40096_{1_{1 / 2}}^{o}$ \\
\hline 4111.199 & 24316.94 & 6 & & $24564_{5_{1 / 2}}-48881_{4_{1 / 2}}^{0}$ \\
\hline 4111.386 & 24315.83 & 150 & & $20523_{2_{1 / 2}}-44839^{\circ}{ }_{3_{1 / 2}}^{1 / 2}$ \\
\hline 4113.341 & 24304.28 & 30 & & $19702_{1_{1 / 2}}-44007_{2_{1 / 2}}^{\circ}$ \\
\hline 4115.077 & 24294.02 & 10000 & \multirow[t]{5}{*}{300} & $4002_{1_{1 / 2}}-28296_{0}^{o}$ \\
\hline 4117.021 & 24282.55 & 4 & & $19827_{0_{1 / 2}}-44110^{o}{ }_{1_{1 / 2}}$ \\
\hline 4118.740 & 24272.42 & 6 & & $16133_{4_{1 / 2}}-40406^{o} o_{3_{1 / 2}}$ \\
\hline 4119.274 & 24269.27 & 600 & & $15298_{3_{1 / 2}}-39567^{\circ} 0_{1 / 2}$ \\
\hline 4121.576 & 24255.72 & 100 & & $23957_{3_{1 / 2}}-48213_{4_{1 / 2}}$ \\
\hline 4124.217 & 24240.18 & 8000 & \multirow{2}{*}{$\begin{array}{r}200 \\
20\end{array}$} & $3700_{2_{1 / 2}}-27940^{\circ}{ }_{2_{1 / 2}}$ \\
\hline 1128.268 & 24216.40 & 1000 & & $16025_{5_{1 / 2}}^{1 / 2}-40241_{5_{1 / 2}}^{o / / 2}$ \\
\hline 4128.557 & 24214.70 & 80 & \multirow[t]{2}{*}{1} & $19895_{2_{1 / 2}}-44110^{o} 1_{1 / 2}^{1 / 2}$ \\
\hline 4129.862 & 24207.05 & 6 & & $15770_{1_{1 / 2}}-39977^{\circ} o_{1 / 2}$ \\
\hline 4130.453 & 24203.59 & 150 & \multirow[t]{6}{*}{2} & $19903_{3_{1 / 2}}-441070_{2_{1 / 2}}$ \\
\hline 4130.877 & 24201.10 & 30 & & $17328_{4_{1 / 2}}-41529^{o}{ }_{3_{1 / 2}}$ \\
\hline 4132.530 & 24191.42 & 40 & & $20384_{3_{1 / 2}}-44576^{0}{ }_{4_{1 / 2}}$ \\
\hline 4133.216 & 24187.06 & 20 & & $19903_{3_{1 / 2}}-44090^{\circ} 0_{1 / 2}$ \\
\hline 4133.679 & 24184.70 & 2 & & $21920_{2_{1 / 2}}-46104_{2_{1 / 2}}^{o}$ \\
\hline 4134.490 & 24179.96 & 3 & & $19791_{4_{1 / 2}}-43971_{4_{1 / 2}}^{o}$ \\
\hline 4134.813 & 24178.07 & 300 & \multirow[t]{3}{*}{3} & $21926_{2_{1 / 2}}-46104_{2_{1 / 2}}^{o}$ \\
\hline 4137.234 & 24163.92 & 15 & & $20523_{2_{1 / 2}}-44687^{\circ} 3_{1 / 2}^{1 / 2}$ \\
\hline 4137.660 & 24161.43 & 4 & & $15298_{3_{1 / 2}}-39459^{\circ} 0_{1 / 2}$ \\
\hline 4139.116 & 24152.93 & 300 & \multirow{2}{*}{$\begin{array}{l}3 \\
8\end{array}$} & $20384_{3_{1 / 2}}-44537_{2_{1 / 2}}^{-1 / 2}$ \\
\hline 4139.849 & 24148.66 & 800 & & $4002_{1_{1 / 2}}-28151_{1_{1 / 2}}^{o}$ \\
\hline 4142.917 & 24130.77 & 20 & \multirow{5}{*}{$\begin{array}{r}30 \\
15 \\
1\end{array}$} & $22265_{2_{1 / 2}}-46396_{3_{1 / 2}}^{0}$ \\
\hline 4144.950 & 24118.94 & 6000 & & $3250_{3_{1 / 2}}^{1 / 2}-27369^{\circ}{ }_{4_{1 / 2}}$ \\
\hline 4145.080 & 24118.18 & 3000 & & $4178_{0_{1 / 2}}^{0}-28296^{\circ} 0_{1 / 2}^{1 / 2}$ \\
\hline 4146.202 & 24111.65 & 80 & & $19895_{2_{1 / 2}}^{1 / 2}-44007^{0} 0_{2_{1 / 2}}{ }^{1 / 2}$ \\
\hline 4146.882 & 24107.70 & 20 & & $16133_{4_{1 / 2}}-40241_{5_{1 / 2}}$ \\
\hline 4147.615 & 24103.44 & 200 & \multirow[t]{3}{*}{2} & $19903_{3_{1 / 2}}-44007_{2_{1 / 2}}$ \\
\hline 4150.200 & 24088.43 & 15 & & $20783_{3_{1 / 2}}-44872_{2_{1 / 2}}^{o}$ \\
\hline 4151.241 & 24082.39 & 10 & & $20231_{1_{1 / 2}}-44313^{o} 1_{1 / 2}$ \\
\hline 4153.672 & 24068.29 & 150 & \multirow[t]{2}{*}{1} & $19903_{3_{1 / 2}}-43971_{4_{1 / 2}}^{1 / 2}$ \\
\hline 4158.547 & 24040.08 & 10 & & $22615_{3_{1 / 2}}-46656_{3_{1 / 2}}^{0}$ \\
\hline
\end{tabular}


TABLE 3. Classified lines of $\mathrm{Tc} \mathrm{I}$

\begin{tabular}{|c|c|c|c|c|}
\hline \multirow{2}{*}{$\begin{array}{c}\text { Wavelength } \\
\text { A }\end{array}$} & \multirow{2}{*}{$\begin{array}{l}\text { Wavenumber } \\
\mathrm{cm}^{-1}\end{array}$} & \multicolumn{2}{|c|}{ Intensity } & \multirow{2}{*}{$\begin{array}{l}\text { Energy levels } \\
\mathrm{cm}^{-1}\end{array}$} \\
\hline & & Arc & Spark & \\
\hline $\begin{array}{l}4158.547 \\
4159.319 \\
4162.970 \\
4163.702 \\
4165.609\end{array}$ & $\begin{array}{l}24040.08 \\
24035.62 \\
24014.54 \\
24010.32 \\
23999.32\end{array}$ & $\begin{array}{r}10 \\
20 \\
1 \\
20 \\
10000\end{array}$ & 100 & $\begin{array}{l}25058_{3_{1 / 2}}-49098^{o}{ }_{4_{1 / 2}} \\
39891_{2_{1 / 2}}-63926_{2_{1 / 2}}^{o^{2}} \\
20523_{2_{1 / 2}}-44537^{\circ} o_{2_{1 / 2}} \\
19895_{2_{1 / 2}}-43905^{o}{ }_{3_{1 / 2}} \\
10516_{3_{1 / 2}}-34515_{2_{1 / 2}}^{o}\end{array}$ \\
\hline $\begin{array}{l}4167.424 \\
4170.274 \\
4172.532 \\
4174.462 \\
4176.276\end{array}$ & $\begin{array}{l}23988.87 \\
23972.48 \\
23959.50 \\
23948.43 \\
23938.03\end{array}$ & $\begin{array}{r}500 \\
4000 \\
5000 \\
70 \\
1000\end{array}$ & $\begin{array}{r}6 \\
40 \\
60 \\
50\end{array}$ & $\begin{array}{r}21719_{5_{1 / 2}}-45708^{o}{ }_{4_{1 / 2}} \\
4178_{0_{1 / 2}}-28151^{o} o_{1_{1 / 2}} \\
3700_{2_{1 / 2}}-27660^{\circ}{ }_{3_{1 / 2}} \\
16287_{3_{1 / 2}}-40236^{0} o_{3_{1 / 2}} \\
4002_{1_{1 / 2}}-27940^{0} o_{2_{1 / 2}}\end{array}$ \\
\hline $\begin{array}{l}4182.629 \\
4185.815 \\
4186.506 \\
4189.609 \\
4190.306\end{array}$ & $\begin{array}{l}23901.67 \\
23883.48 \\
23879.53 \\
23861.85 \\
23857.88\end{array}$ & $\begin{array}{r}5 \\
3 \\
800 \\
50 \\
2\end{array}$ & 30 & $\begin{array}{l}19903_{3_{1 / 2}}-43805^{o} o_{3_{1 / 2}} \\
16415_{2_{1 / 2}}-40299^{\circ} o_{2_{1 / 2}} \\
11063_{2_{1 / 2}}-34942^{\circ}{ }_{1_{1 / 2}} \\
23957{ }_{3_{1 / 2}}-47819^{\circ}{ }_{4_{1 / 2}} \\
19791_{4_{1 / 2}}-43649^{\circ}{ }_{4_{1 / 2}}\end{array}$ \\
\hline $\begin{array}{l}4190.477 \\
4191.415 \\
4193.557 \\
4194.224 \\
4199.466\end{array}$ & $\begin{array}{l}23856.90 \\
23851.57 \\
23839.38 \\
23835.59 \\
23805.84\end{array}$ & $\begin{array}{r}4 \\
10 \\
1 \\
3 \\
30\end{array}$ & 1 & $\begin{array}{l}203844_{3_{1 / 2}}-442410_{2_{1 / 2}}^{o} \\
206866_{2_{1 / 2}}-445370_{2_{1 / 2}}^{o} \\
222655_{2_{1 / 2}}-461040_{2_{1 / 2}} \\
156242_{2_{1 / 2}}-39459^{\circ} o_{2_{1 / 2}} \\
17282_{3_{1 / 2}}-41088^{\circ} o_{3_{1 / 2}}\end{array}$ \\
\hline $\begin{array}{l}4201.850 \\
4211.430 \\
4214.220 \\
4215.510 \\
4218.609\end{array}$ & $\begin{array}{l}23792.33 \\
23738.21 \\
23722.50 \\
23715.24 \\
23697.82\end{array}$ & $\begin{array}{r}20 \\
20 \\
5 \\
10 \\
300\end{array}$ & $\begin{array}{r}1 \\
1 \\
1 \\
1 \\
20\end{array}$ & $\begin{array}{l}207833_{3_{1 / 2}}-44576^{o}{ }_{4_{1 / 2}} \\
160255_{5_{1 / 2}}-39763^{o}{ }_{4_{1 / 2}} \\
20384_{3_{1 / 2}}-44107^{0} o_{2_{1 / 2}} \\
161333_{4_{1 / 2}}-39849^{0} o_{3_{1 / 2}} \\
11578{ }_{1_{1 / 2}}-35276^{0} 0_{0_{1 / 2}}\end{array}$ \\
\hline $\begin{array}{l}4220.346 \\
4221.619 \\
4229.948 \\
4230.207 \\
4230.832\end{array}$ & $\begin{array}{l}23688.06 \\
23680.92 \\
23634.29 \\
23632.85 \\
23629.35\end{array}$ & $\begin{array}{c}50 h \\
10 \\
6 \\
10 \\
150\end{array}$ & $\begin{array}{l}1 \\
5\end{array}$ & 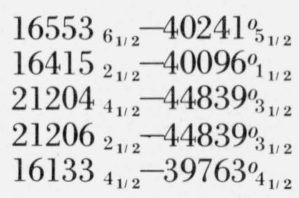 \\
\hline $\begin{array}{l}4231.195 \\
4232.096 \\
4238.191 \\
4238.991 \\
4239.567\end{array}$ & $\begin{array}{l}23627.33 \\
23622.30 \\
23588.33 \\
23583.87 \\
23580.67\end{array}$ & $\begin{array}{c}5 \\
40 \\
5000 c \\
5 \\
20\end{array}$ & $\begin{array}{c}1 \\
100 c \\
1\end{array}$ & 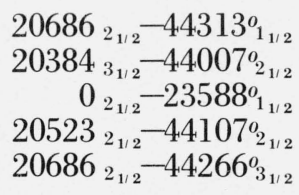 \\
\hline $\begin{array}{l}4241.996 \\
4242.979 \\
4248.953 \\
4252.756 \\
4256.134\end{array}$ & $\begin{array}{l}23567.17 \\
23561.71 \\
23528.58 \\
23507.54 \\
23488.88\end{array}$ & $\begin{array}{c}8 \\
40 \\
30 \\
2 h \\
10\end{array}$ & $\begin{array}{l}1 \\
1\end{array}$ & $\begin{array}{l}205232_{2_{1 / 2}}-44090^{o}{ }_{3_{1 / 2}} \\
16415{ }_{2_{1 / 2}}-39977^{\circ} o_{1 / 2} \\
22180_{4_{1 / 2}}-45708^{o} o_{1 / 2} \\
147333_{4_{1 / 2}}-38240^{o} o_{3_{1 / 2}} \\
226153_{3_{1 / 2}}-46104_{2_{1 / 2}}^{o}\end{array}$ \\
\hline $\begin{array}{l}4257.338 \\
4258.587 \\
4258.587 \\
4262.270 \\
4262.687\end{array}$ & $\begin{array}{l}23482.24 \\
23475.35 \\
23475.35 \\
23455.07 \\
23452.78\end{array}$ & $\begin{array}{r}30 \\
20 \\
20 \\
10000 \\
1000\end{array}$ & $\begin{array}{r}1 \\
1 \\
1 \\
400 \\
20\end{array}$ & $\begin{array}{r}21204_{4_{1 / 2}}-44687^{\circ} o_{3_{1 / 2}} \\
16415_{2_{1 / 2}}-39891_{2_{1 / 2}}^{\circ} \\
19791_{2_{1 / 2}}-43267^{0_{1 / 2}} \\
0_{2_{1 / 2}}-23455^{\circ} o_{2_{1 / 2}} \\
11063_{2_{1 / 2}}-34515_{2_{1 / 2}}^{\circ}\end{array}$ \\
\hline $\begin{array}{l}4266.158 \\
4266.158 \\
4266.983 \\
4267.978 \\
4268.503\end{array}$ & $\begin{array}{l}23433.69 \\
23433.69 \\
23429.16 \\
23423.70 \\
23420.82\end{array}$ & $\begin{array}{r}80 \\
80 \\
2 \\
8 \\
30\end{array}$ & $\begin{array}{l}1 \\
1\end{array}$ & 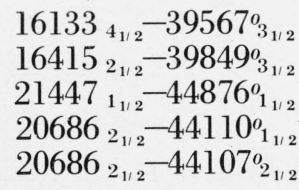 \\
\hline
\end{tabular}


TABLE 3. Classified lines of Tc $\mathrm{I}$

\begin{tabular}{|c|c|c|c|c|}
\hline \multirow{2}{*}{$\begin{array}{c}\text { Wavelength } \\
\text { A }\end{array}$} & \multirow{2}{*}{$\begin{array}{l}\text { Wavenumber } \\
\mathrm{cm}^{-1}\end{array}$} & \multicolumn{2}{|c|}{ Intensity } & \multirow{2}{*}{$\underset{\mathrm{cm}^{-1}}{\text { Energy levels }}$} \\
\hline & & Arc & Spark & \\
\hline $\begin{array}{l}4269.660 \\
4274.971 \\
4275.504 \\
4275.906 \\
4278.895\end{array}$ & $\begin{array}{l}23414.47 \\
23385.39 \\
23382.47 \\
23380.27 \\
23363.94\end{array}$ & $\begin{array}{r}4 \\
800 \\
18 \\
20 \\
800\end{array}$ & $\begin{array}{r}100 \\
2 \\
2 \\
100\end{array}$ & $\begin{array}{l}39185^{o}{ }_{4_{1 / 2}}-62599_{4_{1 / 2}} \\
11890_{0_{1 / 2}}-35276^{o}{ }_{0_{1 / 2}} \\
20523_{2_{1 / 2}}-43905^{o}{ }_{3_{1 / 2}} \\
19791_{4_{1 / 2}}-43172_{5_{1 / 2}}^{o} \\
11578_{1_{1 / 2}}-34942^{o}{ }_{1_{1 / 2}}\end{array}$ \\
\hline $\begin{array}{l}4279.263 \\
4286.353 \\
4286.843 \\
4289.115 \\
4289.563\end{array}$ & $\begin{array}{l}23361.93 \\
23323.29 \\
23320.62 \\
23308.27 \\
23305.84\end{array}$ & $\begin{array}{r}18 \\
4 \\
1 \\
5 \\
20\end{array}$ & $\begin{array}{l}2 \\
1 \\
1 \\
2\end{array}$ & $\begin{array}{l}19903_{3_{1 / 2}}-43265^{\circ}{ }_{4_{1 / 2}} \\
20783_{3_{1 / 2}}-44107^{\circ} o_{2_{1 / 2}} \\
20686_{2_{1 / 2}}-44007^{\circ} o_{1 / 2} \\
19702_{1_{1 / 2}}-43011^{\circ} o_{1 / 2} \\
19791_{4_{1 / 2}}-43097^{o_{1 / 2}}\end{array}$ \\
\hline $\begin{array}{l}4294.351 \\
4297.058 \\
4302.319 \\
4304.848 \\
4305.516\end{array}$ & $\begin{array}{l}23279.85 \\
23265.19 \\
23236.74 \\
23223.09 \\
23219.48\end{array}$ & $\begin{array}{r}80 \\
10000 \\
1 \\
4 \\
15\end{array}$ & $\begin{array}{r}12 \\
1000 \\
1 \\
2\end{array}$ & $\begin{array}{r}16287_{3_{1 / 2}}-39567^{\circ} o_{3_{1 / 2}} \\
0_{2_{1 / 2}}-23265^{\circ} o_{3_{1 / 2}} \\
21450_{4_{1 / 2}}-44687^{\circ} o_{3_{1 / 2}} \\
20783_{3_{1 / 2}}-44007^{\circ} o_{2_{1 / 2}} \\
20686_{2_{1 / 2}}-43905^{\circ} o_{3_{1 / 2}}\end{array}$ \\
\hline $\begin{array}{l}4305.819 \\
4308.615 \\
4309.370 \\
4309.858 \\
4310.222\end{array}$ & $\begin{array}{l}23217.85 \\
23202.78 \\
23198.72 \\
23196.09 \\
23194.13\end{array}$ & $\begin{array}{r}80 \\
15 \\
2 \\
1 \\
60\end{array}$ & $\begin{array}{l}8 \\
2\end{array}$ & $\begin{array}{l}20639_{5_{1 / 2}}-43857^{\circ} 6_{1 / 2} \\
19702_{1_{1 / 2}}-42905^{\circ} o_{2_{1 / 2}} \\
19702_{1_{1 / 2}}-42901^{\circ} o_{1 / 2} \\
23460_{3_{1 / 2}}-46656^{\circ} o_{3_{1 / 2}} \\
19903_{3_{1 / 2}}-43097^{\circ}{ }_{4_{1 / 2}}\end{array}$ \\
\hline $\begin{array}{l}4311.376 \\
4312.504 \\
4314.021 \\
4314.679 \\
4318.061\end{array}$ & $\begin{array}{l}23187.92 \\
23181.86 \\
23173.71 \\
23170.17 \\
23152.03\end{array}$ & $\begin{array}{r}3 \\
10 \\
9 \\
1 \\
6\end{array}$ & $\begin{array}{l}1 \\
1 \\
1\end{array}$ & $\begin{array}{l}20783_{3_{1 / 2}}-43971^{o} 4_{1 / 2} \\
19895_{2_{1 / 2}}-43077^{\circ} 3_{1 / 2} \\
19903_{3_{1 / 2}}-43077^{\circ} 3_{3_{1 / 2}} \\
16428^{o_{2 / 2}}-39598_{2_{1 / 2}}^{2_{1 / 2}} \\
16415_{2_{1 / 2}}-39567^{\circ}{ }_{3_{1 / 2}}\end{array}$ \\
\hline $\begin{array}{l}4318.983 \\
4323.016 \\
4323.356 \\
4323.958 \\
4324.867\end{array}$ & $\begin{array}{l}23147.08 \\
23125.49 \\
23123.67 \\
23120.45 \\
23115.59\end{array}$ & $\begin{array}{r}5 \\
2 \\
80 \\
12 \\
12\end{array}$ & $\begin{array}{l}1 \\
7 \\
2 \\
2\end{array}$ & $\begin{array}{l}24147_{4_{1 / 2}}-47294^{o} 5_{5_{1 / 2}} \\
21450_{4_{1 / 2}}-44576^{o}{ }_{4_{1 / 2}} \\
17282_{3_{1 / 2}}-40406^{o} 3_{1 / 2} \\
17282_{3_{1 / 2}}-40403^{\circ}{ }_{4_{1 / 2}} \\
19895_{2_{1 / 2}}-43011_{2_{1 / 2}}^{o}\end{array}$ \\
\hline $\begin{array}{l}4326.393 \\
4328.778 \\
4330.231 \\
4331.866 \\
4332.452\end{array}$ & $\begin{array}{l}23107.44 \\
23094.71 \\
23086.96 \\
23078.25 \\
23075.13\end{array}$ & $\begin{array}{r}3 \\
60 \\
2 \\
1 \\
150\end{array}$ & $\begin{array}{l}1 \\
6\end{array}$ & $\begin{array}{l}19903_{3_{1 / 2}}-43011_{2_{1 / 2}}^{o} \\
171466_{5_{1 / 2}}-40241^{o} 5_{5_{1 / 2}} \\
21752_{3_{1 / 2}}-44839^{o} 3_{1 / 2} \\
17328_{4_{1 / 2}}-40406^{o} 3_{3_{1 / 2}} \\
17328_{4_{1 / 2}}-40403^{o}{ }_{4_{1 / 2}}\end{array}$ \\
\hline $\begin{array}{l}4335.187 \\
4336.855 \\
4338.248 \\
4341.109 \\
4342.242\end{array}$ & $\begin{array}{l}23060.57 \\
23051.70 \\
23044.30 \\
23029.11 \\
23023.10\end{array}$ & $\begin{array}{c}4 \\
400 c w \\
2 h \\
2 \\
20\end{array}$ & $\begin{array}{c}1 \\
40 \mathrm{cw}\end{array}$ & $\begin{array}{l}21206_{2_{1 / 2}}-44266^{o}{ }_{3_{1 / 2}} \\
11890_{0_{1 / 2}}-34942^{o}{ }_{1_{1 / 2}} \\
16415_{2_{1 / 2}}-39459^{\circ}{ }_{{ }_{2 / 2}} \\
21926_{2_{1 / 2}}-44955^{o}{ }_{{ }_{1 / 2}} \\
20523_{2_{1 / 2}}-43546^{o}{ }_{{ }_{1 / 2}}\end{array}$ \\
\hline $\begin{array}{l}4342.570 \\
4345.442 \\
4350.013 \\
4350.415 \\
4354.823\end{array}$ & $\begin{array}{l}23021.36 \\
23006.15 \\
22981.97 \\
22979.85 \\
22956.59\end{array}$ & $\begin{array}{l}4 \\
10 \\
5 h \\
1 \\
2\end{array}$ & 1 & $\begin{array}{l}20783_{3_{1 / 2}}-43805^{o} 3_{3_{1 / 2}} \\
19895_{2_{1 / 2}}-42901^{o} 1_{1 / 2} \\
19791_{4_{1 / 2}}-42773^{o} o_{5_{1 / 2}} \\
19791_{4_{1 / 2}}-42771^{o} 3_{3_{1 / 2}} \\
21920_{2_{1 / 2}}-44876^{o} 1_{1_{1 / 2}}\end{array}$ \\
\hline $\begin{array}{l}4355.398 \\
4356.093 \\
4358.491 \\
4359.257 \\
4361.959\end{array}$ & $\begin{array}{l}22953.56 \\
22949.90 \\
22937.27 \\
22933.24 \\
22919.03\end{array}$ & $\begin{array}{c}15 w \\
3 \\
400 \\
200 \\
20\end{array}$ & $\begin{array}{r}2 \\
1 \\
40 \\
30 \\
2\end{array}$ & $\begin{array}{l}17282_{3_{1 / 2}}-40236^{0}{ }_{3_{1 / 2}} \\
21926_{2_{1 / 2}}-44876^{0}{ }_{1_{1 / 2}} \\
11578_{1_{1 / 2}}-34515^{0}{ }_{2_{1 / 2}} \\
212044_{4_{1 / 2}}-44138^{0} 5_{5_{1 / 2}} \\
21920_{2_{1 / 2}}-44839^{\circ}{ }_{3_{1 / 2}}\end{array}$ \\
\hline
\end{tabular}


TABLE 3. Classified lines of $\mathrm{Tc} \mathrm{I}$

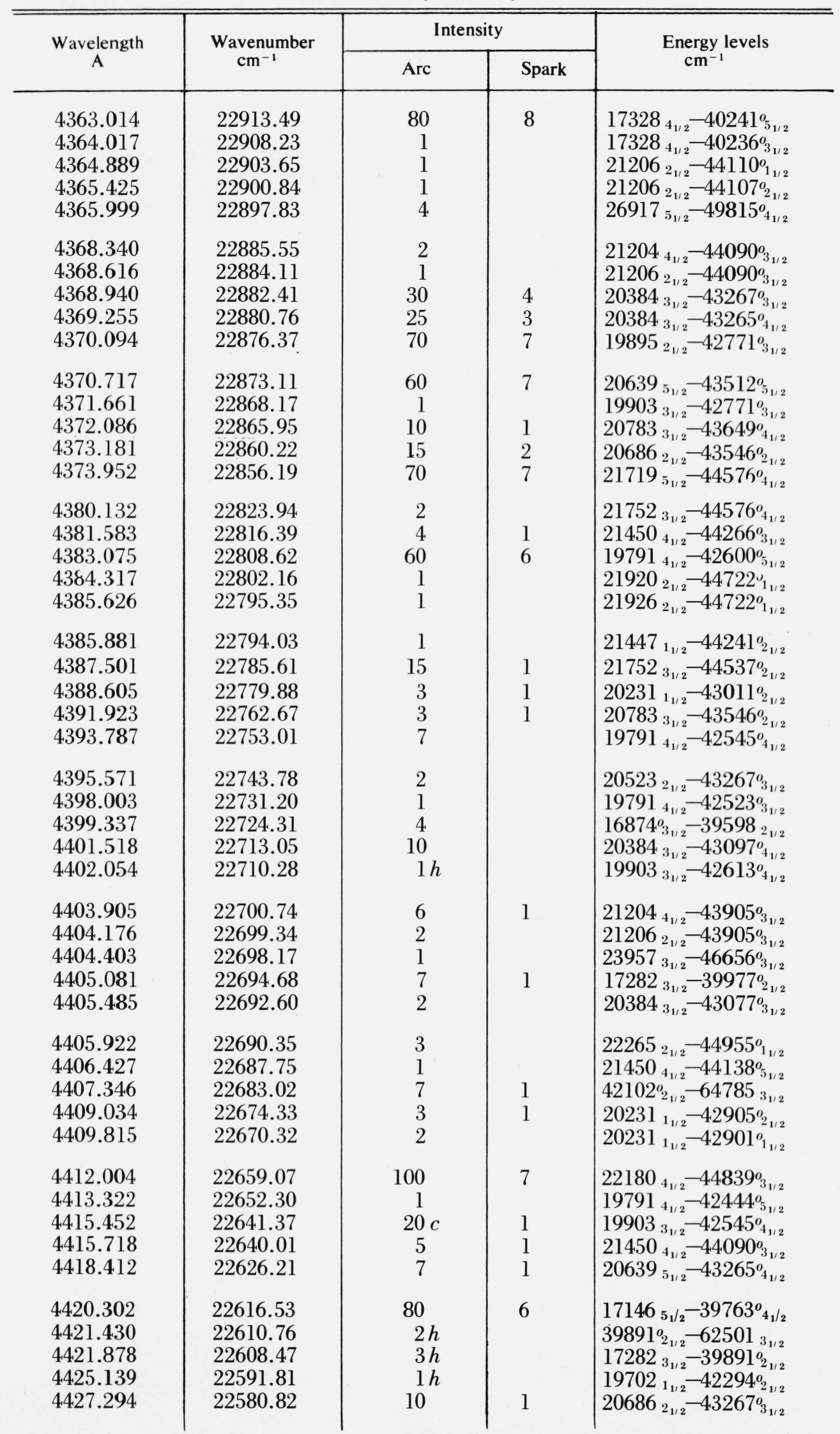


TABLE 3. Classified lines of $\mathrm{Tc} \mathbf{I}$

\begin{tabular}{|c|c|c|c|c|}
\hline \multirow{2}{*}{$\begin{array}{l}\text { Wavelength } \\
\text { A }\end{array}$} & \multirow{2}{*}{$\begin{array}{l}\text { Wavenumber } \\
\mathrm{cm}^{-1}\end{array}$} & \multicolumn{2}{|c|}{ Intensity } & \multirow{2}{*}{$\begin{array}{c}\text { Energy levels } \\
\mathrm{cm}^{-1}\end{array}$} \\
\hline & & Arc & Spark & \\
\hline 4428.702 & 22573.64 & 2 & 1 & $222655_{2,4}-4489^{o}$ \\
\hline 4429.592 & 22569.10 & 1000 & 200 & $10516_{3_{1 / 2}}^{2}-33085^{o} o_{2_{1 / 2}}^{b_{1 / 2}}$ \\
\hline 4431.716 & 22558.28 & 15 & 2 & $20639_{5_{1 / 2}}-431970_{6_{1 / 2}}^{-1 / 2}$ \\
\hline 4432.571 & 22553.93 & 12 & 2 & $20523_{2_{1 / 2}}^{1 / 2}-43077_{3_{1 / 2}}^{0}$ \\
\hline 4433.783 & 22547.77 & $1 h$ & & $19702_{1_{1 / 2}}^{z_{1 / 2}}-42250_{2_{1 / 2}}^{s_{1 / 2}}$ \\
\hline 4436.731 & 22532.79 & 2 & & $20639_{5_{1 / 2}}-43172^{o} \sigma_{5_{1 / 2}}$ \\
\hline 4437.056 & 22531.14 & $1 h$ & & $31414_{3_{1 / 2}}^{0}-53945_{2_{1 / 2}}^{0}$ \\
\hline 4438.999 & 22521.27 & 5 & & $17328_{4_{1 / 2}}-39849^{\circ}{ }_{3_{1 / 2}}$ \\
\hline 4441.418 & 22509.01 & $2 h$ & & $24147_{4_{1 / 2}}-46656^{0_{1 / 2}}$ \\
\hline 4446.502 & 22483.27 & 8 & 2 & $20783_{3_{1 / 2}}-43267_{3_{1 / 2}}^{1 / 2}$ \\
\hline 4447.015 & 22480.68 & $12 c$ & & $17282_{3_{1 / 2}}-39763_{4_{1 / 2}}^{o}$ \\
\hline 4450.790 & 22461.61 & $5 c$ & 1 & $24147_{t_{1 / 2}}-46608_{3_{1 / 2}}^{t_{1 / 2}}$ \\
\hline 4451.433 & 22458.37 & 1 & & $20639_{5_{1 / 2}}-43097^{0_{1 / 2}}$ \\
\hline 4452.078 & 22455.11 & 6 & & $21450_{4_{1 / 2}}^{1 / 2}-43905_{3_{1 / 2}}^{o_{1 / 2}}$ \\
\hline 4452.273 & 22454.13 & 60 & 6 & $19791_{4_{1 / 2}}-42246_{3_{1 / 2}}^{0}$ \\
\hline 4454.142 & 22444.71 & 3 & & $21204_{4_{1 / 2}}-43649^{\circ}{ }_{4_{1 / 2}}$ \\
\hline 4454.811 & 22441.34 & 1 & & $31503^{0_{1 / 2}}-53945_{2_{1 / 2}}^{a_{1 / 2}}$ \\
\hline 4456.621 & 22432.22 & 20 & 3 & $20384_{3_{1 / 2}}-42817^{0} 0_{1 / 2}^{1 / 2}$ \\
\hline 4458.057 & 22425.00 & 3 & & $19791_{4_{1 / 2}}-42217^{\circ}{ }_{4_{1 / 2} / 2}$ \\
\hline 4459.395 & 22418.27 & 10 & 2 & $21719_{5_{1 / 2}}-44138_{5_{1 / 2}}$ \\
\hline 4463.038 & 22399.97 & 20 & 2 & $19702_{1_{12}}-42102^{o}$ \\
\hline 4463.535 & 22397.48 & 1 & & $41529^{\circ}{ }_{3_{1 / 2}}-63926_{2_{1 / 2}}^{2}$ \\
\hline 4463.852 & 22395.89 & 60 & 6 & $22180_{4_{1 / 2}}^{0}-44576_{t_{1 / 2}}^{0}$ \\
\hline 4464.277 & 22393.75 & 15 & 1 & $25058_{3_{1 / 2}}-47452^{o}{ }_{2_{1 / 2}}$ \\
\hline 4464.864 & 22390.81 & 6 & & $19903_{3_{1 / 2}}^{s_{1 / 2}}-42294_{2_{1 / 2}}^{2}$ \\
\hline 4465.090 & 22389.68 & 20 & 2 & $19827_{0_{1 / 2}}-42217^{o}{ }_{t_{1 / 2}}$ \\
\hline 4465.613 & 22387.06 & 80 & 8 & $20384_{3_{1 / 2}}^{0}-42771_{3_{1 / 2}}^{o_{1 / 2}}$ \\
\hline 4466.575 & 22382.23 & 3 & 1 & $20523_{2_{1 / 2}}-42905^{o}{ }_{2 / / 2}^{1 / 2}$ \\
\hline 4467.384 & 22378.18 & 3 & 1 & $20523_{1_{1 / 2}}-42901^{o_{1 / 2}}$ \\
\hline 4468.309 & 22373.55 & $1 h$ & & $22503_{1_{1 / 2}}^{1 / 2}-44876_{1_{1 / 2}}^{1 / 2}$ \\
\hline 4469.173 & 22369.22 & 10 & 1 & $22503_{1_{1 / 2}}-44872_{2_{1 / 2}}^{o}$ \\
\hline 4470.369 & 22363.24 & 1 & & $40236_{3_{1 / 2}}^{0}-62599_{4_{1 / 2}}^{1 / 2}$ \\
\hline 4471.986 & 22355.15 & 3 & & $19895_{2 / 2}-42250_{2_{1 / 2}}^{o_{1 / 2}}$ \\
\hline 4473.662 & 22346.78 & $15 c$ & 2 & $21920_{2_{1 / 2}}^{-1 / 2}-44266_{3_{1 / 2}}^{-1 / 2}$ \\
\hline 4474.506 & 22342.56 & 8 & 1 & $19903_{3_{1 / 2}}-42246_{3_{1 / 2}}^{o_{1 / 2}}$ \\
\hline 4475.003 & 22340.08 & 7 & 1 & $21926_{2_{1 / 2}}-44266^{\circ}{ }_{3_{1 / 2}}$ \\
\hline 4478.673 & 22321.77 & 4 & 1 & $19895_{2_{1 / 2}}^{-1 / 2}-42217^{0}{ }_{1 / 2}^{1 / 2}$ \\
\hline 4480.251 & 22313.91 & 100 & 10 & $20783_{3_{1 / 2}}-43097_{4_{1 / 2}}^{1 / 2}$ \\
\hline 4481.533 & 22307.53 & 1000 & 100 & $21204_{4_{1 / 2}}^{s_{1 / 2}}-43512^{o_{5_{1 / 2}}^{t_{1 / 2}}}$ \\
\hline 4484.351 & 22293.51 & 10 & 1 & $20783_{3_{1 / 2}}-43077_{3_{1 / 2}}$ \\
\hline 4486.050 & 22285.07 & $6 c$ & 1 & $17282_{3_{1 / 2}}-39567_{3_{1 / 2}}$ \\
\hline 4487.061 & 22280.05 & 3000 & 300 & $11063_{2_{1 / 2}}-33343_{1_{1 / 2}} o_{1 / 2}$ \\
\hline 4488.624 & 22272.29 & 3 & & $22265_{2_{1 / 2}}^{-1 / 2}-44537_{2_{1 / 2}}^{1 / 2}$ \\
\hline 4490.128 & 22264.83 & 1 & & $25405_{1_{1 / 2}}^{1 / 2}-47670_{0}^{o} o_{1 / 2}^{1 / 2}$ \\
\hline 4492.735 & 22251.91 & 10 & 1 & $21719_{\tau_{1 / 2}}-43971_{t_{1 / 2}}^{0_{1 / 2}}$ \\
\hline 4493.388 & 22248.68 & $8 h$ & $7 h$ & $23460_{3_{1 / 2}}-45708_{4_{1 / 2}}$ \\
\hline 4495.027 & 22240.56 & 400 & 50 & $19791_{4_{1 / 2}}-42032^{\circ} \tilde{5}_{1 / 2}$ \\
\hline 4495.213 & 22239.64 & 20 & & $17328_{4_{1 / 2}}-39567^{0} o_{1 / 2}^{1 / 2}$ \\
\hline 4497.337 & 22229.14 & $2 w$ & & $20384_{3_{1 / 2}}-42613^{\circ}{ }_{1 / 2}$ \\
\hline 4497.713 & 22227.28 & 5 & & $20783_{3_{1 / 2}}-43011_{2_{1 / 2}}^{o}$ \\
\hline
\end{tabular}


TAB LE 3. Classified lines of $\mathrm{Tc} \mathrm{I}$

\begin{tabular}{|c|c|c|c|c|}
\hline \multirow{2}{*}{$\underset{\text { W }}{\text { Wavelength }}$} & \multirow{2}{*}{$\begin{array}{l}\text { Wavenumber } \\
\mathrm{cm}^{-1}\end{array}$} & \multicolumn{2}{|c|}{ Intensity } & \multirow{2}{*}{$\begin{array}{l}\text { Energy levels } \\
\mathrm{cm}^{-1}\end{array}$} \\
\hline & & Arc & Spark & \\
\hline 4498.529 & 22223.25 & 60 & 8 & $22615_{312}-44839^{\circ}{ }_{312}$ \\
\hline 4498.529 & 22223.25 & 60 & 8 & $25058_{3_{1 / 2}}-47281_{2_{1 / 2}}^{o_{1 / 2}}$ \\
\hline 4499.295 & 22219.47 & $7 h$ & $1 h$ & $20686_{2_{1 / 2}}-42905^{\circ}{ }_{2_{1 / 2}}$ \\
\hline 4501.743 & 22207.38 & 60 & 6 & $19895_{2_{1 / 2}}-42102^{\circ}{ }_{2_{1 / 2}}$ \\
\hline 4503.399 & 22199.22 & 100 & 10 & $21450_{4_{1 / 2}}-43649^{\circ}{ }_{4 / 2}$ \\
\hline 4505.473 & 22189.00 & 2 & & $39849^{o_{312}}-62038_{3_{12}}$ \\
\hline 4505.881 & 22186.99 & 3 & & $21920_{2_{1 / 2}}-44107_{2_{1 / 2}}^{o_{1 / 2}}$ \\
\hline 4506.640 & 22183.25 & 2 & & $21926_{2_{1 / 2}}^{2}-44110^{o}{ }_{1 / 2}^{z_{1 / 2}}$ \\
\hline 4507.177 & 22180.61 & 4 & & $26917_{5_{1 / 2}}-49098^{1 / 2}{ }_{4_{1 / 2}}^{1_{1 / 2}}$ \\
\hline 4507.775 & 22177.67 & 3 & & $20639_{5_{1 / 2}}^{\sigma_{1 / 2}}-42817^{\circ}{ }_{4_{1 / 2}}$ \\
\hline 4508.719 & 22173.03 & 8 & 1 & $20523_{2_{1 / 2}}-42696^{\circ}{ }_{2_{1 / 2}}$ \\
\hline 4511.312 & 22160.28 & $3 h$ & & $20384_{3_{1 / 2}}-42545^{\circ}{ }_{4_{1 / 2}}$ \\
\hline 4512.691 & 22153.51 & 8 & & $27660^{o}{ }_{3_{1 / 2}}-19813_{2_{1 / 2}}$ \\
\hline 4514.019 & 22146.99 & $2 h$ & & $23957_{3_{1 / 2}}-46104_{2_{1 / 2}}^{o_{1 / 2}}$ \\
\hline 4515.978 & 22137.38 & 1000 & 100 & $21719_{5_{1 / 2}}-43857^{\circ} 6_{1 / 2}^{1 / 2}$ \\
\hline 4522.841 & 22103.79 & 10000 & 1000 & $10516_{3_{1 / 2}}-32620_{3_{1 / 2}}$ \\
\hline 4525.760 & 22089.54 & 2 & & $19903_{3_{1 / 2}}-41993_{3_{1 / 2}}^{o_{1 / 2}}$ \\
\hline 4526.321 & 22086.80 & 10 & 2 & $22180_{4_{1 / 2}}-44266_{3_{1 / 2}}^{0}$ \\
\hline 4526.507 & 22085.89 & 8 & 1 & $19702_{1_{1 / 2}}-41788^{o}{ }_{1 / 2}$ \\
\hline 4531.157 & 22063.23 & 30 & 4 & $20231_{1_{1 / 2}}^{1 / 2}-42294^{o}{ }_{21 / 2}^{1 / 2}$ \\
\hline 4531.391 & 22062.09 & 8 & 1 & $21204_{4_{1 / 2}}-43267^{o} o_{3_{1 / 2}}$ \\
\hline 4531.391 & 22062.09 & 8 & 1 & $21450_{4_{1 / 2}}-43512^{o_{5_{1 / 2}}}$ \\
\hline 4531.703 & 22060.57 & 20 & 3 & $21204_{4_{1 / 2}}-43265^{\circ}{ }_{4_{1 / 2}}$ \\
\hline 4534.236 & 22048.25 & 1 & & $22265_{2_{1 / 2}}-44313^{o} o_{1 / 2}^{1 / 2}$ \\
\hline 4537.052 & 22034.56 & 2 & & $22503_{1_{1 / 2}}-44537_{2_{1 / 2}}$ \\
\hline 4537.347 & 22033.13 & 100 & 10 & $20783_{3_{1 / 2}}-42817^{o}{ }_{4_{1 / 2}}$ \\
\hline 4538.556 & 22027.26 & 3 & 1 & $19895_{2_{1 / 2}}-41922_{2_{1 / 2}}^{o}$ \\
\hline 4539.530 & 22022.53 & 2000 & 500 & $11063_{2_{1 / 2}}-33085_{2_{1 / 2}}^{-1 / 2}$ \\
\hline 4541.247 & 22014.21 & 5 & & $42771_{3_{1 / 2}}-64785_{3_{1 / 2}}$ \\
\hline 4542.090 & 22010.12 & 400 & 40 & $20686_{2_{1 / 2}}-42696_{2_{1 / 2}}^{0}$ \\
\hline 4543.900 & 22001.35 & 5 & 1 & $22265_{2}-44266^{\circ}$ \\
\hline 4544.609 & 21997.92 & 3 & & $27940_{2_{1 / 2}}^{o}-49938_{2_{1 / 2}}$ \\
\hline 4545.546 & 21993.39 & 50 & 5 & $19903_{3_{1 / 2}}-41896^{\circ}{ }_{4_{1 / 2}}$ \\
\hline 4546.672 & 21987.94 & 2 & & $20783_{3_{1 / 2}}-42771_{3_{1 / 2}}$ \\
\hline 4549.439 & 21974.57 & 80 & 8 & $20639_{5_{1 / 2}}-42613^{o}{ }_{4_{1 / 2}}$ \\
\hline 4552.204 & 21961.22 & 400 & 40 & $20639_{5_{1 / 2}}-42600^{\circ} \overline{5}_{1_{1 / 2}}$ \\
\hline 4552.854 & 21958.09 & 800 & 80 & $22180_{4_{1 / 2}}-44138_{5_{1^{\prime} / 2}}$ \\
\hline 4557.046 & 21937.89 & 1000 & 100 & $19791_{4_{1 / 2}}-417290_{3_{1 / 2}}$ \\
\hline 4558.711 & 21929.87 & 40 & 4 & $21719_{5_{1 / 2}}-43649^{\circ}{ }_{4_{1 / 2}}$ \\
\hline 4560.205 & 21922.69 & 15 & 2 & $19702_{1_{1 / 2}}-41625^{\circ}{ }_{1_{1 / 2}}$ \\
\hline 4560.421 & 21921.65 & $20 c$ & 2 & $24147_{4_{1 / 2}}-46068^{o}{ }_{4_{1 / 2}}$ \\
\hline 4562.522 & 21911.56 & 8 & 1 & $19791_{4_{1 / 2}}-41703_{4_{1 / 2}}^{o}$ \\
\hline 4562.913 & 21909.68 & 30 & 3 & $20384_{3_{1 / 2}}-42294_{2_{1 / 2}}^{o}$ \\
\hline 4563.741 & 21905.70 & 25 & 2 & $20639_{5_{1 / 2}}-42545^{0}{ }_{4_{1 / 2}}$ \\
\hline 4564.539 & 21901.87 & 2000 & 300 & $11578_{1_{1 / 2}}-33480^{\circ} 0_{1 / 2}$ \\
\hline 4565.436 & 21897.57 & 4 & 1 & $21752_{3_{1 / 2}}-43649^{\circ}{ }_{4_{1 / 2}}$ \\
\hline 4566.421 & 21892.85 & 7 & 1 & $21204_{4_{1 / 2}}-43097_{4_{1 / 2}}$ \\
\hline 4568.135 & 21884.63 & 3 & & $29539_{4_{1 / 2}}-51423_{3_{1 / 2}}^{o}$ \\
\hline 4570.599 & 21872.84 & 15 & & $27940^{\circ} o_{2_{1 / 2}}-49813_{2_{1 / 2}}$ \\
\hline 4570.861 & 21871.58 & 60 & 10 & $20231_{1_{1 / 2}}-42102_{2_{1 / 2}}$ \\
\hline
\end{tabular}


TABLE 3. Classified lines of $\mathrm{Tc} \mathrm{I}$

\begin{tabular}{|c|c|c|c|c|}
\hline \multirow{2}{*}{$\begin{array}{c}\text { Wavelength } \\
\text { A }\end{array}$} & \multirow{2}{*}{$\begin{array}{l}\text { Wavenumber } \\
\mathrm{cm}^{-1}\end{array}$} & \multicolumn{2}{|c|}{ Intensity } & \multirow{2}{*}{$\begin{array}{c}\text { Energy levels } \\
\mathrm{cm}^{-1}\end{array}$} \\
\hline & & Arc & Spark & \\
\hline $\begin{array}{l}4571.453 \\
4572.062 \\
4573.977 \\
4576.521 \\
4576.521\end{array}$ & $\begin{array}{l}21868.75 \\
21865.84 \\
21856.68 \\
21844.53 \\
21844.53\end{array}$ & $\begin{array}{l}2 \\
5 \\
8 \\
2 \\
2\end{array}$ & $\begin{array}{l}1 \\
1\end{array}$ & $\begin{array}{l}198955_{2_{1 / 2}}-41764^{o}{ }_{2_{1 / 2}} \\
20384{ }_{3_{1 / 2}}-42250^{o}{ }_{2_{1 / 2}} \\
251744_{2_{1 / 2}}-47030^{o}{ }_{1_{1 / 2}} \\
27971_{4_{1 / 2}}-49815^{o}{ }_{{ }_{1 / 2}} \\
222655_{2_{1 / 2}}-44110^{o}{ }_{1_{1 / 2}}\end{array}$ \\
\hline $\begin{array}{l}4577.129 \\
4578.447 \\
4579.559 \\
4580.291 \\
4580.613\end{array}$ & $\begin{array}{l}21841.63 \\
21835.34 \\
21830.04 \\
21826.55 \\
21825.02\end{array}$ & $\begin{array}{c}20 \\
1000 \\
3 \\
3 w \\
7\end{array}$ & $\begin{array}{r}3 \\
100\end{array}$ & 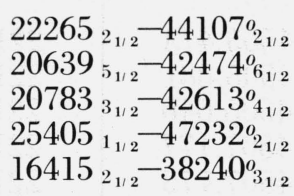 \\
\hline $\begin{array}{l}4582.377 \\
4582.726 \\
4583.246 \\
4583.686 \\
4584.851\end{array}$ & $\begin{array}{l}21816.62 \\
21814.96 \\
21812.48 \\
21810.39 \\
21804.85\end{array}$ & $\begin{array}{r}25 \\
4 \\
1 \\
2 \\
50\end{array}$ & $\begin{array}{l}3 \\
1\end{array}$ & $\begin{array}{l}21450_{4_{1 / 2}}-43267^{0} 0_{3_{1 / 2}} \\
21450_{4_{1 / 2}}-43265^{o}{ }_{4_{1 / 2}} \\
23455_{2_{1 / 2}}^{o_{2}}-45267_{1_{1 / 2}} \\
22503_{1_{1 / 2}}-44313^{o} o_{1_{1 / 2}} \\
20639_{5_{1 / 2}}-42444^{0} o_{5_{1 / 2}}\end{array}$ \\
\hline $\begin{array}{l}4585.884 \\
4586.309 \\
4587.037 \\
4587.628 \\
4588.232\end{array}$ & $\begin{array}{l}21799.93 \\
21797.91 \\
21794.45 \\
21791.65 \\
21788.78\end{array}$ & $\begin{array}{r}150 \\
4 \\
6 \\
4 \\
30\end{array}$ & $\begin{array}{r}15 \\
1 \\
1 \\
1 \\
3\end{array}$ & $\begin{array}{l}19903_{3_{1 / 2}}-41703^{o} o_{4_{1 / 2}} \\
19827^{0_{1 / 2}}-41625^{o}{ }_{1_{1 / 2}} \\
27940^{0} o_{2_{1 / 2}}-49735_{1_{1 / 2}} \\
22180_{4_{1 / 2}}-43971^{o}{ }_{4_{1 / 2}} \\
19791_{4_{1 / 2}}-41580^{o} o_{5_{1 / 2}}\end{array}$ \\
\hline $\begin{array}{l}4588.536 \\
4591.988 \\
4593.346 \\
4594.059 \\
4598.217\end{array}$ & $\begin{array}{l}21787.33 \\
21770.96 \\
21764.52 \\
21761.14 \\
21741.46\end{array}$ & $\begin{array}{r}2 \\
1 \\
1000 \\
100 \\
10\end{array}$ & $\begin{array}{r}2 \\
100 \\
10 \\
1\end{array}$ & 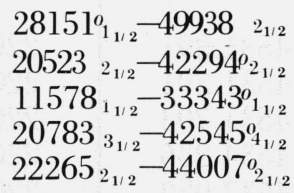 \\
\hline $\begin{array}{l}4601.231 \\
4601.586 \\
4602.408 \\
4602.721 \\
4603.162\end{array}$ & $\begin{array}{l}21727.22 \\
21725.55 \\
21721.67 \\
21720.19 \\
21718.11\end{array}$ & $\begin{array}{r}3 \\
2 \\
30 \\
100 \\
15\end{array}$ & $\begin{array}{r}3 \\
10 \\
2\end{array}$ & $\begin{array}{l}205233_{2_{1 / 2}}-42250^{o}{ }_{2_{1 / 2}} \\
22180_{4_{1 / 2}}-43905^{o}{ }_{3_{1 / 2}} \\
21450_{4_{1 / 2}}-43172^{o} o_{5_{1 / 2}} \\
23265^{o} o_{3_{1 / 2}}-44985_{3_{1 / 2}} \\
20384_{3_{1 / 2}}-42102^{o} o_{2_{1 / 2}}\end{array}$ \\
\hline $\begin{array}{l}4607.170 \\
4608.005 \\
4608.314 \\
4608.833 \\
4609.159\end{array}$ & $\begin{array}{l}21699.22 \\
21695.28 \\
21693.83 \\
21691.39 \\
21689.85\end{array}$ & $\begin{array}{r}3 \\
80 \\
20 \\
8 \\
300 c\end{array}$ & $\begin{array}{c}8 \\
2 \\
1 \\
30 c\end{array}$ & $\begin{array}{l}21206{ }_{2_{1 / 2}}-42905^{o}{ }_{{ }_{1 / 2}} \\
21206{ }_{2_{1 / 2}}-42901^{o}{ }_{1_{1 / 2}} \\
205232_{2_{1 / 2}}-42217^{o} o_{1 / 2} \\
20231_{1_{1 / 2}}-41922^{o} o_{2_{1 / 2}} \\
13252_{2_{1 / 2}}-34942^{o} o_{1_{1 / 2}}\end{array}$ \\
\hline $\begin{array}{l}4611.171 \\
4611.171 \\
4611.386 \\
4611.811 \\
4616.857\end{array}$ & $\begin{array}{l}21680.39 \\
21680.39 \\
21679.38 \\
21677.38 \\
21653.69\end{array}$ & $\begin{array}{r}40 \\
40 \\
2 \\
3 \\
1000\end{array}$ & 100 & 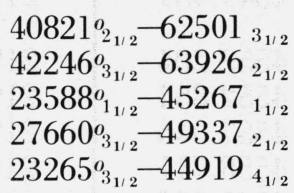 \\
\hline $\begin{array}{l}4617.447 \\
4619.725 \\
4621.129 \\
4621.458 \\
4622.691\end{array}$ & $\begin{array}{l}21650.92 \\
21640.24 \\
21633.67 \\
21632.13 \\
21626.36\end{array}$ & $\begin{array}{c}20 \\
1 \\
30 \\
40 \\
200 c\end{array}$ & $20 c$ & 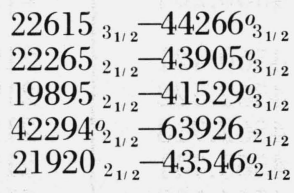 \\
\hline $\begin{array}{l}4622.950 \\
4622.950 \\
4624.122 \\
4624.961 \\
4625.628\end{array}$ & $\begin{array}{l}21625.15 \\
21625.15 \\
21619.67 \\
21615.75 \\
21612.63\end{array}$ & $\begin{array}{r}30 c \\
30 c \\
3 \\
300 \\
5\end{array}$ & $\begin{array}{c}1 c \\
1 c \\
1 \\
30 \\
1\end{array}$ & $\begin{array}{l}27940^{o}{ }_{2_{1 / 2}}-49565_{2_{1 / 2}} \\
22180_{4_{1 / 2}}-43805^{o_{3_{1 / 2}}} \\
21926_{2_{1 / 2}}-43546_{2_{1 / 2}}^{o} \\
23455_{2_{1 / 2}}^{o}-45071_{2_{1 / 2}}^{o_{2}} \\
27517_{2_{1 / 2}}-49130^{o} o_{3_{1 / 2}}\end{array}$ \\
\hline
\end{tabular}


TABLE 3. Classified lines of TC $\mathrm{I}$

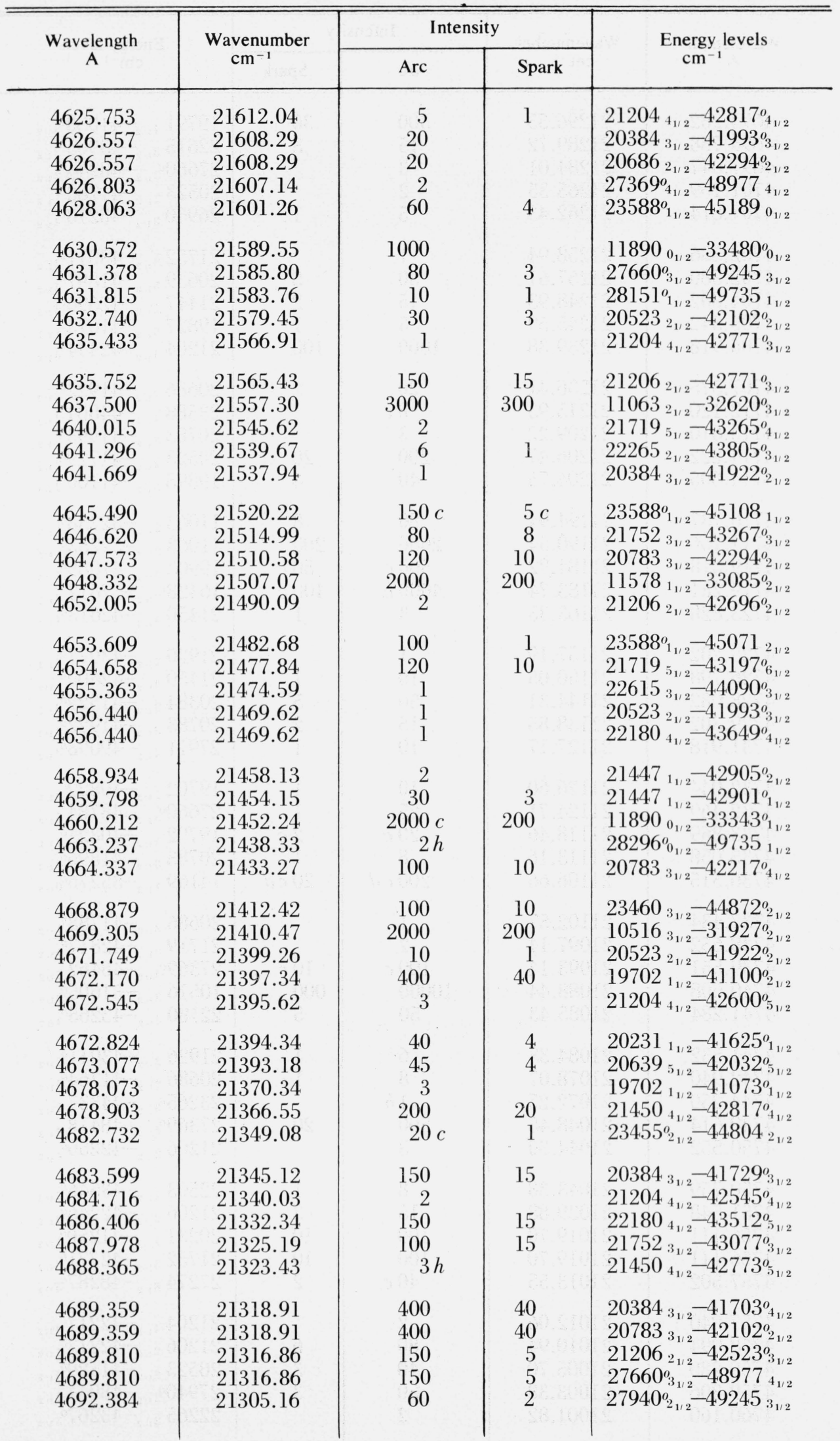


TABLE 3. Classified lines of $\mathrm{Tc} \mathrm{I}$

\begin{tabular}{|c|c|c|c|c|}
\hline \multirow{2}{*}{$\begin{array}{l}\text { Wavelength } \\
\text { A }\end{array}$} & \multirow{2}{*}{$\begin{array}{c}\text { Wavenumber } \\
\mathrm{cm}^{-1}\end{array}$} & \multicolumn{2}{|c|}{ Intensity } & \multirow{2}{*}{$\begin{array}{l}\text { Energy levels } \\
\mathrm{cm}^{-1}\end{array}$} \\
\hline & & Arc & Spark & \\
\hline 4694.282 & 21296.55 & 300 & 30 & $19791_{4}-41088^{o_{3}}$ \\
\hline 4695.788 & 21289.72 & 15 & 3 & $22615_{3_{1 / 2}}-43905^{0}{ }_{3_{1 / 2}}^{s_{1 / 2}}$ \\
\hline 4697.047 & 21284.01 & 3 & & $27660_{3_{1 / 2}}^{0}-48944_{2_{1 / 2}}$ \\
\hline 4701.169 & 21265.35 & 2 & & $20523_{2_{1 / 2}}-41788^{0}{ }_{1_{1 / 2}}$ \\
\hline 4701.814 & 21262.43 & 5 & 1 & $26950_{3_{1 / 2}}^{1 / 2}-48213^{0}{ }_{4_{1 / 2}}^{1 / 2}$ \\
\hline 4702.586 & 21258.94 & 1 & & $21752_{3_{1 / 2}}-43011_{2_{1 / 2}}^{o}$ \\
\hline 4702.880 & 21257.61 & 50 & 5 & $20639_{5_{1 / 2}}-41897^{\circ}{ }_{4_{1 / 2}}^{-1 / 2}$ \\
\hline 4704.802 & 21248.93 & 15 & 2 & $21447_{1_{1 / 2}}-42696_{2_{1 / 2}}^{o}$ \\
\hline 4705.542 & 21245.59 & 5 & 1 & $19827_{0_{1 / 2}}^{1 / 2}-41073_{1_{1 / 2}}^{o_{1 / 2}}$ \\
\hline 4706.918 & 21239.38 & 1000 & 100 & $21204_{4_{1 / 2}}-42444^{\circ} o_{5_{1 / 2}}$ \\
\hline 4707.575 & 21236.41 & 20 & 2 & $20686_{2_{1 / 2}}-41922_{2_{1 / 2}}^{o}$ \\
\hline 4712.120 & 21215.93 & $8 c$ & & $23588^{0}{ }_{1_{1 / 2}}^{-1 / 2}-44804_{2_{1 / 2}}^{-1 / 2}$ \\
\hline 4713.610 & 21209.22 & 3 & & $20783_{3_{1 / 2}}-41993^{0}{ }_{3_{1 / 2}}^{-1 / 2}$ \\
\hline 4714.222 & 21206.47 & 200 & 20 & $20523_{2_{1 / 2}}-41729^{\circ} 0_{3_{1 / 2}}$ \\
\hline 4714.605 & 21204.75 & 40 & 4 & $19895_{2_{1 / 2}}-41100_{2_{1 / 2}}^{\circ}$ \\
\hline 4716.787 & 21194.94 & 80 & 8 & $11063_{2_{1 / 2}}-32258_{1_{1 / 2}}^{o}$ \\
\hline 4717.766 & 21190.54 & 2000 & 200 & $11063_{2_{1 / 2}}-32253^{0}{ }_{1_{1 / 2}}$ \\
\hline 4719.018 & 21184.92 & $500 c$ & $50 c$ & $19903_{3_{1 / 2}}^{-1 / 2}-41088^{0}{ }_{3_{1 / 2}}$ \\
\hline 4719.281 & 21183.74 & $4000 c$ & $400 c$ & $164288_{2_{1 / 2}}-37612_{3_{1 / 2}}$ \\
\hline 4723.828 & 21163.35 & 3 & 1 & $21450_{4_{1 / 2}}-42613_{4_{1 / 2}}^{0}$ \\
\hline 4725.202 & 21157.19 & 1 & & $21920_{2_{1 / 2}}-43077_{3_{1 / 2}}$ \\
\hline 4726.798 & 21150.05 & 10 & 1 & $21450_{4_{1 / 2}}-42600^{\circ} 5_{5_{1 / 2}}$ \\
\hline 4728.082 & 21144.31 & 50 & 5 & $20384_{3_{1 / 2}}-41529^{\circ}{ }_{3_{1 / 2}}$ \\
\hline 4729.302 & 21138.85 & 15 & 2 & $20783_{3_{1 / 2}}-41922_{2_{1 / 2}}^{\circ}$ \\
\hline 4731.918 & 21127.17 & 10 & 1 & $27971_{4_{1 / 2}}-49098^{\circ}{ }_{4_{1 / 2}}$ \\
\hline 4732.044 & 21126.60 & 10 & 1 & $19702_{1_{1 / 2}}-40829^{\circ} 0_{1 / 2}$ \\
\hline 4732.469 & 21124.71 & 25 & 1 & $27660^{\circ} 3_{1_{1 / 2}}-48784_{3_{1 / 2}}$ \\
\hline 4733.869 & 21118.46 & $20 c$ & $2 c$ & $19702_{1_{1 / 2}}-40821_{2_{1 / 2}}$ \\
\hline 4735.058 & 21113.16 & 8 & 1 & $20783_{3_{1 / 2}}-41896^{\circ}{ }_{4 / 2}^{-}$ \\
\hline 4736.515 & 21106.66 & $200 c d$ & $20 c d$ & $14169_{1_{1 / 2}}-35276^{0} 0_{1 / 2}$ \\
\hline 4737.434 & 21102.57 & 1 & & $20686_{2_{1 / 2}}-41788_{1_{1 / 2}}^{o}$ \\
\hline 4738.652 & 21097.14 & 2 & & $21719_{5_{1 / 2}}-42817^{\circ} 0_{4_{1 / 2}}$ \\
\hline 4739.551 & 21093.14 & $100 c$ & $10 c$ & $27369^{\circ}{ }_{4_{1 / 2}}-48462_{3_{1 / 2}}$ \\
\hline 4740.608 & 21088.44 & 10000 & 000 & $10516_{3_{1 / 2}}-31605^{\circ}{ }_{3_{1 / 2}}$ \\
\hline 4741.284 & 21085.43 & 50 & 5 & $22180_{4_{1 / 2}}-43265^{\circ}{ }_{4_{1 / 2}}$ \\
\hline 4741.532 & 21084.33 & 6 & 1 & $21926_{2_{1 / 2}}-43011_{2_{1 / 2}}^{o}$ \\
\hline 4742.940 & 21078.07 & 8 & 1 & $20686_{2_{1 / 2}}-41764_{2_{1 / 2}}^{0}$ \\
\hline 4744.250 & 21072.25 & $1 h$ & & $23265^{o}{ }_{3_{1 / 2}}-44337_{2_{1 / 2}}$ \\
\hline 4749.614 & 21048.45 & 500 & 20 & $27369^{\circ}{ }_{4_{1 / 2}}-48418_{4_{1 / 2}}$ \\
\hline 4750.552 & 21044.30 & 3 & & $21206_{2_{1 / 2}}-42250_{2_{1 / 2}}^{o}$ \\
\hline 4750.759 & 21043.38 & 8 & 1 & $22503_{1_{1 / 2}}-43546_{2_{1 / 2}}^{o}$ \\
\hline 4751.548 & 21039.88 & 15 & 2 & $21206_{2_{1 / 2}}-42246_{3_{1 / 2}}^{0}$ \\
\hline 4756.111 & 21019.70 & 100 & 10 & $20231_{1_{1 / 2}}-41250^{o}{ }_{1_{1 / 2}}$ \\
\hline 4756.111 & 21019.70 & 100 & 10 & $21752_{3_{1 / 2}}-42771_{3_{1 / 2}}^{o}$ \\
\hline 4757.502 & 21013.55 & $40 c$ & 2 & $27274_{6_{1 / 2}}-48287^{0} 0_{5_{1 / 2}}$ \\
\hline 4757.840 & 21012.06 & 2 & & $21204_{4_{1 / 2}}-42217^{\circ}{ }_{4_{1 / 2}}$ \\
\hline 4758.094 & 21010.94 & 80 & 6 & $21206_{2_{1 / 2}}-42217^{0} o_{1 / 2}$ \\
\hline 4759.280 & 21005.70 & 40 & 3 & $20523_{2_{1 / 2}}-415290_{3_{1 / 2}}$ \\
\hline 4759.806 & 21003.38 & 50 & 3 & $27940^{\circ}{ }_{2_{1 / 2}}-48944_{2_{1 / 2}}$ \\
\hline 4760.160 & 21001.82 & 2 & & $22265_{2_{1 / 2}}-43267_{3_{1 / 2}}$ \\
\hline
\end{tabular}


TABLE 3. Classified lines of $\mathrm{Tc} \mathrm{I}$

\begin{tabular}{|c|c|c|c|c|}
\hline \multirow{2}{*}{$\begin{array}{c}\text { Wavelength } \\
\text { A }\end{array}$} & \multirow{2}{*}{$\begin{array}{l}\text { Wavenumber } \\
\mathrm{cm}^{-1}\end{array}$} & \multicolumn{2}{|c|}{ Intensity } & \multirow{2}{*}{$\begin{array}{l}\text { Energy levels } \\
\mathrm{cm}^{-1}\end{array}$} \\
\hline & & Arc & Spark & \\
\hline $\begin{array}{l}4761.968 \\
4762.362 \\
4764.796 \\
4764.987 \\
4764.987\end{array}$ & $\begin{array}{l}20993.85 \\
20992.11 \\
20981.39 \\
20980.55 \\
20980.55\end{array}$ & $\begin{array}{r}40 \\
200 \\
5 \\
3 \\
3\end{array}$ & $\begin{array}{r}3 \\
20\end{array}$ & $\begin{array}{l}21450_{4_{1 / 2}}-42444^{o} o_{5_{1 / 2}} \\
22180_{4_{1 / 2}}-43172_{5_{1 / 2}}^{o} 5_{5_{1 / 2}} \\
21920_{2_{1 / 2}}-42901_{1_{1 / 2}}^{o} \\
20783_{3_{1 / 2}}-41764^{o} o_{1 / 2} \\
43805_{3_{1 / 2}}^{o}-64785_{3_{1 / 2}}^{o}\end{array}$ \\
\hline $\begin{array}{l}4765.381 \\
4766.305 \\
4771.543 \\
4773.237 \\
4773.890\end{array}$ & $\begin{array}{l}20978.81 \\
20974.74 \\
20951.72 \\
20944.28 \\
20941.42\end{array}$ & $\begin{array}{r}8 \\
7 \\
4000 \\
5 \\
200\end{array}$ & $\begin{array}{r}1 \\
1 \\
400 \\
1 \\
20\end{array}$ & $\begin{array}{l}219266_{2_{1 / 2}}-42905^{o} o_{2_{1 / 2}} \\
21926_{2_{1 / 2}}-42901^{o}{ }_{1_{1 / 2}} \\
11063_{2_{1 / 2}}-320144_{2_{1 / 2}}^{o} \\
21752_{3_{1 / 2}}-42696^{o} o_{2_{1 / 2}} \\
20639_{5_{1 / 2}}-41580_{5_{1 / 2}}^{o}\end{array}$ \\
\hline $\begin{array}{l}4776.355 \\
4776.355 \\
4783.925 \\
4784.137 \\
4784.720\end{array}$ & $\begin{array}{l}20930.61 \\
20930.61 \\
20897.49 \\
20896.57 \\
20894.02\end{array}$ & $\begin{array}{r}120 \\
120 \\
200 \\
20 \\
100\end{array}$ & $\begin{array}{r}12 \\
12 \\
20 \\
2 \\
10\end{array}$ & $\begin{array}{l}25174_{2_{1 / 2}}-461044_{2_{1 / 2}}^{o} \\
22615_{3_{1 / 2}}-43546^{o} o_{2_{1 / 2}} \\
10516_{3_{1 / 2}}-314144_{3_{1 / 2}}^{o} \\
21206_{2_{1 / 2}}-42102^{o}{ }_{2_{1 / 2}} \\
21719_{5_{1 / 2}}-42613_{4_{1 / 2}}^{o}\end{array}$ \\
\hline $\begin{array}{l}4785.597 \\
4787.589 \\
4787.758 \\
4790.482 \\
4791.623\end{array}$ & $\begin{array}{l}20890.19 \\
20881.50 \\
20880.76 \\
20868.89 \\
20863.92\end{array}$ & $\begin{array}{r}500 \\
40 \\
100 \\
200 c \\
250\end{array}$ & $\begin{array}{c}50 \\
2 \\
8 \\
20 c \\
20\end{array}$ & $\begin{array}{l}10516_{3_{1 / 2}}-31406^{o} o_{2_{1 / 2}} \\
23957_{3_{1 / 2}}-44839^{\circ}{ }_{3_{1 / 2}} \\
21719_{5_{1 / 2}}-426000^{\circ} \sigma_{5_{1 / 2}} \\
20231_{1_{1 / 2}}-41100_{2_{1 / 2}}^{o} \\
11063_{2_{1 / 2}}-31927^{0} o_{2_{1 / 2}}\end{array}$ \\
\hline $\begin{array}{l}4792.120 \\
4796.176 \\
4796.480 \\
4797.895 \\
4799.978\end{array}$ & $\begin{array}{l}20861.75 \\
20844.11 \\
20842.79 \\
20836.64 \\
20827.60\end{array}$ & $\begin{array}{c}5 \\
3 \\
10 \\
6 h \\
300\end{array}$ & $\begin{array}{r}1 \\
1 \\
30\end{array}$ & $\begin{array}{l}21752_{3_{1 / 2}}-42613^{o} o_{4_{1 / 2}} \\
27940_{2_{1 / 2}}^{o}-48784_{3_{1 / 2}}^{o_{1 / 2}} \\
20686_{2_{1 / 2}}-415290_{3_{1 / 2}}^{o_{1 / 2}} \\
23265^{o} o_{3_{1 / 2}}-44102_{3_{1 / 2}} \\
21204_{4_{1 / 2}}-42032^{o} o_{5_{1 / 2}}\end{array}$ \\
\hline $\begin{array}{l}4800.700 \\
4803.623 \\
4804.777 \\
4805.688 \\
4807.349\end{array}$ & $\begin{array}{l}20824.47 \\
20811.80 \\
20806.80 \\
20802.86 \\
20795.67\end{array}$ & $\begin{array}{c}80 c \\
2 \\
1 h \\
100 \\
8\end{array}$ & $\begin{array}{r}10 \\
1\end{array}$ & $\begin{array}{l}11578_{1_{1 / 2}}-32403^{o} o_{0_{1 / 2}} \\
22265_{2_{1 / 2}}-43077^{0_{1 / 2}} \\
23460_{3_{1 / 2}}-44266^{o}{ }_{3_{1 / 2}} \\
27660_{3_{1 / 2}}^{o}-48462_{3_{1 / 2}}^{o_{1 / 2}} \\
21450_{4_{1 / 2}}-42246^{o_{1 / 2}}\end{array}$ \\
\hline $\begin{array}{l}4807.988 \\
4809.094 \\
4809.416 \\
4810.633 \\
4811.842\end{array}$ & $\begin{array}{l}20792.91 \\
20788.12 \\
20786.73 \\
20781.47 \\
20776.25\end{array}$ & $\begin{array}{r}1 h \\
1 \\
100 \\
2 \\
60\end{array}$ & $\begin{array}{r}10 \\
6\end{array}$ & $\begin{array}{l}28151_{1_{1 / 2}}^{o}-48944_{2_{1 / 2}} \\
21204_{4_{1 / 2}}-41993_{3_{1 / 2}}^{o} \\
21206_{2_{1 / 2}}-41993^{o} 3_{1 / 2} \\
23460_{3_{1 / 2}}-442410_{2_{1 / 2}}^{o} \\
21920_{2_{1 / 2}}-42696_{2_{1 / 2}}^{0}\end{array}$ \\
\hline $\begin{array}{l}4812.630 \\
4813.324 \\
4814.073 \\
4816.062 \\
4816.794\end{array}$ & $\begin{array}{l}20772.85 \\
20769.85 \\
20766.62 \\
20758.05 \\
20754.89\end{array}$ & $\begin{array}{r}3 \\
50 \\
60 \\
30 c \\
500\end{array}$ & $\begin{array}{r}5 \\
6 \\
1 \\
80\end{array}$ & $\begin{array}{l}14169_{1_{1 / 2}}-34942^{o}{ }_{1_{1 / 2}} \\
214477_{1_{1 / 2}}-42217^{\circ}{ }_{4_{1 / 2}} \\
21450_{4_{1 / 2}}-42217^{\circ}{ }_{4_{1 / 2}} \\
27660^{o} o_{1 / 2}-48418_{4_{1 / 2}} \\
21719_{5_{1 / 2}}-42474^{0} o_{6_{1 / 2}}\end{array}$ \\
\hline $\begin{array}{l}4819.024 \\
4820.739 \\
4825.748 \\
4828.698 \\
4831.347\end{array}$ & $\begin{array}{l}20745.29 \\
20737.91 \\
20716.38 \\
20703.73 \\
20692.38\end{array}$ & $\begin{array}{r}1 h \\
10000 \\
20 \\
2 \\
300\end{array}$ & $\begin{array}{r}000 \\
2 \\
30\end{array}$ & $\begin{array}{r}20783_{3_{1 / 2}}-41529^{o} o_{1 / 2} \\
16874_{3_{1 / 2}}^{o}-37612_{3_{1 / 2}} \\
21206_{2_{1 / 2}}-41922^{o} o_{2_{1 / 2}} \\
20384_{3_{1 / 2}}-41088^{o} o_{3_{1 / 2}} \\
2572_{4_{1 / 2}}-23265_{3_{1 / 2}}^{0}\end{array}$ \\
\hline $\begin{array}{l}4834.367 \\
4835.394 \\
4838.034 \\
4839.017 \\
4840.974\end{array}$ & $\begin{array}{l}20679.45 \\
20675.06 \\
20663.77 \\
20659.58 \\
20651.23\end{array}$ & $\begin{array}{r}1000 \\
1000 \\
1 \\
1 \\
20\end{array}$ & $\begin{array}{l}100 \\
100\end{array}$ & $\begin{array}{l}115788_{1_{1 / 2}}-32258^{o}{ }_{1_{1 / 2}} \\
115788_{1_{1 / 2}}-32253^{o}{ }_{1_{1 / 2}} \\
26788_{2_{1 / 2}}-47452^{o}{ }^{o}{ }_{1 / 2} \\
43267^{o_{3 / 2}}-63926^{{ }_{1 / 2}} \\
22615_{3_{1 / 2}}-43267{ }^{\circ}{ }_{3_{1 / 2}}\end{array}$ \\
\hline
\end{tabular}


TABLE 3. Classified lines of $\mathrm{TC} \mathrm{I}$

\begin{tabular}{|c|c|c|c|c|}
\hline \multirow{2}{*}{$\begin{array}{c}\text { Wavelength } \\
\text { A }\end{array}$} & \multirow{2}{*}{$\begin{array}{l}\text { Wavenumber } \\
\mathrm{cm}^{-1}\end{array}$} & \multicolumn{2}{|c|}{ Intensity } & \multirow{2}{*}{$\begin{array}{l}\text { Energy levels } \\
\mathrm{cm}^{-1}\end{array}$} \\
\hline & & Arc & Spark & \\
\hline $\begin{array}{l}4841.364 \\
4842.022 \\
4843.559 \\
4844.326 \\
4848.716\end{array}$ & $\begin{array}{l}20649.56 \\
20646.76 \\
20640.20 \\
20636.94 \\
20618.25\end{array}$ & $\begin{array}{c}100 \\
10 h \\
6 \\
2 \\
3\end{array}$ & $\begin{array}{c}10 \\
1 h \\
1\end{array}$ & $\begin{array}{l}22615_{3_{1 / 2}}-43265^{\circ}{ }_{4_{1 / 2}} \\
23455^{\circ}{ }_{2_{1 / 2}}-44102_{3_{1 / 2}} \\
22265_{2_{1 / 2}}-42905^{\circ} o_{2_{1 / 2}} \\
22180_{4_{1 / 2}}-42817^{\circ}{ }_{4_{1 / 2}} \\
23957_{3_{1 / 2}}-44576^{o}{ }_{4_{1 / 2}}\end{array}$ \\
\hline $\begin{array}{l}4849.608 \\
4850.368 \\
4853.588 \\
4855.343 \\
4857.211\end{array}$ & $\begin{array}{l}20614.46 \\
20611.23 \\
20597.56 \\
20590.11 \\
20582.19\end{array}$ & $\begin{array}{r}40 \\
4 \\
20000 \\
1 \\
100\end{array}$ & $\begin{array}{r}2 \\
2000 \\
10\end{array}$ & $\begin{array}{l}19791_{4_{1 / 2}}-40406^{o}{ }_{3_{1 / 2}} \\
19791_{4_{1 / 2}}-40403^{o}{ }_{4_{1 / 2}} \\
10516_{3_{1 / 2}}-31114^{o} o_{1 / 2} \\
20231_{1_{1 / 2}}-40821^{o} o_{2_{1 / 2}} \\
21450_{4_{1 / 2}-42032^{o} o_{5_{1 / 2}}}\end{array}$ \\
\hline $\begin{array}{l}4858.446 \\
4859.216 \\
4860.424 \\
4861.232 \\
4862.193\end{array}$ & $\begin{array}{l}20576.96 \\
20573.70 \\
20568.59 \\
20565.17 \\
20561.10\end{array}$ & $\begin{array}{c}2 \\
40 c \\
1 \\
20 \\
100 c\end{array}$ & $\begin{array}{l}2 c \\
2 \\
8 c\end{array}$ & $\begin{array}{l}20523_{2_{1 / 2}}-41100_{2_{1 / 2}}^{o} \\
23455_{2_{1 / 2}}^{o}-44028_{1_{1 / 2}} \\
26883_{2_{1 / 2}}-47452^{o}{ }_{2_{1 / 2}} \\
20523_{2_{1 / 2}}-41088^{o}{ }_{3_{1 / 2}} \\
23588_{1_{1 / 2}}^{o}-44149_{0_{1 / 2}}\end{array}$ \\
\hline $\begin{array}{l}4862.931 \\
4866.733 \\
4869.240 \\
4870.772 \\
4873.799\end{array}$ & $\begin{array}{l}20557.98 \\
20541.92 \\
20531.35 \\
20524.89 \\
20512.14\end{array}$ & $\begin{array}{r}40 \\
10000 \\
3 c \\
200 \\
10 c\end{array}$ & $\begin{array}{r}4 \\
1000 \\
20\end{array}$ & $\begin{array}{l}21206_{2_{1 / 2}}-41764^{o} o_{2_{1 / 2}} \\
11063_{2_{1 / 2}}-31605^{o} o_{3_{1 / 2}} \\
23265_{3_{1 / 2}}^{o}-43796_{2_{1 / 2}} \\
21204_{4_{1 / 2}}-41729^{o} o_{3_{1 / 2}} \\
11890_{0_{1 / 2}}-32403_{0_{1 / 2}}^{o}\end{array}$ \\
\hline $\begin{array}{l}4874.768 \\
4875.142 \\
4876.768 \\
4877.016 \\
4877.344\end{array}$ & $\begin{array}{l}20508.06 \\
20506.49 \\
20499.65 \\
20498.61 \\
20497.23\end{array}$ & $\begin{array}{r}30 \\
1 \\
12 c \\
30 \\
15\end{array}$ & $\begin{array}{l}1 \\
1 \\
3 \\
2\end{array}$ & $\begin{array}{l}22503_{1_{1 / 2}}-43011_{2_{1 / 2}}^{o} \\
22265_{2_{1 / 2}}-42771_{3_{1 / 2}}^{o} \\
19903_{3_{1 / 2}}-40403^{o}{ }_{4_{1 / 2}} \\
21204_{4_{1 / 2}}-41703^{\circ}{ }_{4_{1 / 2}} \\
21719_{5_{1 / 2}}-42217^{0} a_{4_{1 / 2}}\end{array}$ \\
\hline $\begin{array}{l}4878.094 \\
4882.586 \\
4885.044 \\
4885.889 \\
4888.695\end{array}$ & $\begin{array}{l}20494.08 \\
20475.23 \\
20464.93 \\
20461.39 \\
20449.64\end{array}$ & $\begin{array}{r}12 \\
8 \\
30 \\
12 \\
100\end{array}$ & $\begin{array}{r}1 \\
1 \\
3 \\
1 \\
10\end{array}$ & $\begin{array}{l}21752_{3_{1 / 2}}-42246^{o}{ }_{3_{1 / 2}} \\
21447_{1_{1 / 2}}-41922^{\circ}{ }_{2_{1 / 2}} \\
21752_{3_{1 / 2}}-42217^{0} o_{4_{1 / 2}} \\
22615_{3_{1 / 2}}-43077^{\circ} 3_{3_{1 / 2}} \\
19791_{4_{1 / 2}}-40241^{o} o_{5_{1 / 2}}\end{array}$ \\
\hline $\begin{array}{l}4889.444 \\
4890.884 \\
4890.884 \\
4891.918 \\
4892.490\end{array}$ & $\begin{array}{l}20446.51 \\
20440.49 \\
20440.49 \\
20436.17 \\
20433.78\end{array}$ & $\begin{array}{r}50 \\
150 c \\
150 c \\
8000 \\
150\end{array}$ & $\begin{array}{c}5 \\
10 c \\
10 c \\
800 \\
15\end{array}$ & $\begin{array}{l}21450_{4_{1 / 2}}-41897^{0}{ }_{4_{1 / 2}} \\
23588^{o}{ }_{1_{1 / 2}}-44028_{1_{1 / 2}} \\
11063_{2_{1 / 2}}-31503^{o}{ }_{1_{1 / 2}} \\
11578_{1_{1 / 2}}-32014^{0} o_{2_{1 / 2}} \\
22180_{4_{1 / 2}}-42613^{0}{ }_{4_{1 / 2}}\end{array}$ \\
\hline $\begin{array}{l}4893.153 \\
4893.651 \\
4895.977 \\
4897.251 \\
4900.046\end{array}$ & $\begin{array}{l}20431.01 \\
20428.93 \\
20419.23 \\
20413.91 \\
20402.27\end{array}$ & $\begin{array}{c}1 h \\
10 c \\
2 \\
8 \\
30\end{array}$ & $\begin{array}{l}1 c \\
1 \\
3\end{array}$ & $\begin{array}{l}22265_{2_{1 / 2}}-42696_{2_{1 / 2}}^{o} \\
24147_{4_{1 / 2}}-44576^{o}{ }_{4_{1 / 2}} \\
21206_{2_{1 / 2}}-41625^{o}{ }_{1_{1 / 2}} \\
20686_{2_{1 / 2}}-41100^{o}{ }_{2_{1 / 2}} \\
20686_{2_{1 / 2}}-41088^{o}{ }_{3_{1 / 2}}\end{array}$ \\
\hline $\begin{array}{l}4900.955 \\
4902.085 \\
4903.747 \\
4906.410 \\
4906.791\end{array}$ & $\begin{array}{l}20398.49 \\
20393.78 \\
20386.87 \\
20375.81 \\
20374.23\end{array}$ & $\begin{array}{r}10 \\
1 \\
2 \\
50 \\
10\end{array}$ & $\begin{array}{l}5 \\
1\end{array}$ & $\begin{array}{l}22503_{1_{1 / 2}}-42901^{o}{ }_{1_{1 / 2}} \\
19702_{1_{1 / 2}}-40096^{o}{ }_{1_{1 / 2}} \\
20686_{2_{1 / 2}}-41073^{\circ} o_{1_{1 / 2}} \\
21204_{4_{1 / 2}}-41580^{\circ} o_{5_{1 / 2}} \\
21920_{2_{1 / 2}}-42944_{2_{1 / 2}}^{o}\end{array}$ \\
\hline $\begin{array}{l}4908.509 \\
4909.053 \\
4909.566 \\
4912.422 \\
4913.020\end{array}$ & $\begin{array}{l}20367.09 \\
20364.84 \\
20362.71 \\
20350.87 \\
20348.39\end{array}$ & $\begin{array}{r}1000 \\
5 \\
2000 \\
5 c \\
500\end{array}$ & $\begin{array}{r}100 \\
1 \\
200 \\
50\end{array}$ & $\begin{array}{l}11890_{0_{1 / 2}}-32258^{o} o_{1_{1 / 2}} \\
22180_{4_{1 / 2}}-42545^{\circ} o_{4_{1 / 2}} \\
11890_{0_{1 / 2}}-32253^{o}{ }_{1_{1 / 2}} \\
11063_{2_{1 / 2}}-314144^{o} 3_{1 / 2} \\
11578_{1_{1 / 2}}-31927^{\circ} o_{2_{1 / 2}}\end{array}$ \\
\hline
\end{tabular}


TABLE 3. Classified lines of TC I

\begin{tabular}{|c|c|c|c|c|}
\hline \multirow{2}{*}{$\begin{array}{c}\text { Wavelength } \\
\text { A }\end{array}$} & \multirow{2}{*}{$\begin{array}{l}\text { Wavenumber } \\
\mathrm{cm}^{-1}\end{array}$} & \multicolumn{2}{|c|}{ Intensity } & \multirow{2}{*}{$\begin{array}{l}\text { Energy levels } \\
\mathrm{cm}^{-1}\end{array}$} \\
\hline & & Arc & Spark & \\
\hline $\begin{array}{l}4914.166 \\
4914.699 \\
4918.917 \\
4918.917 \\
4919.229\end{array}$ & $\begin{array}{l}20343.65 \\
20341.44 \\
20324.00 \\
20324.00 \\
20322.71\end{array}$ & $\begin{array}{c}10 \\
150 c \\
3 \\
3 \\
5\end{array}$ & $\begin{array}{c}1 \\
15 c\end{array}$ & $\begin{array}{l}11063_{2_{1 / 2}}-31406^{o} o_{2_{1 / 2}} \\
23455_{2_{2_{1 / 2}}}^{o}-43796_{2_{1 / 2}} \\
21204_{4_{1 / 2}}-41529^{o} o_{3_{1 / 2}} \\
21926_{2_{1 / 2}}-42250^{o} o_{2_{1 / 2}} \\
21206_{2_{1 / 2}}-41529^{o}{ }_{3_{1 / 2}}\end{array}$ \\
\hline $\begin{array}{l}4920.668 \\
4921.646 \\
4923.601 \\
4925.412 \\
4927.031\end{array}$ & $\begin{array}{l}20316.77 \\
20312.73 \\
20304.67 \\
20297.20 \\
20290.53\end{array}$ & $\begin{array}{c}200<l \\
20 \\
300 \\
2 \\
5\end{array}$ & $\begin{array}{c}20 c \\
2 \\
3 \\
1\end{array}$ & $\begin{array}{l}21447_{1_{1 / 2}}-41764^{o} o_{2_{1 / 2}} \\
21719_{5_{1 / 2}}-42032^{o} \sigma_{5_{1 / 2}} \\
207833_{3_{1 / 2}}-41088^{o} o_{3_{1 / 2}} \\
21920_{2_{1 / 2}}-42217^{\circ} o_{1 / 2} \\
21926_{2_{1 / 2}}-42217^{\circ} o_{1_{1 / 2}}\end{array}$ \\
\hline $\begin{array}{l}4928.676 \\
4929.749 \\
4930.360 \\
4930.904 \\
4933.430\end{array}$ & $\begin{array}{l}20283.76 \\
20279.34 \\
20276.83 \\
20274.59 \\
20264.21\end{array}$ & $\begin{array}{r}3 \\
25 \\
20 \\
15 \\
10\end{array}$ & $\begin{array}{l}2 \\
2\end{array}$ & $\begin{array}{l}23957_{3_{1 / 2}}-442411^{o}{ }_{2_{1 / 2}} \\
21450_{4_{1 / 2}}-417290_{3_{1 / 2}} \\
29539_{4_{1 / 2}}-49815^{\circ}{ }_{4_{1 / 2}} \\
19702_{1_{1 / 2}}-39977^{\circ} o_{2_{1 / 2}} \\
22180_{4_{1 / 2}}-424444_{5_{1 / 2}}^{o}\end{array}$ \\
\hline $\begin{array}{l}4933.798 \\
4936.132 \\
4939.112 \\
4947.078 \\
4948.064\end{array}$ & $\begin{array}{l}20262.70 \\
20253.12 \\
20240.90 \\
20208.31 \\
20204.28\end{array}$ & $\begin{array}{l}25 c \\
40 \\
2 \\
50 c \\
400\end{array}$ & $\begin{array}{l}2 \\
3 \\
\\
5 c \\
40\end{array}$ & $\begin{array}{l}14679_{0_{1 / 2}}-34942^{o}{ }_{1_{1 / 2}} \\
21450_{4_{1 / 2}}-41703^{o}{ }_{4_{1 / 2}} \\
21752_{3_{1 / 2}}-41993^{o} o_{3_{1 / 2}} \\
23588^{o}{ }_{1_{1 / 2}}-43796_{2_{1 / 2}} \\
3250_{3_{1 / 2}}-23455^{o}{ }_{2_{1 / 2}}\end{array}$ \\
\hline $\begin{array}{l}4948.839 \\
4950.743 \\
4953.351 \\
4954.508 \\
4954.713\end{array}$ & $\begin{array}{l}20201.12 \\
20193.35 \\
20182.72 \\
20178.00 \\
20177.17\end{array}$ & $\begin{array}{l}20 \\
2 \\
1 h \\
2 h \\
10\end{array}$ & 1 & $\begin{array}{l}19895_{2_{1 / 2}}-40096^{o}{ }_{1_{1 / 2}} \\
22503_{1_{1 / 2}}-42696^{\circ} o_{2_{1 / 2}} \\
21920_{2_{1 / 2}}-42102^{o} o_{2_{1 / 2}} \\
21447_{1_{1 / 2}}-41625^{o} o_{1 / 2} \\
21719_{5_{1 / 2}}-41897^{\circ} o_{4_{1 / 2}}\end{array}$ \\
\hline $\begin{array}{l}4954.985 \\
4956.337 \\
4959.942 \\
4961.532 \\
4962.654\end{array}$ & $\begin{array}{l}20176.06 \\
20170.56 \\
20155.90 \\
20149.44 \\
20144.88\end{array}$ & $\begin{array}{r}20 \\
40 \\
4 \\
1 \\
10\end{array}$ & $\begin{array}{l}2 \\
4\end{array}$ & $\begin{array}{l}219266_{2_{1 / 2}}-42102^{o}{ }_{2_{1 / 2}} \\
21752_{3_{1 / 2}}-41922^{o} o_{1 / 2} \\
226153_{1 / 2}-42771_{3_{1 / 2}}^{o} \\
239573_{1 / 2}-44107_{2_{1 / 2}}^{o} \\
21752_{3_{1 / 2}}-41896^{o} o_{1 / 2}\end{array}$ \\
\hline $\begin{array}{l}4966.255 \\
4966.255 \\
4967.200 \\
4968.854 \\
4976.340\end{array}$ & $\begin{array}{l}20130.28 \\
20130.28 \\
20126.45 \\
20119.75 \\
20089.48\end{array}$ & $\begin{array}{l}10 \\
10 \\
60 \mathrm{cw} \\
5 c \\
5000\end{array}$ & $\begin{array}{r}1 \\
1 \\
6 \\
500\end{array}$ & $\begin{array}{l}21450_{4_{1 / 2}}-41580_{5_{1 / 2}}^{o} \\
39185^{\circ}{ }_{4_{1 / 2}}-59315_{5_{1 / 2}} \\
23265^{o}{ }_{3_{1 / 2}}-43391_{3_{1 / 2}} \\
24147_{4_{1 / 2}}-44266_{3_{1 / 2}}^{o} \\
17522_{4_{1 / 2}}^{o}-37612_{3_{1 / 2}}\end{array}$ \\
\hline $\begin{array}{l}4978.569 \\
4979.028 \\
4980.214 \\
4980.448 \\
4991.346\end{array}$ & $\begin{array}{l}20080.49 \\
20078.64 \\
20073.85 \\
20072.91 \\
20029.08\end{array}$ & $\begin{array}{r}20 \\
8 \\
3 \\
50 \\
1 h\end{array}$ & $\begin{array}{l}2 \\
1 \\
4\end{array}$ & $\begin{array}{l}226153_{1 / 2}-42696_{2_{1 / 2}}^{o} \\
21450_{4_{1 / 2}}-41529^{o} o_{1 / 2} \\
19903_{3_{1 / 2}}-39977_{2_{1 / 2}}^{\circ} \\
219200_{2_{1 / 2}}-41993^{\circ} 3_{1 / 2} \\
22265_{2_{1 / 2}}-42294_{2_{1 / 2}}^{o}\end{array}$ \\
\hline $\begin{array}{l}4993.978 \\
4994.997 \\
4999.120 \\
5000.831 \\
5002.674\end{array}$ & $\begin{array}{l}20018.53 \\
20014.44 \\
19997.94 \\
19991.10 \\
19983.73\end{array}$ & $\begin{array}{l}80 c \\
400 \\
30 \\
50 c \\
200\end{array}$ & $\begin{array}{r}40 \\
2 \\
3 \\
20\end{array}$ & $\begin{array}{r}20384_{3_{1 / 2}}-40403^{o}{ }_{4_{1 / 2}} \\
3250_{3_{1 / 2}}-23265^{o}{ }_{3_{1 / 2}} \\
22615_{3_{1 / 2}}-42613^{\circ}{ }_{4_{1 / 2}} \\
24147_{4_{1 / 2}}-44138^{o} 5_{5_{1 / 2}} \\
21719_{5_{1 / 2}}-41703^{o}{ }_{4_{1 / 2}}\end{array}$ \\
\hline $\begin{array}{l}5003.405 \\
5005.737 \\
5010.766 \\
5014.517 \\
5017.324\end{array}$ & $\begin{array}{l}19980.81 \\
19971.50 \\
19951.46 \\
19936.53 \\
19925.38\end{array}$ & $\begin{array}{l}2 \\
100 \\
30 \\
200 \mathrm{cw} \\
1 \mathrm{~h}\end{array}$ & $\begin{array}{c}5 \\
2 \\
20 \mathrm{cw}\end{array}$ & $\begin{array}{l}222655_{2_{1 / 2}}-42246^{o} o_{3_{1 / 2}} \\
19791_{4_{1 / 2}}-39763^{o}{ }_{4_{1 / 2}} \\
21752_{3_{1 / 2}}-41703^{o}{ }_{4_{1 / 2}} \\
23455^{o} o_{2_{1 / 2}}-43391_{3_{1 / 2}} \\
11578_{1_{1 / 2}}-31503^{o}{ }_{1_{1 / 2}}\end{array}$ \\
\hline
\end{tabular}


TABLE 3. Classified lines of Tc I

\begin{tabular}{|c|c|c|c|c|}
\hline \multirow{2}{*}{$\begin{array}{c}\text { Wavelength } \\
\text { A }\end{array}$} & \multirow{2}{*}{$\begin{array}{l}\text { Wavenumber } \\
\mathrm{cm}^{-1}\end{array}$} & \multicolumn{2}{|c|}{ Intensity } & \multirow{2}{*}{$\begin{array}{l}\text { Energy levels } \\
\mathrm{cm}^{-1}\end{array}$} \\
\hline & & Arc & Spark & \\
\hline $\begin{array}{l}5026.793 \\
5027.894 \\
5031.663 \\
5032.107 \\
5032.446\end{array}$ & $\begin{array}{l}19887.85 \\
19883.49 \\
19868.60 \\
19866.85 \\
19865.51\end{array}$ & $\begin{array}{r}300 \\
150 \\
4 \\
10 \\
80\end{array}$ & $\begin{array}{r}30 \\
15 \\
1 \\
8\end{array}$ & 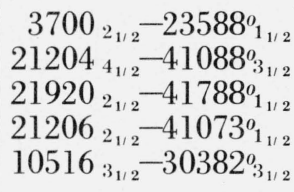 \\
\hline $\begin{array}{l}5033.613 \\
5035.760 \\
5036.002 \\
5036.833 \\
5039.572\end{array}$ & $\begin{array}{l}19860.90 \\
19852.44 \\
19851.48 \\
19848.21 \\
19837.42\end{array}$ & $\begin{array}{r}5 \\
3 \\
4 \\
5 \\
10\end{array}$ & $\begin{array}{l}1 \\
1\end{array}$ & $\begin{array}{l}21719_{5_{1 / 2}}-41580^{o}{ }_{5_{1 / 2}} \\
22180{ }_{4_{1 / 2}}-42032^{o} 5_{5_{1 / 2}} \\
20384_{3_{1 / 2}}-40236^{o}{ }_{3_{1 / 2}} \\
27971_{4_{1 / 2}}-47819^{o}{ }_{{ }_{1 / 2}} \\
22265{ }_{{ }_{1_{1 / 2}}}-42102^{o} o_{{ }_{1 / 2}}\end{array}$ \\
\hline $\begin{array}{l}5041.905 \\
5043.699 \\
5045.580 \\
5046.622 \\
5051.303\end{array}$ & $\begin{array}{l}19828.24 \\
19821.19 \\
19813.80 \\
19809.71 \\
19791.35\end{array}$ & $\begin{array}{l}1 h \\
2 \\
2 h \\
3 h \\
40\end{array}$ & 4 & $\begin{array}{l}11578_{1_{1 / 2}}-31406^{o}{ }_{2_{1 / 2}} \\
42217^{0}{ }_{4_{1 / 2}}-62038_{3_{1 / 2}} \\
25058_{3_{1 / 2}}-44872^{o} 2_{1 / 2} \\
21920_{2_{1 / 2}}-41729^{o} o_{3_{1 / 2}} \\
22503_{1_{1 / 2}}-42294_{2_{1 / 2}}^{o}\end{array}$ \\
\hline $\begin{array}{l}5055.274 \\
5058.331 \\
5060.686 \\
5062.136 \\
5067.632\end{array}$ & $\begin{array}{l}19775.80 \\
19763.85 \\
19754.65 \\
19749.00 \\
19727.58\end{array}$ & $\begin{array}{r}300 \\
60 \\
500 \\
2 \\
1\end{array}$ & $\begin{array}{r}30 \\
6 \\
50\end{array}$ & $\begin{array}{r}19791_{4_{1 / 2}}-39567^{0} 0_{3_{1 / 2}} \\
20639_{5_{1 / 2}}-40403^{0}{ }_{4_{1 / 2}} \\
3700_{2_{1 / 2}}-23455^{0} 0_{2_{1 / 2}} \\
19827_{0_{1 / 2}}-39576^{0} 0_{1_{1 / 2}} \\
22265_{2_{1 / 2}}-41993^{0} o_{3_{1 / 2}}\end{array}$ \\
\hline $\begin{array}{l}5069.549 \\
5070.378 \\
5074.051 \\
5075.184 \\
5079.561\end{array}$ & $\begin{array}{l}19720.12 \\
19716.89 \\
19702.62 \\
19698.22 \\
19681.25\end{array}$ & $\begin{array}{r}50 \\
4 \\
3 \\
10 \\
2\end{array}$ & 5 & $\begin{array}{l}20686_{2_{1 / 2}}-40406^{o}{ }_{3_{1 / 2}} \\
22180_{4_{1 / 2}}-41897^{0}{ }^{0}{ }_{1 / 2} \\
251744_{2_{1 / 2}}-44876^{o}{ }_{1_{1 / 2}} \\
251744_{2_{1 / 2}}-44872^{o} o_{2_{1 / 2}} \\
19895_{2_{1 / 2}}-39576^{0}{ }_{1_{1 / 2}}\end{array}$ \\
\hline $\begin{array}{l}5080.268 \\
5081.860 \\
5083.969 \\
5085.611 \\
5086.922\end{array}$ & $\begin{array}{l}19678.51 \\
19672.35 \\
19664.19 \\
19657.84 \\
19652.77\end{array}$ & $\begin{array}{c}1 h \\
3 \\
30 \\
2 \\
40\end{array}$ & $\begin{array}{l}3 \\
4\end{array}$ & 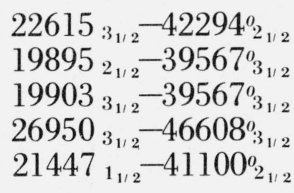 \\
\hline $\begin{array}{l}5088.200 \\
5090.743 \\
5093.182 \\
5095.575 \\
5096.280\end{array}$ & $\begin{array}{l}19647.84 \\
19638.02 \\
19628.62 \\
19619.40 \\
19616.68\end{array}$ & $\begin{array}{r}1 \\
80 \\
10 \\
2 \\
5000\end{array}$ & $\begin{array}{r}8 \\
1 \\
500\end{array}$ & 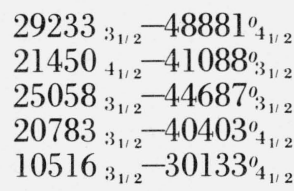 \\
\hline $\begin{array}{l}5100.033 \\
5100.327 \\
5102.525 \\
5103.240 \\
5103.531\end{array}$ & $\begin{array}{l}19602.25 \\
19601.12 \\
19592.68 \\
19589.93 \\
19588.81\end{array}$ & $\begin{array}{c}6 \\
3 \\
30 \\
200 c \\
10\end{array}$ & $\begin{array}{c}1 \\
1 \\
3 \\
20 c \\
1\end{array}$ & $\begin{array}{l}20639_{5_{1 / 2}}-40241^{o}{ }_{5_{1 / 2}} \\
22615_{3_{1 / 2}}-42217^{0}{ }_{i_{1 / 2}} \\
20384_{3_{1 / 2}}-39977^{0}{ }_{a_{1 / 2}} \\
23265^{\circ}{ }_{3_{1 / 2}}-42855_{4_{1 / 2}} \\
23957_{3_{1 / 2}}-43546^{0}{ }_{{ }_{1 / 2}}\end{array}$ \\
\hline $\begin{array}{l}5104.321 \\
5107.581 \\
5109.079 \\
5109.809 \\
5111.113\end{array}$ & $\begin{array}{l}19585.78 \\
19573.28 \\
19567.54 \\
19564.75 \\
19559.76\end{array}$ & $\begin{array}{r}500 \\
8 \\
4 \\
200 \\
1\end{array}$ & $\begin{array}{r}50 \\
1 \\
1 \\
20\end{array}$ & 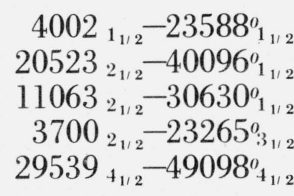 \\
\hline $\begin{array}{l}5112.019 \\
5113.370 \\
5113.686 \\
5120.605 \\
5122.180\end{array}$ & $\begin{array}{l}19556.29 \\
19551.12 \\
19549.91 \\
19523.50 \\
19517.49\end{array}$ & $\begin{array}{r}3 \\
5 \\
30 \\
100 \\
20\end{array}$ & $\begin{array}{r}3 \\
10 \\
2\end{array}$ & $\begin{array}{l}19903_{3_{1 / 2}}-39459^{o}{ }_{2_{1 / 2}} \\
23460_{3_{1 / 2}}-43011^{o}{ }_{2_{1 / 2}} \\
22180_{4_{1 / 2}}-41729^{o}{ }_{3_{1 / 2}} \\
22180_{4_{1 / 2}}-41703^{0}{ }_{4_{1 / 2}} \\
25058_{3_{1 / 2}}-44576^{o}{ }_{4_{1 / 2}}\end{array}$ \\
\hline
\end{tabular}


TABLE 3. Classified lines of $\mathrm{TC} \mathrm{I}$

\begin{tabular}{|c|c|c|c|c|}
\hline \multirow{2}{*}{$\begin{array}{c}\text { Wavelength } \\
\text { A }\end{array}$} & \multirow{2}{*}{$\begin{array}{l}\text { Wavenumber } \\
\mathrm{cm}^{-1}\end{array}$} & \multicolumn{2}{|c|}{ Intensity } & \multirow{2}{*}{$\begin{array}{l}\text { Energy levels } \\
\mathrm{cm}^{-1}\end{array}$} \\
\hline & & Arc & Spark & \\
\hline 5122.739 & 19515.36 & 6 & 1 & $20783_{3_{1 / 2}}-40299^{\circ}{ }_{2_{1 / 2}}$ \\
\hline 5125.158 & 19506.15 & $6 c$ & $1 c$ & $15770_{1_{1 / 2}}-35276_{0_{1^{\prime} / 2}}$ \\
\hline 5130.260 & 19486.76 & $1 h$ & & $22615_{3_{1 / 2}}-42102_{2_{1 / 2}}^{0}$ \\
\hline 5135.789 & 19465.78 & 6 & 1 & $11063_{2_{1 / 2}}-305280_{2_{1 / 2}}^{-1 / 2}$ \\
\hline 5136.156 & 19464.39 & 40 & 4 & $22265_{2_{1 / 2}}-417290_{3_{1 / 2}}$ \\
\hline 5138.886 & 19454.05 & 3 & & $20523_{2_{1 / 2}}-39977_{2_{1 / 2}}$ \\
\hline 5139.262 & 19452.62 & 500 & 50 & $4002_{1_{1 / 2}}-23455_{2_{1 / 2}}^{\circ}$ \\
\hline 5141.227 & 19445.19 & 1 & & $26950_{3_{1 / 2}}-46396_{3_{1 / 2}}^{0}$ \\
\hline 5147.997 & 19419.62 & 4 & & $22503_{1_{1 / 2}}-41922^{\circ}{ }_{2_{1 / 2}}$ \\
\hline 5150.630 & 19409.69 & 500 & 50 & $4178_{0_{1 / 2}}-23588_{1_{1 / 2}}$ \\
\hline 5157.991 & 19381.99 & 10 & 1 & $21447_{1_{1 / 2}}-40829^{\circ} 0_{1 / 2}$ \\
\hline 5158.858 & 19378.73 & $30 c$ & $3 c$ & $20384_{3_{1 / 2}}-39763_{4_{1 / 2}}^{\circ}$ \\
\hline 5160.130 & 19373.96 & 1 & & $28839_{5_{1 / 2}}-48213_{4_{1 / 2}}^{o}$ \\
\hline 5161.810 & 19367.65 & 2000 & 200 & $13252_{2_{1 / 2}}-32620_{3_{1 / 2}}^{o}$ \\
\hline 5161.810 & 19367.65 & 2000 & 200 & $205232_{2_{1 / 2}}-398910_{2_{1 / 2}}$ \\
\hline 5162.410 & 19365.40 & $5 h$ & & $24147_{4_{1 / 2}}-43512_{5_{1 / 2}}^{o}$ \\
\hline 5167.038 & 19348.05 & 1 & & $21752_{3_{1 / 2}}-41100^{\circ} o_{2_{1 / 2}}$ \\
\hline 5167.774 & 19345.30 & 8 & 1 & $20231_{1_{1 / 2}}-39576_{1_{1 / 2}}^{o}$ \\
\hline 5168.470 & 19342.69 & 2 & & $29539_{4_{1 / 2}}-48881^{\circ}{ }_{4_{1 / 2}}$ \\
\hline 5174.812 & 19318.99 & 2000 & 200 & $11063_{2_{1 / 2}}-30382_{3_{1 / 2}}$ \\
\hline 5177.018 & 19310.76 & 2 & & $14169_{1_{1 / 2}}-33480^{\circ} o_{0_{1 / 2}}$ \\
\hline 5177.387 & 19309.38 & 3 & & $23957_{3_{1 / 2}}-43267^{\circ} 3_{3_{1 / 2}}$ \\
\hline 5177.746 & 19308.04 & $10 c$ & & $30630^{o} o_{1_{1 / 2}}-49938_{2_{1 / 2}}$ \\
\hline 5178.104 & 19306.71 & 10 & & $22615_{3_{1 / 2}}-41922^{o}{ }_{1 / 2}$ \\
\hline 5181.930 & 19292.45 & $2 h s$ & & $24564_{5_{1 / 2}}-43857^{\circ} \sigma_{1 / 2}$ \\
\hline 5183.999 & 19284.75 & 50 & 2 & $305288_{2_{1 / 2}}-49813_{2_{1 / 2}}$ \\
\hline 5184.994 & 19281.05 & 10 & 1 & $22615_{3_{1 / 2}}-41896^{o}{ }_{4_{1 / 2}}$ \\
\hline 5189.687 & 19263.62 & 40 & 4 & $222655_{2_{1 / 2}}-41529^{o}{ }_{3_{1 / 2}}$ \\
\hline 5202.152 & 19217.46 & $10 \mathrm{cw}$ & & $15298_{3_{1 / 2}}-34515^{0} o_{2_{1 / 2}}$ \\
\hline 5206.862 & 19200.07 & 10 & & $21206_{2_{1 / 2}}-40406_{3_{1 / 2}}^{0}$ \\
\hline 5207.358 & 19198.25 & 4 & & $21204_{4_{1 / 2}}-40403^{o}{ }_{4_{1 / 2}}$ \\
\hline 5208.623 & 19193.58 & 25 & & $20783_{3_{1 / 2}}-39977_{2_{1 / 2}}$ \\
\hline 5211.262 & 19183.86 & 6 & & $30382_{3_{1 / 2}}^{o}-49565_{2_{1 / 2}}$ \\
\hline 5211.477 & 19183.07 & 6 & & $30630^{o} o_{1 / 2}-49813_{2_{1 / 2}}$ \\
\hline 5214.087 & 19173.47 & 20 & 4 & $14169_{1_{1 / 2}}-33343_{1_{1 / 2}}^{o}$ \\
\hline 5220.239 & 19150.87 & 15 & 3 & $26917_{5_{1 / 2}}-46068^{o}{ }_{4_{1 / 2}}$ \\
\hline 5221.447 & 19146.44 & 10 & 1 & $21926_{2_{1 / 2}}-41073_{1_{1 / 2}}^{o}$ \\
\hline 5223.517 & 19138.86 & 2 & & $27517_{2_{1 / 2}}-46656^{o}{ }_{3_{1 / 2}}$ \\
\hline 5225.360 & 19132.11 & 10 & 1 & $25405_{1_{1 / 2}}-44537_{2_{1 / 2}}$ \\
\hline 5228.648 & 19120.08 & $40 \mathrm{cw}$ & $4 c w$ & $24147_{4_{1 / 2}}-43267^{0} 0_{1 / 2}$ \\
\hline 5228.756 & 19119.68 & 10 & & $23957_{3_{1 / 2}}-43077_{3_{1 / 2}}$ \\
\hline 5230.342 & 19113.88 & 12 & 1 & $22615_{3_{1 / 2}}-41729^{\circ} 3_{1 / 2}$ \\
\hline 5231.268 & 19110.50 & 15 & 1 & $11578_{1_{1 / 2}}-30689^{\circ} 0_{0_{1 / 2}}$ \\
\hline 5232.164 & 19107.23 & 5 & 1 & $20783_{3_{1 / 2}}-398910_{2_{1 / 2}}$ \\
\hline 5232.929 & 19104.43 & $1 h$ & & $30630^{o}{ }_{1_{1 / 2}}-49735_{1_{1 / 2}}$ \\
\hline 5236.130 & 19092.75 & 7 & 1 & $25174_{2_{1 / 2}}-44266^{o}{ }_{3_{1 / 2}}$ \\
\hline 5236.452 & 19091.58 & 5 & 1 & $27517_{2_{1 / 2}}^{1 / 2}-46608_{3_{1 / 2}}$ \\
\hline 5237.180 & 19088.93 & 2 & & $25598_{2_{1 / 2}}-44687_{3_{1 / 2}}$ \\
\hline 5237.521 & 19087.68 & 2 & & $22615_{3_{1 / 2}}-41703^{0}{ }_{4 / 2}$ \\
\hline 5238.247 & 19085.04 & 1 & & $24564_{5_{1 / 2}}-43649^{\circ}{ }_{4_{1 / 2}}$ \\
\hline
\end{tabular}


TABLE 3. Classified lines of Tc I

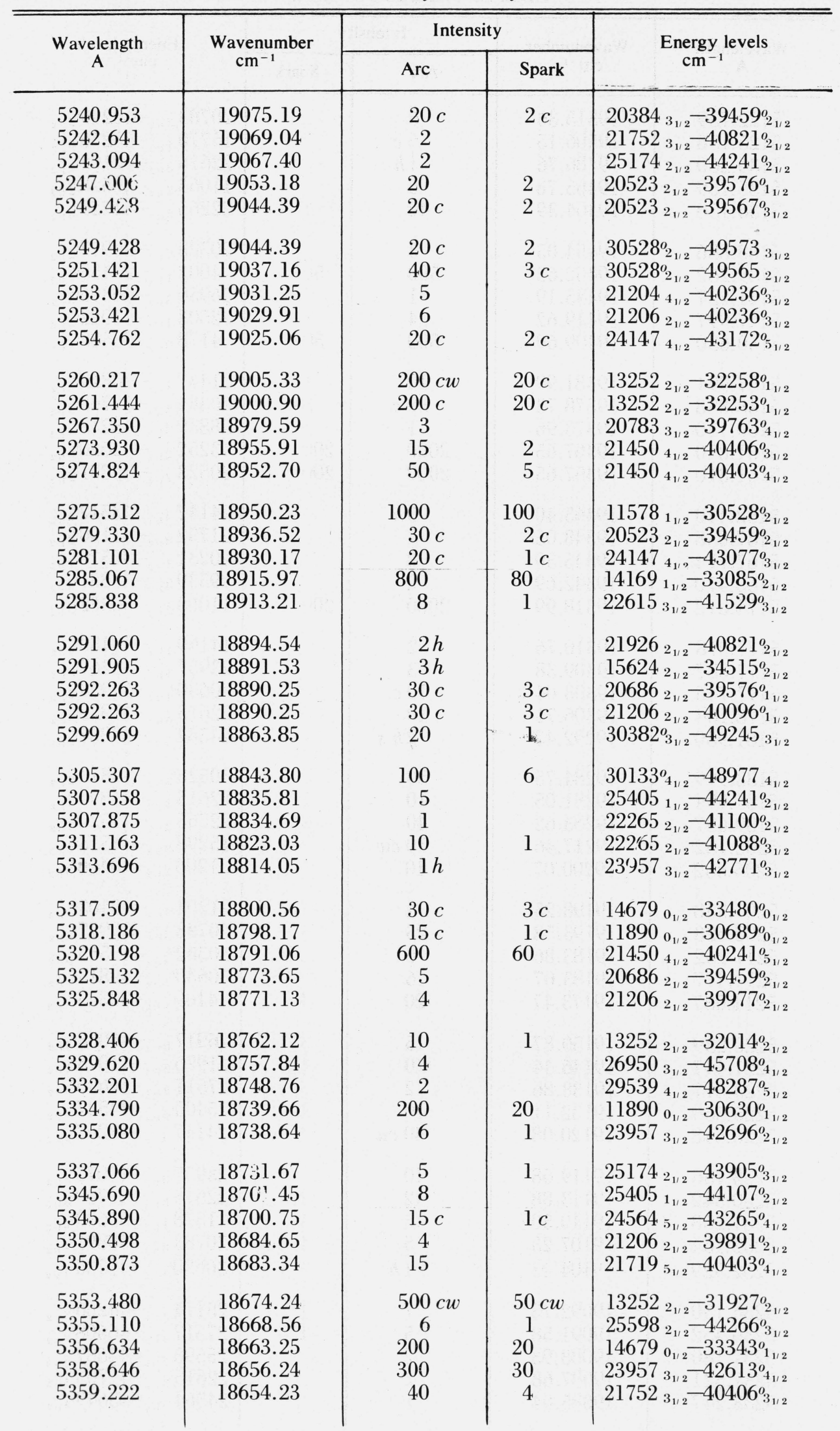


TABLE 3. Classified lines of $\mathrm{TC} \mathrm{I}$

\begin{tabular}{|c|c|c|c|c|}
\hline \multirow{2}{*}{$\underset{\text { W }}{\text { Wavelength }}$} & \multirow{2}{*}{$\begin{array}{l}\text { Wavenumber } \\
\mathrm{cm}^{-1}\end{array}$} & \multicolumn{2}{|c|}{ Intensity } & \multirow{2}{*}{$\begin{array}{c}\text { Energy levels } \\
\mathrm{cm}^{-1}\end{array}$} \\
\hline & & Arc & Spark & \\
\hline 5360.142 & 18651.03 & 200 & 20 & $21752_{3_{1^{\prime} 2}}-40403_{4_{1^{\prime} 2}}$ \\
\hline 5360.690 & 18649.12 & 15 & 1 & $21447_{1_{1 / 2}}-40096^{o}{ }_{1_{1 / 2}}$ \\
\hline 5362.420 & 18643.11 & $4 w d$ & & $21206_{2_{1 / 2}}-39849^{\circ}{ }_{3_{1 / 2}}$ \\
\hline 5364.050 & 18637.44 & 20 & 2 & $27971_{4_{1 / 2}}-46608_{3_{1 / 2}^{\circ}}^{0}$ \\
\hline 5366.082 & 18630.39 & 10 & $\overline{1}$ & $29233_{3_{1 / 2}}-47864^{0} o_{1 / 2}^{1 / 2}$ \\
\hline 5367.090 & 18626.89 & 2 & & $24147_{4_{1 / 2}}-42773_{5_{1^{\prime} / 2}}^{o}$ \\
\hline 5372.719 & 18607.37 & $1 h$ & & $24564_{5_{1 / 2}}-43172_{5_{1 / 2}}^{o}$ \\
\hline 5376.298 & 18594.98 & $10 h$ & $1 h$ & $30382^{o_{1 / 2}}-48977_{4_{1 / 2}}$ \\
\hline 5377.414 & 18591.13 & 20 & 2 & $25058_{3_{1 / 2}}-43649^{\circ}{ }_{4_{1 / 2}}$ \\
\hline 5378.457 & 18587.52 & 2 & 1 & $27517_{2_{1 / 2}}-46104_{2_{1 / 2}}^{o}$ \\
\hline 5378.970 & 18585.75 & $1 h$ & & $29233_{3_{1 / 2}}-47819^{o}{ }_{4_{1 / 2}}$ \\
\hline 5383.509 & 18570.08 & $30 c$ & $2 c$ & $22503_{1 / 2}-41073^{\circ}{ }_{1 / 2}$ \\
\hline 5385.832 & 18562.07 & $10 h$ & & $30382_{3_{1 / 2}}^{o}-48944_{2_{1 / 2}}$ \\
\hline 5386.872 & 18558.48 & 10 & 1 & $21204_{4_{1 / 2}}-39763_{4_{1 / 2}}^{o-1 / 2}$ \\
\hline 5387.630 & 18555.87 & $6 h$ & & $22265_{2_{1 / 2}}-40821_{2_{1 / 2}}^{0}$ \\
\hline 5390.214 & 18546.98 & 1 & & $21752_{3_{1 / 2}}-40299^{\circ} o_{2_{1 / 2}}$ \\
\hline 5394.569 & 18532.00 & $3 h$ & & $31406_{2_{1 / 2}}^{0}-49938_{2_{1 / 2}}^{1 / 2}$ \\
\hline 5395.149 & 18530.01 & 1 & & $21447_{1_{1 / 2}}-39977_{2_{1 / 2}}^{\circ}$ \\
\hline 5395.149 & 18530.01 & 1 & & $43971_{t_{1 / 2}}^{o_{1 / 2}}-62501_{3_{1 / 2}}^{-1 / 2}$ \\
\hline 5396.690 & 18524.72 & 2 & & $31414_{3_{1 / 2}}^{o}-49938_{2_{1 / 2}}$ \\
\hline 5397.539 & 18521.81 & 5 & & $21719_{5_{1 / 2}}-40241_{\tilde{5}_{1 / 2}}^{o}$ \\
\hline 5400.480 & 18511.72 & 20 & & $25598_{2_{1 / 2}}-44110^{o}{ }_{1_{1 / 2}}$ \\
\hline 5407.910 & 18486.29 & $50 c$ & $3 c$ & $21920_{2_{1 / 2}}^{-1 / 2}-40406_{3_{1 / 2}}^{0}$ \\
\hline 5411.928 & 18472.56 & 20 & 1 & $22615_{3_{1 / 2}}-41088_{3_{1 / 2}}$ \\
\hline 5413.624 & 18466.78 & $2 h$ & & $24147_{4_{1 / 2}}-42613_{4_{1 / 2}}^{o}$ \\
\hline 5417.121 & 18454.86 & 3 & & $28839_{5_{1 / 2}}-47294_{5_{1 / 2}}^{0}$ \\
\hline 5418.934 & 18448.68 & $2 h$ & & $19791_{4_{1 / 2}}-38240_{3_{1 / 2}}^{\circ}$ \\
\hline 5422.382 & 18436.95 & 2 & & $23460_{3_{1 / 2}}-41896_{4_{1 / 2}^{\circ}}^{\circ}$ \\
\hline 5423.048 & 18434.69 & $150 c$ & $15 c$ & $31503_{1_{1 / 2}}^{\circ}-49938_{2_{1 / 2}}$ \\
\hline 5425.956 & 18424.81 & 6 & 1 & $27971_{4_{1 / 2}}-46396_{3_{1 / 2}}^{0}$ \\
\hline 5430.712 & 18408.67 & 2 & & $25598_{2_{1 / 2}}-44007_{2_{1 / 2}}^{o}$ \\
\hline 5431.246 & 18406.86 & $15 h$ & 1 & $31406_{2_{1 / 2}}^{0}-49813_{2_{1 / 2}}$ \\
\hline 5432.437 & 18402.83 & 5 & & $30382_{3_{1 / 2}}^{o^{1 / 2}}-48784_{3_{1 / 2}}^{1 / 2}$ \\
\hline 5433.894 & 18397.89 & 1 & & $24147_{4_{1 / 2}}-42545_{4_{1 / 2}^{\circ}}^{\circ}$ \\
\hline 5438.774 & 18381.38 & $2 h$ & & $20384_{3_{1 / 2}}-38766_{3_{1 / 2}}^{0}$ \\
\hline 5441.440 & 18372.38 & 2 & & $21926_{2_{1 / 2}}-40299^{o}{ }_{2_{1 / 2}}$ \\
\hline 5441.440 & 18372.38 & 2 & & $251744^{1 / 2}-43546^{\circ}$ \\
\hline 5442.071 & 18370.25 & $4 c w$ & & $21206_{2_{1 / 2}}-39576_{1_{1 / 2}}^{\circ}$ \\
\hline 5444.258 & 18362.87 & 50 & 5 & $21204_{4_{1 / 2}}^{-1 / 2}-39567_{3_{1 / 2}}$ \\
\hline 5447.402 & 18352.27 & 200 & 20 & $13252_{2_{1 / 2}}-31605_{3_{1 / 2}}^{o}$ \\
\hline 5447.800 & 18350.93 & $20 c$ & & $30067^{0} \tilde{5}_{1 / 2}-48418_{4_{1 / 2}}$ \\
\hline 5451.900 & 18337.13 & $500 c$ & $50 c$ & $19903_{3_{1 / 2}}^{1 / 2}-38240_{3_{1 / 2}}^{\circ}$ \\
\hline 5454.124 & 18329.65 & 3 & & $30133^{\circ}{ }_{4_{1 / 2}}-48462_{3_{1 / 2}}$ \\
\hline 5455.112 & 18326.33 & $20 w d$ & & $22503_{1_{1 / 2}}-40829^{\circ} 0_{1 / 2}^{1 / 2}$ \\
\hline 5458.159 & 18316.10 & $30 \mathrm{cw}$ & & $21920_{2_{1 / 2}}^{1 / 2}-40236_{3_{1 / 2}}^{0}$ \\
\hline 5459.118 & 18312.88 & $2 c$ & & $21450_{4_{1 / 2}}-39763_{4_{1^{\prime}}}$ \\
\hline 5460.747 & 18307.42 & 10 & & $25598_{2_{1 / 2}}-43905^{0} o_{3_{1 / 2}}$ \\
\hline 5467.471 & 18284.91 & 10 & & $30133_{4_{1 / 2}}^{-1 / 2}-48418_{4_{1 / 2}}$ \\
\hline 5468.768 & 18280.57 & 3 & & $29539_{4_{1 / 2}}-47819^{\circ}{ }_{4_{1 / 2}}$ \\
\hline 5471.962 & 18269.90 & 300 & & $23460_{3_{1 / 2}}-41729^{\circ}{ }_{3_{1 / 2}}$ \\
\hline
\end{tabular}


TABLE 3. Classified lines of TC I

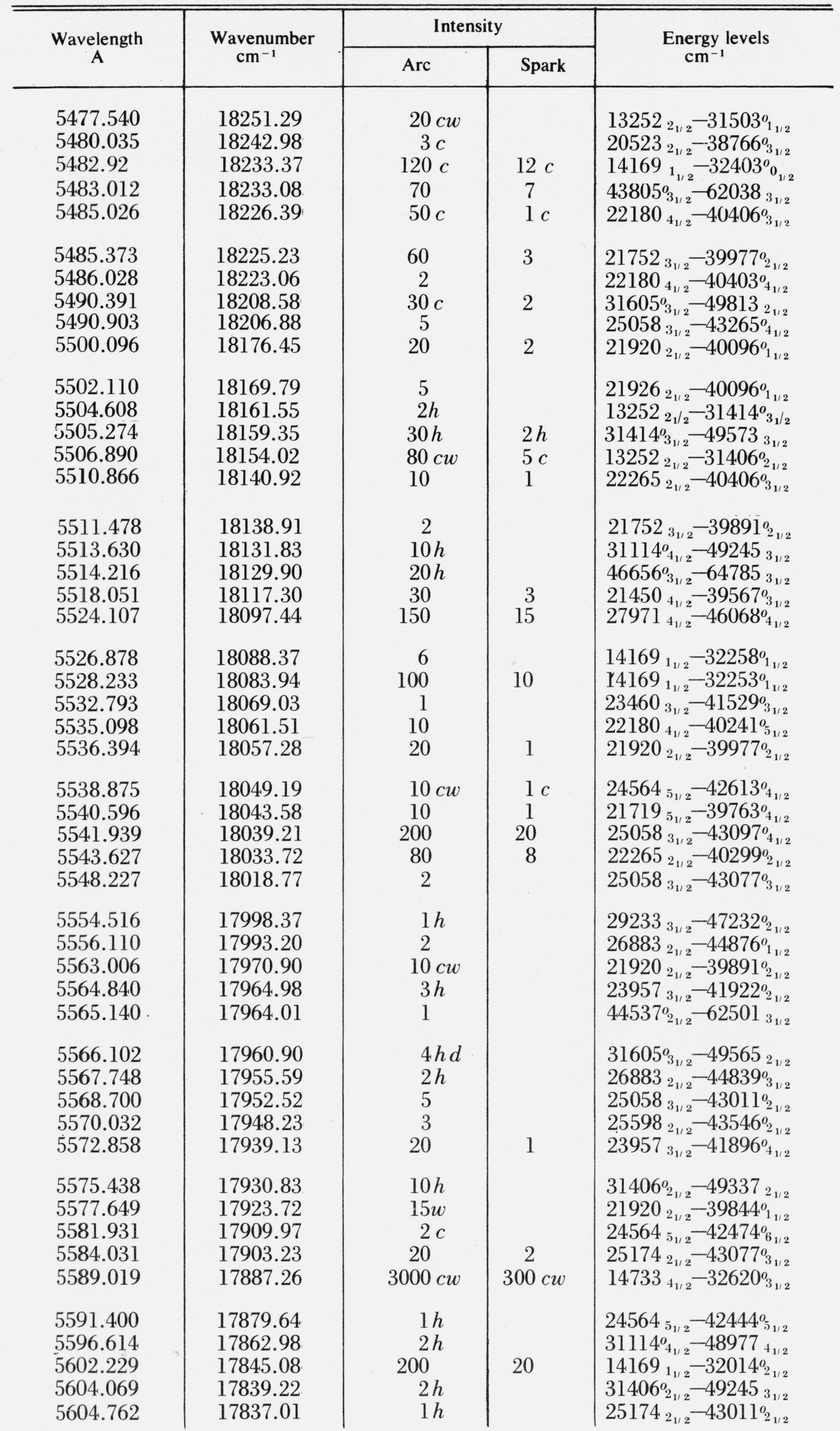


TABLE 3. Classified lines of $\mathrm{TC} \mathrm{I}$

\begin{tabular}{|c|c|c|c|c|}
\hline \multirow{2}{*}{$\begin{array}{c}\text { Wavelength } \\
\text { A }\end{array}$} & \multirow{2}{*}{$\begin{array}{l}\text { Wavenumber } \\
\mathrm{cm}^{-1}\end{array}$} & \multicolumn{2}{|c|}{ Intensity } & \multirow{2}{*}{$\begin{array}{c}\text { Energy levels } \\
\mathrm{cm}^{-1}\end{array}$} \\
\hline & & Arc & Spark & \\
\hline $\begin{array}{l}5606.376 \\
5606.376 \\
5611.482 \\
5613.863 \\
5615.272\end{array}$ & $\begin{array}{l}17831.88 \\
17831.88 \\
17815.65 \\
17808.10 \\
17803.63\end{array}$ & $\begin{array}{l}10 h \\
10 h \\
20 \\
2 h \\
8\end{array}$ & $\begin{array}{l}2 \\
1\end{array}$ & 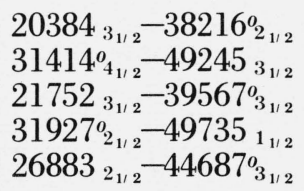 \\
\hline $\begin{array}{l}5615.272 \\
5616.795 \\
5617.651 \\
5620.450 \\
5625.232\end{array}$ & $\begin{array}{l}17803.63 \\
17798.80 \\
17796.09 \\
17787.23 \\
17772.11\end{array}$ & $\begin{array}{r}8 \\
10 \\
7 \\
2000 c \\
6\end{array}$ & $\begin{array}{r}1 \\
1 \\
200\end{array}$ & $\begin{array}{l}30060_{2_{1 / 2}}-47864^{o} 3_{1 / 2} \\
32014^{o} o_{2_{1 / 2}}-49813_{2_{1 / 2}} \\
20523_{2_{1 / 2}}-38319^{o} o_{1_{1 / 2}} \\
15298_{3_{1 / 2}}-33085^{o}{ }_{2_{1 / 2}} \\
23957_{3_{1 / 2}}-41729^{o}{ }_{3_{1 / 2}}\end{array}$ \\
\hline $\begin{array}{l}5628.956 \\
5629.568 \\
5629.937 \\
5636.253 \\
5637.792\end{array}$ & $\begin{array}{l}17760.35 \\
17758.42 \\
17757.25 \\
17737.36 \\
17732.51\end{array}$ & $\begin{array}{c}1 \\
5 \\
300 \\
30 \\
8 h\end{array}$ & $\begin{array}{r}30 \\
3 \\
1\end{array}$ & $\begin{array}{l}44839^{o}{ }_{3_{1 / 2}}-62599_{4_{1 / 2}} \\
25058_{3_{1 / 2}}-42817^{o}{ }_{4_{1 / 2}} \\
14169_{1_{1 / 2}}-31927^{\circ} o_{2_{1 / 2}} \\
27971_{4_{1 / 2}}-45708^{o}{ }_{4_{1 / 2}} \\
31605^{\circ}{ }_{3_{1 / 2}}-49337_{2_{1 / 2}}\end{array}$ \\
\hline $\begin{array}{l}5640.782 \\
5642.132 \\
5643.940 \\
5644.357 \\
5644.944\end{array}$ & $\begin{array}{l}17723.11 \\
17718.87 \\
17713.20 \\
17711.89 \\
17710.05\end{array}$ & $\begin{array}{r}40 c \\
1500 \\
6 \\
12 \\
800\end{array}$ & $\begin{array}{r}2 c \\
150 \\
1 \\
80\end{array}$ & $\begin{array}{l}14679_{0_{1 / 2}}-32403^{o} 0_{1 / 2} \\
15624_{2_{1 / 2}}-33343^{o} 1_{1 / 2} \\
25058_{3_{1 / 2}}-42771^{0} o_{1 / 2} \\
22265_{2_{1 / 2}}-39977^{\circ} o_{2_{1 / 2}} \\
15770_{1_{1 / 2}}-33480^{\circ} 0_{0_{1 / 2}}\end{array}$ \\
\hline $\begin{array}{l}5645.694 \\
5650.259 \\
5652.909 \\
5654.338 \\
5657.490\end{array}$ & $\begin{array}{l}17707.70 \\
17693.39 \\
17685.09 \\
17680.62 \\
17670.77\end{array}$ & $\begin{array}{l}2 \\
1 \\
1 \\
6 \\
3\end{array}$ & & $\begin{array}{l}21752_{3_{1 / 2}}-39459^{0} o_{2_{1 / 2}} \\
20523_{2_{1 / 2}}-38216^{o} o_{2_{1 / 2}} \\
32253^{o} o_{1_{1 / 2}}-49938_{2_{1 / 2}} \\
32258^{o}{ }_{1_{1 / 2}}-49938_{2_{1 / 2}} \\
31114^{o}{ }_{4_{1 / 2}}-487844_{3_{1 / 2}}\end{array}$ \\
\hline $\begin{array}{l}5658.106 \\
5661.557 \\
5662.104 \\
5664.232 \\
5664.900\end{array}$ & $\begin{array}{l}17668.85 \\
17658.08 \\
17656.37 \\
17649.74 \\
17647.66\end{array}$ & $\begin{array}{r}8 \\
20 \\
10 \\
4 \\
40 \mathrm{cw}\end{array}$ & $\begin{array}{l}1 \\
2 \\
1 \\
2 c w\end{array}$ & $\begin{array}{l}25598_{2_{1 / 2}}-43267^{0}{ }_{3_{1 / 2}} \\
26917_{5_{1 / 2}}-44576^{o}{ }_{4_{1 / 2}} \\
21920_{2_{1 / 2}}-39576^{o}{ }_{1_{1 / 2}} \\
21926_{2_{1 / 2}}-39576^{o}{ }_{1_{1 / 2}} \\
21920_{2_{1 / 2}}-39567^{0} o_{3_{1 / 2}}\end{array}$ \\
\hline $\begin{array}{l}5667.058 \\
5667.058 \\
5672.153 \\
5675.178 \\
5678.478\end{array}$ & $\begin{array}{l}17640.94 \\
17640.94 \\
17625.09 \\
17615.70 \\
17605.46\end{array}$ & $\begin{array}{l}15 \\
15 \\
60 \\
5 h s \\
50\end{array}$ & $\begin{array}{l}6 \\
2\end{array}$ & 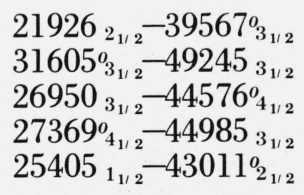 \\
\hline $\begin{array}{l}5680.973 \\
5682.298 \\
5687.301 \\
5688.730 \\
5689.046\end{array}$ & $\begin{array}{l}17597.73 \\
17593.63 \\
17578.15 \\
17573.74 \\
17572.76\end{array}$ & $\begin{array}{l}15 \\
1 h \\
200 \\
20 \\
200\end{array}$ & $\begin{array}{r}20 \\
2 \\
20\end{array}$ & 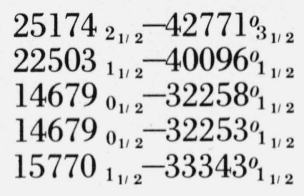 \\
\hline $\begin{array}{l}5692.212 \\
5694.330 \\
5696.056 \\
5696.626 \\
5702.894\end{array}$ & $\begin{array}{l}17562.99 \\
17556.45 \\
17551.13 \\
17549.38 \\
17530.09\end{array}$ & $\begin{array}{l}15 h \\
15 c \\
20 h \\
18 c w \\
5 h\end{array}$ & $\begin{array}{l}1 \\
2 c \\
2 \\
2 c\end{array}$ & $\begin{array}{l}31414^{o}{ }_{4_{1 / 2}}-48977_{{ }_{1 / 2}} \\
24147^{4_{1 / 2}}-41703^{o}{ }_{4_{1 / 2}} \\
32014^{0} o_{2_{1 / 2}}-49565_{2_{1 / 2}} \\
27369^{\circ}{ }_{4_{1 / 2}}-44919_{4_{1 / 2}} \\
31414^{o_{3 / 2}}-48944_{2_{1 / 2}}\end{array}$ \\
\hline $\begin{array}{l}5705.403 \\
5718.775 \\
5719.542 \\
5720.205 \\
5721.090\end{array}$ & $\begin{array}{l}17522.38 \\
17481.41 \\
17479.06 \\
17477.04 \\
17474.339\end{array}$ & $\begin{array}{l}1 h \\
1 h \\
10 \\
1 h \\
10 h\end{array}$ & $\begin{array}{l}1 \\
1 h\end{array}$ & 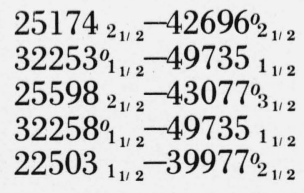 \\
\hline
\end{tabular}


TABLE 3. Classified lines of $\mathrm{Tc} \mathrm{I}$

\begin{tabular}{|c|c|c|c|c|}
\hline \multirow{2}{*}{$\begin{array}{c}\text { Wavelength } \\
\text { A }\end{array}$} & \multirow{2}{*}{$\begin{array}{l}\text { Wavenumber } \\
\mathrm{cm}^{-1}\end{array}$} & \multicolumn{2}{|c|}{ Intensity } & \multirow{2}{*}{$\underset{\mathrm{cm}^{-1}}{\text { Energy levels }}$} \\
\hline & & Arc & Spark & \\
\hline $\begin{array}{l}5723.255 \\
5725.314 \\
5732.791 \\
5734.691 \\
5734.691\end{array}$ & $\begin{array}{l}17467.72 \\
17461.44 \\
17438.67 \\
17432.89 \\
17432.89\end{array}$ & $\begin{array}{l}2 h \\
700 \\
3 \\
2 h \\
2 h\end{array}$ & 70 & 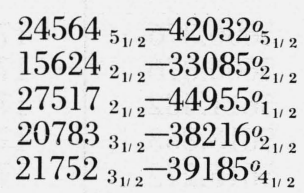 \\
\hline $\begin{array}{l}5735.636 \\
5737.543 \\
5739.479 \\
5741.301 \\
5741.943\end{array}$ & $\begin{array}{l}17430.02 \\
17424.23 \\
17418.35 \\
17412.82 \\
17410.87\end{array}$ & $\begin{array}{l}3 \\
5 w \\
1 h \\
5 \\
10 h w\end{array}$ & $1 h$ & $\begin{array}{l}26883_{2_{1 / 2}}-44313^{o}{ }_{1_{1 / 2}} \\
10516_{3_{1 / 2}}-27940^{o} o_{2_{1 / 2}} \\
41896^{o}{ }_{4_{1 / 2}}-59315_{5_{1 / 2}} \\
25598_{2_{1 / 2}}-43011_{2_{1 / 2}}^{o} \\
27660^{o} o_{3_{1 / 2}}-45071_{2_{1 / 2}}\end{array}$ \\
\hline $\begin{array}{l}5748.307 \\
5749.542 \\
5751.050 \\
5754.775 \\
5755.199\end{array}$ & $\begin{array}{l}17391.60 \\
17387.86 \\
17383.30 \\
17372.05 \\
17370.77\end{array}$ & $\begin{array}{r}3 \\
10 c \\
1 h \\
20 h \\
3 h\end{array}$ & & $\begin{array}{l}30060_{2_{1 / 2}}-47452_{2_{1 / 2}}^{o} \\
22503_{1_{1 / 2}}-39891_{2_{1 / 2}}^{o} \\
26883_{2_{1 / 2}}-44266^{o} \\
31605_{3_{1 / 2}}^{o} 3_{3_{1 / 2}}-48977_{4_{1 / 2}} \\
31414_{3_{1 / 2}}^{o}-48784_{3_{1 / 2}}^{o_{12}}\end{array}$ \\
\hline $\begin{array}{l}5758.298 \\
5760.389 \\
5761.734 \\
5762.449 \\
5765.698\end{array}$ & $\begin{array}{l}17361.42 \\
17355.12 \\
17351.07 \\
17348.92 \\
17339.14\end{array}$ & $\begin{array}{l}1 h \\
2 \\
1 \\
5 \\
5 h\end{array}$ & & 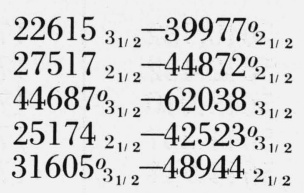 \\
\hline $\begin{array}{l}5767.232 \\
5767.546 \\
5768.002 \\
5769.704 \\
5770.249\end{array}$ & $\begin{array}{l}17334.53 \\
17333.58 \\
17332.21 \\
17327.10 \\
17325.47\end{array}$ & $\begin{array}{l}4 h c \\
6 \\
2 \\
3 \\
4\end{array}$ & & $\begin{array}{l}14169^{1_{1 / 2}}-31503^{o}{ }_{1_{1 / 2}} \\
47452^{o} o_{2_{1 / 2}}-64785_{3_{1 / 2}} \\
24564_{5_{1 / 2}}-41896^{\circ}{ }_{4_{1 / 2}} \\
27940^{\circ}{ }_{2_{1 / 2}}-45267_{1_{1 / 2}} \\
27660^{o}{ }_{3_{1 / 2}}-44985_{3_{1 / 2}}\end{array}$ \\
\hline $\begin{array}{l}5771.468 \\
5772.500 \\
5773.656 \\
5775.069 \\
5777.360\end{array}$ & $\begin{array}{l}17321.81 \\
17318.71 \\
17315.24 \\
17311.01 \\
17304.14\end{array}$ & $\begin{array}{c}500 c \\
1 h \\
20 \\
10 c w \\
1\end{array}$ & $\begin{array}{l}50 c \\
2 \\
1 c\end{array}$ & $\begin{array}{l}15298_{3_{1 / 2}}-32620^{o} 3_{1 / 2} \\
26788_{2_{1 / 2}}-44107^{\circ}{ }_{2_{1 / 2}} \\
15770_{1_{1 / 2}}-33085^{\circ} o_{2_{1 / 2}} \\
22265_{2_{1 / 2}}-39576^{o}{ }_{1_{1 / 2}} \\
31114_{4_{1 / 2}}^{o}-48418_{4_{1 / 2}}\end{array}$ \\
\hline $\begin{array}{l}5777.976 \\
5792.474 \\
5799.850 \\
5802.433 \\
5805.214\end{array}$ & $\begin{array}{l}17302.30 \\
17258.99 \\
17237.04 \\
17229.37 \\
17221.11\end{array}$ & $\begin{array}{l}6 \\
10 c w \\
80 c \\
10 \\
20\end{array}$ & $\begin{array}{l}1 \\
8 c \\
1 \\
2\end{array}$ & $\begin{array}{l}22265_{2_{1 / 2}}-39567^{0} 3_{3_{1 / 2}} \\
27660^{o}{ }_{3_{1 / 2}}-44919_{4_{1 / 2}} \\
14169_{1_{1 / 2}}-31406^{o}{ }_{2_{1 / 2}} \\
28839_{5_{1 / 2}}-46068^{o}{ }_{4_{1 / 2}} \\
30060_{2_{1 / 2}}-47281_{2_{1 / 2}}^{o}\end{array}$ \\
\hline $\begin{array}{l}5805.525 \\
5806.126 \\
5814.241 \\
5814.639 \\
5815.038\end{array}$ & $\begin{array}{l}17220.19 \\
17218.41 \\
17194.38 \\
17193.20 \\
17192.02\end{array}$ & $\begin{array}{c}10 \\
2 h \\
100 c w \\
2 \\
1 h\end{array}$ & $\begin{array}{c}1 \\
10 c\end{array}$ & $\begin{array}{l}26917_{5_{1 / 2}}-44138^{o}{ }_{5_{1 / 2}} \\
26788_{2_{1 / 2}}-44007^{\circ}{ }_{2_{1 / 2}} \\
22265_{2_{1 / 2}}-39459^{\circ}{ }_{2_{1 / 2}} \\
32620^{\circ}{ }_{3_{1 / 2}}-49813_{2_{1 / 2}} \\
250588_{3_{1 / 2}}-42250^{\circ}{ }_{2_{1 / 2}}\end{array}$ \\
\hline $\begin{array}{l}5819.176 \\
5821.310 \\
5823.196 \\
5827.189 \\
5831.477\end{array}$ & $\begin{array}{l}17179.80 \\
17173.50 \\
17167.94 \\
17156.17 \\
17143.56\end{array}$ & $\begin{array}{l}15 h l \\
10 \\
8 \\
10 \\
200\end{array}$ & $\begin{array}{r}1 \\
1 \\
1 \\
20\end{array}$ & $\begin{array}{l}31605^{o}{ }_{3_{1 / 2}}-48784_{3_{1 / 2}} \\
25598_{2_{1 / 2}}-42771^{o} 3_{1 / 2} \\
27940^{o}{ }_{2_{1 / 2}}-45108_{1_{1 / 2}} \\
26950_{3_{1 / 2}}-44107^{\circ} o_{2_{1 / 2}} \\
10516_{3_{1 / 2}}-27660^{o}{ }_{3_{1 / 2}}\end{array}$ \\
\hline $\begin{array}{l}5831.858 \\
5832.836 \\
5835.865 \\
5836.329 \\
5838.366\end{array}$ & $\begin{array}{l}17142.44 \\
17139.56 \\
17130.67 \\
17129.30 \\
17123.33\end{array}$ & $\begin{array}{r}2 \\
2 \\
6 \\
150 c \\
10\end{array}$ & $\begin{array}{c}1 \\
15 c \\
1\end{array}$ & $\begin{array}{l}23957_{3_{1 / 2}}-41100^{o}{ }_{2_{1 / 2}} \\
26950_{3_{1 / 2}}-44090^{o}{ }_{3_{1 / 2}} \\
23957_{3_{1 / 2}}-41088^{\circ} o_{3_{1 / 2}} \\
13252_{2_{1 / 2}}-30382^{o} o_{3_{1 / 2}} \\
26883_{2_{1 / 2}}-44007^{\circ}{ }_{2_{1 / 2}}\end{array}$ \\
\hline
\end{tabular}


TABLE 3. Classified lines of TC I

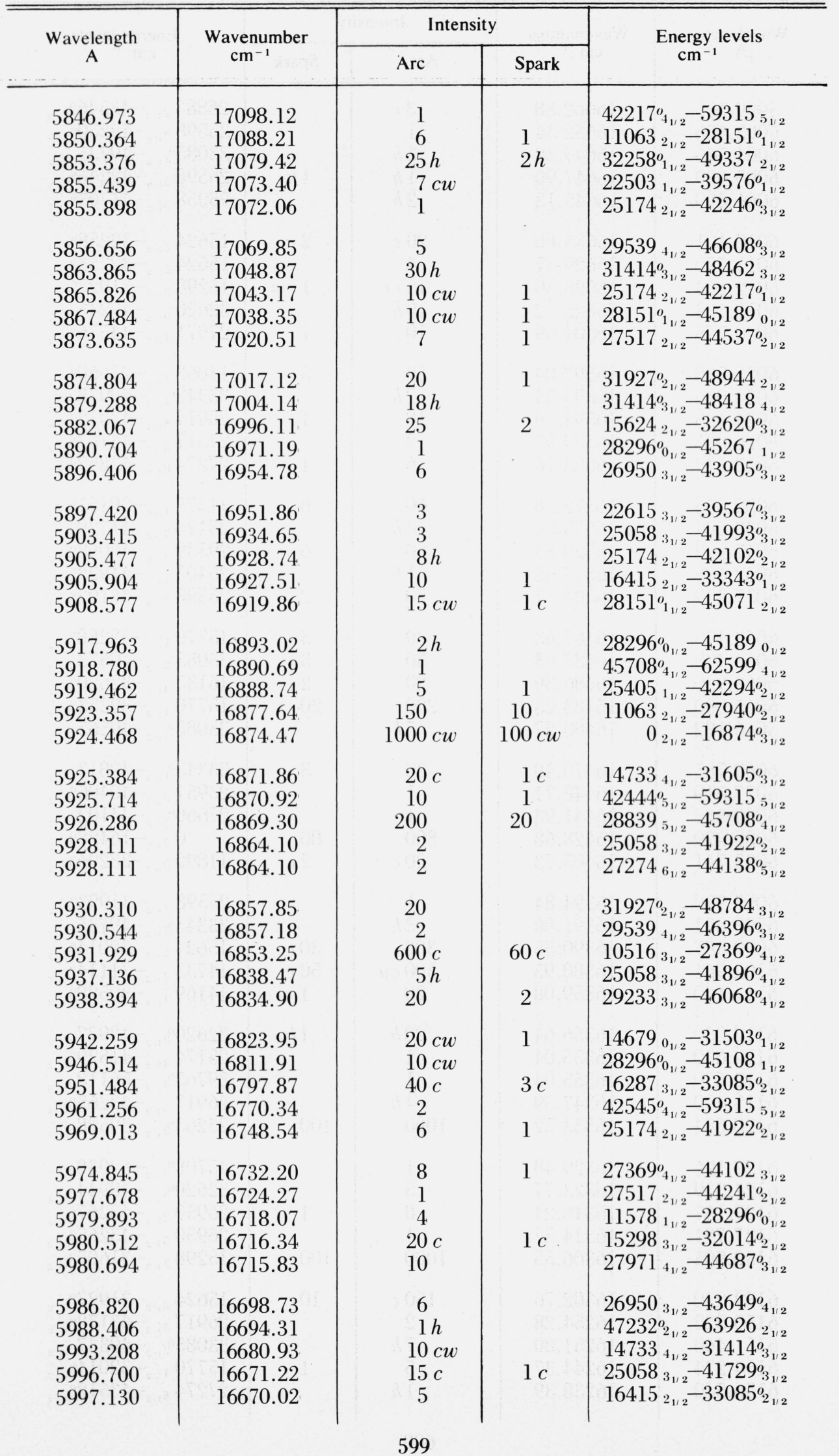


TABLE 3. Classified lines of $\mathrm{TC} \mathrm{I}$

\begin{tabular}{|c|c|c|c|c|}
\hline \multirow{2}{*}{$\begin{array}{c}\text { Wavelength } \\
\text { A }\end{array}$} & \multirow{2}{*}{$\begin{array}{l}\text { Wavenumber } \\
\mathrm{cm}^{-1}\end{array}$} & \multicolumn{2}{|c|}{ Intensity } & \multirow{2}{*}{$\underset{\mathrm{cm}^{-1}}{\text { Energy levels }}$} \\
\hline & & Arc & Spark & \\
\hline $\begin{array}{l}5999.701 \\
6003.496 \\
6004.566 \\
6005.099 \\
6006.099\end{array}$ & $\begin{array}{l}16662.88 \\
16652.34 \\
16649.38 \\
16647.90 \\
16645.13\end{array}$ & $\begin{array}{l}3 c \\
1 \\
3 h \\
1 h \\
2 h\end{array}$ & $1 h$ & $\begin{array}{l}26883_{2_{1 / 2}}-43546^{o}{ }_{2_{1 / 2}} \\
255988_{2_{1 / 2}}-42250^{o} o_{2_{1 / 2}} \\
33085^{o} o_{2_{1 / 2}}-49735_{1_{1 / 2}} \\
25598_{2_{1 / 2}}-42246^{o}{ }_{3_{1 / 2}} \\
25058_{3_{1 / 2}}-41703^{o}{ }_{4_{1 / 2}}\end{array}$ \\
\hline $\begin{array}{l}6010.191 \\
6011.790 \\
6012.104 \\
6013.183 \\
6020.726\end{array}$ & $\begin{array}{l}16633.80 \\
16629.37 \\
16628.50 \\
16625.52 \\
16604.69\end{array}$ & $\begin{array}{l}20 c \\
4 \\
15 c w \\
10 h \\
10\end{array}$ & $\begin{array}{l}2 c \\
1 c w \\
1\end{array}$ & $\begin{array}{l}156244_{2_{1 / 2}}-32258^{o}{ }_{1_{1 / 2}} \\
156244_{2_{1 / 2}}-32253^{o}{ }_{1_{1 / 2}} \\
152988_{3_{1 / 2}}-31927^{\circ} o_{2_{1 / 2}} \\
32620^{o} o_{3_{1 / 2}}-49245_{3_{1 / 2}} \\
27971_{4_{1 / 2}}-44576^{o}{ }_{4_{1 / 2}}\end{array}$ \\
\hline $\begin{array}{l}6023.500 \\
6024.046 \\
6024.423 \\
6026.085 \\
6028.542\end{array}$ & $\begin{array}{l}16597.04 \\
16595.54 \\
16594.50 \\
16589.92 \\
16583.16\end{array}$ & $\begin{array}{l}50 \\
2 h \\
10 \\
2 \\
6\end{array}$ & $\begin{array}{l}5 \\
1 \\
1\end{array}$ & 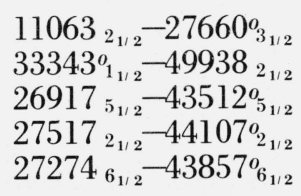 \\
\hline $\begin{array}{l}6032.355 \\
6038.504 \\
6047.992 \\
6052.685 \\
6057.249\end{array}$ & $\begin{array}{l}16572.68 \\
16555.80 \\
16529.83 \\
16517.02 \\
16504.57\end{array}$ & $\begin{array}{l}60 \\
4 h \\
60 \\
1 h \\
20\end{array}$ & $\begin{array}{l}6 \\
6 \\
2\end{array}$ & $\begin{array}{l}115788_{1_{1 / 2}}-28151^{o}{ }_{1_{1 / 2}} \\
251744_{2_{1 / 2}}-417290^{o}{ }_{3_{1 / 2}} \\
295399_{4_{1 / 2}}-46068^{o}{ }_{4_{1 / 2}} \\
254055_{1_{1 / 2}}-41922^{o} o_{{ }_{1 / 2}} \\
25598_{2_{1 / 2}}-42102^{o} o_{2_{1 / 2}}\end{array}$ \\
\hline $\begin{array}{l}6063.473 \\
6063.473 \\
6063.930 \\
6065.090 \\
6066.182\end{array}$ & $\begin{array}{l}16487.63 \\
16487.63 \\
16486.39 \\
16483.23 \\
16480.27\end{array}$ & $\begin{array}{c}30 \\
30 \\
20 c \\
200 \\
5 h\end{array}$ & $\begin{array}{r}3 \\
3 \\
2 \\
20\end{array}$ & $\begin{array}{l}15770_{1_{1 / 2}}-32258^{o}{ }_{1_{1 / 2}} \\
33085_{2_{1 / 2}}^{o}-49573_{3_{1 / 2}}^{o_{1 / 2}}-32620^{o} 3_{1 / 2} \\
16133_{4_{1 / 2}}-32253_{1_{1 / 2}}^{o} \\
15770_{1_{1 / 2}}-322565_{2_{1 / 2}}^{o}\end{array}$ \\
\hline $\begin{array}{l}6069.785 \\
6077.819 \\
6080.327 \\
6085.229 \\
6093.741\end{array}$ & $\begin{array}{l}16470.48 \\
16448.71 \\
16441.93 \\
16428.68 \\
16405.73\end{array}$ & $\begin{array}{r}30 \\
1 \\
1 \\
800 \\
20 c\end{array}$ & $\begin{array}{l}80 \\
2 c\end{array}$ & 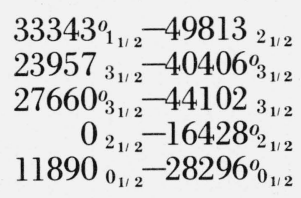 \\
\hline $\begin{array}{l}6097.790 \\
6098.891 \\
6099.387 \\
6102.961 \\
6111.120\end{array}$ & $\begin{array}{l}16394.84 \\
16391.88 \\
16390.55 \\
16380.95 \\
16359.08\end{array}$ & $\begin{array}{l}1 \\
3 h \\
300 \\
500 \mathrm{cw} \\
20\end{array}$ & $\begin{array}{c}30 \\
50 c \\
1\end{array}$ & $\begin{array}{l}25598_{2_{1 / 2}}-41993_{3_{1 / 2}}^{o} \\
33343_{1_{1 / 2}}^{o}-497355_{1_{1 / 2}} \\
15624{ }_{2_{1 / 2}}-32014^{o} o_{1 / 2} \\
14733_{4_{1 / 2}}-311144_{4_{1 / 2}}^{o} \\
14169_{1_{1 / 2}}-30528_{2_{1 / 2}}^{o}\end{array}$ \\
\hline $\begin{array}{l}6112.030 \\
6112.630 \\
6112.630 \\
6115.490 \\
6120.684\end{array}$ & $\begin{array}{l}16356.64 \\
16355.04 \\
16355.04 \\
16347.39 \\
16333.52\end{array}$ & $\begin{array}{c}20 h \\
1 \\
1 \\
4 h \\
1000\end{array}$ & 100 & $\begin{array}{l}32620_{3_{1 / 2}}^{o}-48977_{4_{1 / 2}} \\
25174_{2_{1 / 2}}-41529^{o}{ }_{3_{1 / 2}} \\
39763^{o}{ }_{4_{1 / 2}}-56118_{5_{1 / 2}} \\
26917_{5_{1 / 2}}-43265^{o}{ }_{4_{1 / 2}} \\
23265_{3_{1 / 2}}^{o}-39598_{2_{1 / 2}}^{o}\end{array}$ \\
\hline $\begin{array}{l}6122.196 \\
6124.339 \\
6127.167 \\
6127.791 \\
6130.805\end{array}$ & $\begin{array}{l}16329.48 \\
16323.77 \\
16316.24 \\
16314.57 \\
16306.55\end{array}$ & $\begin{array}{r}1 \\
5 \\
8 \\
1 \\
1000\end{array}$ & $\begin{array}{r}1 \\
100\end{array}$ & 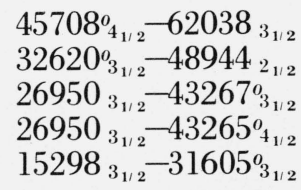 \\
\hline $\begin{array}{l}6132.230 \\
6150.522 \\
6151.460 \\
6154.274 \\
6156.540\end{array}$ & $\begin{array}{l}16302.76 \\
16254.28 \\
16251.80 \\
16244.37 \\
16238.39\end{array}$ & $\begin{array}{c}150 c \\
2 \\
7 h \\
5 \\
1 h\end{array}$ & $10 c$ & 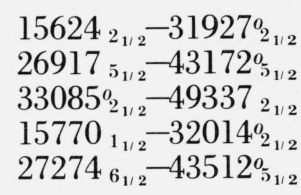 \\
\hline
\end{tabular}


TABLE 3. Classified lines of $\mathrm{TC} \mathrm{I}$

\begin{tabular}{|c|c|c|c|c|}
\hline \multirow{2}{*}{$\begin{array}{c}\text { Wavelength } \\
\text { A }\end{array}$} & \multirow{2}{*}{$\begin{array}{l}\text { Wavenumber } \\
\mathrm{cm}^{-1}\end{array}$} & \multicolumn{2}{|c|}{ Intensity } & \multirow{2}{*}{$\begin{array}{l}\text { Energy levels } \\
\mathrm{cm}^{-1}\end{array}$} \\
\hline & & Arc & Spark & \\
\hline $\begin{array}{l}6163.603 \\
6174.723 \\
6182.668 \\
6183.771 \\
6184.698\end{array}$ & $\begin{array}{l}16219.788 \\
16190.578 \\
16169.773 \\
16166.889 \\
16164.465\end{array}$ & $\begin{array}{l}8 \\
1 h \\
20 \\
10 \\
100\end{array}$ & $\begin{array}{l}1 \\
2 \\
1 \\
2\end{array}$ & $\begin{array}{l}254055_{1_{1 / 2}}-41625^{o}{ }_{1_{1 / 2}} \\
25598_{2_{1 / 2}}-41788^{o} o_{1 / 2} \\
29539_{4_{1 / 2}}-45708^{o} o_{d_{1 / 2}} \\
27971_{4_{1 / 2}}-44138_{\tilde{J}_{1 / 2}}^{o} \\
32620_{3_{1 / 2}}^{o}-48784_{3_{1 / 2}}^{o}\end{array}$ \\
\hline $\begin{array}{l}6185.908 \\
6187.702 \\
6192.665 \\
6195.384 \\
6197.312\end{array}$ & $\begin{array}{l}16161.304 \\
16156.618 \\
16143.670 \\
16136.585 \\
16131.565\end{array}$ & $\begin{array}{r}5 \\
3 \\
800 \\
40 \\
1\end{array}$ & $\begin{array}{r}80 \\
2\end{array}$ & $\begin{array}{l}27940^{o}{ }_{2_{1 / 2}}-44102_{3_{1 / 2}} \\
15770_{1_{1 / 2}}-31927^{o} o_{2_{1 / 2}} \\
23455^{0} o_{2_{1 / 2}}-39598_{2_{1 / 2}} \\
27660^{o} o_{3_{1 / 2}}-43796_{2_{1 / 2}} \\
25598_{2_{1 / 2}}-41729^{o} o_{3_{1 / 2}}\end{array}$ \\
\hline $\begin{array}{l}6198.853 \\
6202.630 \\
6202.902 \\
6204.446 \\
6214.032\end{array}$ & $\begin{array}{l}16127.554 \\
16117.734 \\
16117.027 \\
16113.016 \\
16088.160\end{array}$ & $\begin{array}{l}4 h \\
5 \\
3 \\
4 \\
30\end{array}$ & 2 & $\begin{array}{l}268832_{2_{1 / 2}}-43011_{2_{1 / 2}}^{o} \\
431970_{6_{1 / 2}}-59315_{5_{1 / 2}}^{o_{2}} \\
26788_{2_{1 / 2}}-42905_{2_{1 / 2}}^{o} \\
26788_{2_{1 / 2}}-42901_{1_{1 / 2}}^{o} \\
27940^{o} ._{2_{1 / 2}}-44028_{1_{1 / 2}}\end{array}$ \\
\hline $\begin{array}{l}6218.473 \\
6232.076 \\
6236.621 \\
6236.865 \\
6239.698\end{array}$ & $\begin{array}{l}16076.671 \\
16041.580 \\
16029.889 \\
16029.262 \\
16021.984\end{array}$ & $\begin{array}{l}2 \\
2 h \\
3 c \\
8 \\
20\end{array}$ & $\begin{array}{l}1 \\
1\end{array}$ & 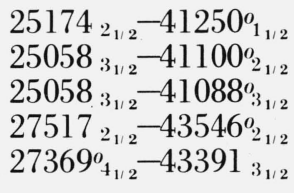 \\
\hline $\begin{array}{l}6244.178 \\
6248.971 \\
6255.807 \\
6266.102 \\
6267.598\end{array}$ & $\begin{array}{l}16010.489 \\
15998.209 \\
15980.728 \\
15954.472 \\
15950.664\end{array}$ & $\begin{array}{l}600 c w \\
15 \\
5 \\
2 w \\
2 w\end{array}$ & $\begin{array}{c}50 c \\
1 \\
1\end{array}$ & $\begin{array}{l}23588^{o} o_{1 / 2}-39598_{2_{1 / 2}} \\
28151^{o} o_{1_{1 / 2}}-44149^{o_{1 / 2}} \\
15624_{2_{1 / 2}}-31605^{o} o_{3_{1 / 2}} \\
26950_{3_{1 / 2}}-42905_{2_{1 / 2}}^{o_{1 / 2}} \\
14679_{0_{1 / 2}}-30630^{o}{ }_{1_{1 / 2}}\end{array}$ \\
\hline $\begin{array}{l}6274.014 \\
6275.425 \\
6277.250 \\
6287.962 \\
6288.358\end{array}$ & $\begin{array}{l}15934.352 \\
15930.769 \\
15926.138 \\
15899.007 \\
15898.006\end{array}$ & $\begin{array}{l}1 \\
4 \\
2 c w \\
4 c w \\
1 h\end{array}$ & & $\begin{array}{l}27971_{4_{1 / 2}}-43905^{o} o_{3_{1 / 2}} \\
25598_{2_{1 / 2}}-41529^{o} o_{3_{1 / 2}} \\
25174_{2_{1 / 2}}-41100^{o} o_{2_{1 / 2}} \\
26917_{5_{1 / 2}}-42817^{o} o_{t_{1 / 2}} \\
27274_{6_{1 / 2}}-43172_{5_{1 / 2}}^{o}\end{array}$ \\
\hline $\begin{array}{l}6290.397 \\
6292.239 \\
6296.423 \\
6296.765 \\
6304.048\end{array}$ & $\begin{array}{l}15892.852 \\
15888.200 \\
15877.642 \\
15876.780 \\
15858.438\end{array}$ & $\begin{array}{l}5 \\
1 h \\
2 \\
6 h \\
6\end{array}$ & & 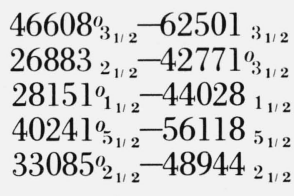 \\
\hline $\begin{array}{l}6305.028 \\
6306.260 \\
6310.420 \\
6312.179 \\
6319.033\end{array}$ & $\begin{array}{l}15855.973 \\
15852.875 \\
15842.425 \\
15838.010 \\
15820.831\end{array}$ & $\begin{array}{c}30 \\
8 \\
40 c \\
100 \\
1\end{array}$ & $\begin{array}{l}3 \\
3 c \\
10\end{array}$ & $\begin{array}{l}27940_{2_{1 / 2}}^{o}-43796_{2_{1 / 2}} \\
28296_{0_{1 / 2}}^{o}-44149_{0_{1 / 2}}^{o} \\
164155_{2_{1 / 2}}-32258^{o} o_{1 / 2} \\
16415{ }_{2_{1 / 2}}-32253_{1_{1 / 2}}^{o} \\
26950_{3_{1 / 2}}^{o}-42771_{3_{1 / 2}}^{o}\end{array}$ \\
\hline $\begin{array}{l}6321.099 \\
6326.116 \\
6347.458 \\
6354.632 \\
6354.860\end{array}$ & $\begin{array}{l}15815.660 \\
15803.118 \\
15749.983 \\
15732.203 \\
15731.638\end{array}$ & $\begin{array}{c}1 \\
1 \\
1 \\
50 \mathrm{cw} \\
100\end{array}$ & $\begin{array}{l}2 c \\
10\end{array}$ & $\begin{array}{l}152988_{3_{1 / 2}}-31114^{o}{ }_{4_{1 / 2}} \\
17282_{3_{1 / 2}}-33085^{o} o_{2_{1 / 2}} \\
27517_{2_{1 / 2}}-43267^{o} o_{3_{1 / 2}} \\
28296^{o} o_{1 / 2}-44028_{1_{1 / 2}} \\
27660_{3_{1 / 2}}^{o}-43391_{3_{1 / 2}}^{o}\end{array}$ \\
\hline $\begin{array}{l}6356.732 \\
6369.313 \\
6380.889 \\
6382.724 \\
6388.426\end{array}$ & $\begin{array}{l}15727.005 \\
15695.941 \\
15667.466 \\
15662.962 \\
15648.982\end{array}$ & $\begin{array}{c}100 \\
4 \\
5 c \\
3 \\
30 \mathrm{cw}\end{array}$ & 10 & 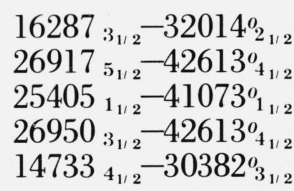 \\
\hline
\end{tabular}


TABLE 3. Classified lines of $\mathrm{Tc} \mathrm{I}$

\begin{tabular}{|c|c|c|c|c|}
\hline \multirow{2}{*}{$\begin{array}{c}\text { Wavelength } \\
\text { A }\end{array}$} & \multirow{2}{*}{$\begin{array}{l}\text { Wavenumber } \\
\mathrm{cm}^{-1}\end{array}$} & \multicolumn{2}{|c|}{ Intensity } & \multirow{2}{*}{$\begin{array}{l}\text { Energy levels } \\
\mathrm{cm}^{-1}\end{array}$} \\
\hline & & Arc & Spark & \\
\hline 6389.871 & 15645.44 & 80 & 6 & $28151^{o}{ }_{1_{1 / 2}}-43796_{2_{1 / 2}}$ \\
\hline 6392.408 & 15639.23 & $30 c$ & $2 c$ & $16287_{3_{1 / 2}}-31927^{0}{ }_{2_{1 / 2}}$ \\
\hline 6392.716 & 15638.48 & 3 & & $29233_{3_{1 / 2}}-44872_{2_{1 / 2}}^{0}$ \\
\hline 6408.830 & 15599.16 & 100 & 8 & $16415_{2_{1 / 2}}-32014^{0} o_{2_{1 / 2}}$ \\
\hline 6419.353 & 15573.58 & 2 & & $19702_{1_{1 / 2}}-35276_{0_{1 / 2}}^{0}$ \\
\hline 6432.774 & 15541.09 & 5 & & $27971_{4_{1 / 2}}-43512^{o}{ }_{5_{1 / 2}}$ \\
\hline 6445.100 & 15511.37 & $30 \mathrm{cw}$ & $1 \mathrm{cw}$ & $16415_{2_{1 / 2}}^{T_{1 / 2}}-31927_{2_{1 / 2}}^{0}$ \\
\hline 6449.026 & 15501.93 & 4 & & $25598_{2_{1 / 2}}-41100_{2_{1 / 2}}^{o}$ \\
\hline 6453.925 & 15490.16 & 3 & & $25598_{2_{1 / 2}}-41088_{3_{1 / 2}}^{o}$ \\
\hline 6455.896 & 15485.43 & 1000 & 30 & $27369^{\circ}{ }_{4_{1 / 2}}-428555_{4_{1 / 2}}$ \\
\hline 6460.338 & 15474.79 & $5 h$ & & $25598_{2_{1 / 2}}-41073^{o}{ }_{1_{1 / 2}}$ \\
\hline 6461.928 & 15470.98 & $600 c$ & $20 c$ & $16133_{4_{1 / 2}}-31605^{0}{ }_{3_{1 / 2}}$ \\
\hline 6467.570 & 15457.48 & $2 h$ & & $26788_{2_{1 / 2}}-42246_{3_{1 / 2}}^{0}$ \\
\hline 6470.270 & 15451.03 & 100 & 6 & $27940^{\circ}{ }_{2_{1 / 2}}^{-1 / 2}-43391_{3_{1 / 2}}$ \\
\hline 6479.620 & 15428.74 & $1 h$ & & $26788_{2_{1 / 2}}-42217^{0}{ }_{4_{1 / 2}}$ \\
\hline 6491.676 & 15400.08 & $200 \mathrm{cw}$ & 5 & $14733_{4_{1 / 2}}-30133^{o}{ }_{4_{1 / 2}}$ \\
\hline 6496.606 & 15388.40 & 10 & & $38556^{0}{ }_{1_{1 / 2}}-53945_{2_{1 / 2}}$ \\
\hline 6496.606 & 15388.40 & 10 & & $27517_{2_{1 / 2}}-42905_{2_{1 / 2}}^{0}$ \\
\hline 6515.160 & 15344.57 & 20 & & $25058_{3_{1 / 2}}-40403^{o}{ }_{4_{1 / 2}}$ \\
\hline 6515.660 & 15343.40 & $2 h$ & & $26950_{3_{1 / 2}}-42294_{2_{1 / 2}}^{0}$ \\
\hline 6516.192 & 15342.14 & 2 & & $29233_{3_{1 / 2}}-44576_{4_{1 / 2}}^{o}$ \\
\hline 6519.740 & 15333.79 & 1 & & $26883_{2_{1 / 2}}-42217^{0}{ }_{4_{1 / 2}}$ \\
\hline 6526.817 & 15317.17 & 200 & 20 & $16287_{3_{1 / 2}}-31605_{3_{1 / 2}}^{0}$ \\
\hline 6528.038 & 15314.30 & $5 c$ & & $26788_{2_{1 / 2}}-42102_{2_{1 / 2}}^{o}$ \\
\hline 6532.494 & 15303.86 & 2 & & $29233_{3_{1 / 2}}-44537_{2_{1 / 2}}^{0}$ \\
\hline 6534.063 & 15300.18 & 6 & & $29539_{4_{1 / 2}}-44839^{o_{3_{1 / 2}}}$ \\
\hline 6534.659 & 15298.79 & 10 & & $28839_{5_{1 / 2}}-44138_{5_{1 / 2}}$ \\
\hline 6535.947 & 15295.77 & $3 h$ & & $27971_{4_{1 / 2}}-43267^{0} 0_{3_{1 / 2}}$ \\
\hline 6536.633 & 15294.17 & 5 & & $27971_{4_{1 / 2}}-43265^{o}{ }_{4_{1 / 2}}$ \\
\hline 6537.425 & 15292.31 & $2 c$ & & $17328_{4_{1 / 2}}-32620_{3_{1 / 2}}^{0}$ \\
\hline 6563.197 & 15232.26 & 4 & & $25174_{2_{1 / 2}}-40406_{3_{1 / 2}}^{o_{1}}$ \\
\hline 6564.004 & 15230.39 & $50 w$ & & $15298_{3_{1 / 2}}-30528_{2_{1 / 2}}$ \\
\hline 6568.820 & 15219.23 & $2 h$ & & $34515_{2_{1 / 2}}^{0}-49735_{1_{1 / 2}}$ \\
\hline 6576.820 & 15200.71 & $50 w l$ & & $27274_{6_{1 / 2}}-42474_{6_{1 / 2}}^{o}$ \\
\hline 6576.820 & 15200.71 & $50 w l$ & & $27971_{41 / 2}-43172_{5_{1 / 2}}^{o}$ \\
\hline 6579.243 & 15195.12 & 150 & & $27660_{3_{1 / 2}}^{o}-42855_{4_{1 / 2}}$ \\
\hline 6581.747 & 15189.33 & 8 & & $16415_{2_{1 / 2}}-31605_{3_{1 / 2}}^{0}$ \\
\hline 6586.147 & 15179.19 & 3 & & $27517_{2_{1 / 2}}-42696_{2_{1 / 2}}^{0}$ \\
\hline 6590.039 & 15170.22 & 15 & & $27274_{6_{1 / 2}}-42444_{5_{1 / 2}}^{0}$ \\
\hline 6598.037 & 15151.83 & 10 & & $26950_{3_{1 / 2}}-42102_{2_{1 / 2}}^{o}$ \\
\hline 6609.746 & 15124.99 & $3 h s$ & & $25174_{2_{1 / 2}}-40299_{2_{1 / 2}}^{o}$ \\
\hline 6614.304 & 15114.57 & 3 & & $26917_{5_{1 / 2}}-42032^{o}{ }_{5_{1 / 2}}$ \\
\hline 6618.080 & 15105.95 & 2 & & $27971_{4_{1 / 2}}-43077_{3_{1 / 2}}^{0}$ \\
\hline 6625.573 & 15088.86 & $500 \mathrm{cw}$ & $10 \mathrm{cw}$ & $16025_{5_{1 / 2}}-31114_{4_{1 / 2}}^{o}$ \\
\hline 6627.896 & 15083.57 & $50 c$ & $1 c$ & $15298_{3_{1 / 2}}-30382_{3_{1 / 2}}^{o}$ \\
\hline 6646.143 & 15042.16 & 1 & & $26950_{3_{1 / 2}}-41993_{3_{1 / 2}}^{o}$ \\
\hline 6647.489 & 15039.12 & 4 & & $26883_{2_{1 / 2}}-41922_{2_{1 / 2}}^{0}$ \\
\hline 6648.383 & 15037.09 & 30 & 1 & $29539_{4_{1 / 2}}-44576_{4_{1 / 2}}^{0}$ \\
\hline 6650.177 & 15033.04 & 2 & & $29233_{3_{1 / 2}}-44266_{3_{1 / 2}}^{0}$ \\
\hline 6656.906 & 15017.84 & 2 & & $28839_{5_{1 / 2}}-43857^{0}{ }_{6_{1 / 2}}$ \\
\hline
\end{tabular}

602 
TABLE 3. Classified lines of T $\mathrm{T}$

\begin{tabular}{|c|c|c|c|c|}
\hline \multirow{2}{*}{$\begin{array}{c}\text { Wavelength } \\
\text { A }\end{array}$} & \multirow{2}{*}{$\begin{array}{l}\text { Wavenumber } \\
\mathrm{cm}^{-1}\end{array}$} & \multicolumn{2}{|c|}{ Intensity } & \multirow{2}{*}{$\begin{array}{l}\text { Energy levels } \\
\mathrm{cm}^{-1}\end{array}$} \\
\hline & & Arc & Spark & \\
\hline 6661.433 & 15007.64 & 5 & & $29233_{3_{1 / 2}}-44241_{2_{1 / 2}}^{o}$ \\
\hline 6662.015 & 15006.33 & 20 & & $15624_{2_{1 / 2}}-30630^{\circ}{ }_{1_{1 / 2}}^{-1 / 2}$ \\
\hline 6664.722 & 15000.23 & 2 & & $26788_{2_{1 / 2}}^{-1 / 2}-41788^{\circ}{ }_{1_{1 / 2}}$ \\
\hline 6673.656 & 14980.15 & $300 c$ & $5 c$ & $16133_{4_{1 / 2}}^{-1 / 2}-31114^{o} o_{4_{1 / 2}}$ \\
\hline 6687.099 & 14950.04 & 100 & 1 & $2572_{4_{1 / 2}}-17522^{\circ}{ }_{4_{1 / 2}}$ \\
\hline 6699.464 & 14922.44 & 2 & & $25174_{2_{1 / 2}}-40096^{0}{ }_{1_{1 / 2}}$ \\
\hline 6701.142 & 14918.71 & 5 & & $15770_{1_{1 / 2}}^{-1 / 2}-30689^{\circ} 0_{0_{1 / 2}}$ \\
\hline 6707.478 & 14904.61 & 8 & & $15624_{2_{1 / 2}}-30528_{2_{1 / 2}}$ \\
\hline 6710.202 & 14898.56 & 10 & & $13252_{2_{1 / 2}}^{-1 / 2}-28151_{1_{1 / 2}}^{o-1 / 2}$ \\
\hline 6712.512 & 14893.44 & 4 & & $25405_{1_{1 / 2}}^{-1 / 2}-40299_{2_{1 / 2}}$ \\
\hline 6718.327 & 14880.55 & $1 h$ & & $26883_{2_{1 / 2}}-41764_{2_{1 / 2}}^{o}$ \\
\hline 6721.629 & 14873.24 & 3 & & $29233_{3_{1 / 2}}^{-1 / 2}-44107_{2_{1 / 2}}^{-1 / 2}$ \\
\hline 6731.187 & 14852.12 & 50 & & $30133^{o}{ }_{4_{1 / 2}}-44985_{3_{1 / 2}}$ \\
\hline 6733.847 & 14846.25 & 7 & & $268833_{2_{1 / 2}}-41729_{3_{1 / 2}}$ \\
\hline 6738.066 & 14836.95 & 1 & & $26788_{2_{1 / 2}}-41625^{0}{ }_{1_{1 / 2}}$ \\
\hline 6742.901 & 14826.31 & 5 & & $16287_{3_{1 / 2}}-31114_{4_{1 / 2}}^{o}$ \\
\hline 6745.042 & 14821.61 & 6 & & $34515_{2_{1 / 2}}^{\circ 1 / 2}-49337_{2_{1 / 2}}$ \\
\hline 6747.530 & 14816.14 & $7 h$ & & $30060_{2_{1 / 2}}-44876_{1_{1 / 2}}^{0}$ \\
\hline 6748.838 & 14813.27 & 5 & & $26950_{3_{1 / 2}}^{-1 / 2}-41764_{2_{1 / 2}}^{0^{1 / 2}}$ \\
\hline 6753.399 & 14803.27 & 10 & & $25174_{2_{1 / 2}}-39977^{0} 0_{2_{1 / 2}}$ \\
\hline 6758.326 & 14792.48 & 20 & & $34942^{o} o_{1_{1 / 2}}-49735_{1_{1 / 2}}$ \\
\hline 6761.469 & 14785.60 & 30 & 1 & $26917_{5_{1 / 2}}-41703^{\circ}{ }_{4_{1 / 2}}$ \\
\hline 6764.101 & 14779.85 & 2 & & $47819^{\circ}{ }_{4_{1 / 2}}-62599_{4_{1 / 2}}$ \\
\hline 6764.574 & 14778.81 & 5 & & $26950_{3_{1 / 2}}-41729_{3_{1 / 2}}^{o}$ \\
\hline 6764.758 & 14778.41 & 8 & & $30060_{2_{1 / 2}}-44839^{o} o_{1 / 2}$ \\
\hline 6767.183 & 14773.12 & 2 & & $29233_{3_{1 / 2}}-44007_{2_{1 / 2}}^{o_{2}}$ \\
\hline 6773.899 & 14758.47 & 4 & & $15770_{1_{1 / 2}}-30528_{2_{1 / 2}}^{-1 / 2}$ \\
\hline 6776.580 & 14752.63 & 8 & & $26950_{3_{1 / 2}}-41703_{4_{1 / 2}}^{o}$ \\
\hline 6786.003 & 14732.14 & 80 & 2 & $17282_{3_{1 / 2}}-32014_{2_{1 / 2}}^{0}$ \\
\hline 6786.965 & 14730.06 & 10 & & $34515_{2_{1 / 2}}^{o}-49245_{3_{1 / 2}}$ \\
\hline 6787.951 & 14727.92 & 2 & & $29539_{4_{1 / 2}}-44266_{3_{1 / 2}}^{0}$ \\
\hline 6795.564 & 14711.42 & 2 & & $20231_{1_{1 / 2}}-34942^{o}{ }_{1_{1 / 2}}$ \\
\hline 6798.629 & 14704.79 & 70 & 2 & $25058_{3_{1 / 2}}-39763^{\circ}{ }_{4_{1 / 2}}$ \\
\hline 6800.793 & 14700.11 & 2 & & $27517_{2_{1 / 2}}-42217^{\circ} 4_{1 / 2}$ \\
\hline 6805.049 & 14690.91 & 5 & & $25405_{1_{1 / 2}}-40096^{0}{ }_{1_{1 / 2}}$ \\
\hline 6805.942 & 14688.98 & 15 & & $30382_{3_{1 / 2}}^{o}-45071_{2_{1 / 2}}$ \\
\hline 6813.340 & 14673.04 & 5 & & $28839_{5_{1 / 2}}-43512^{o} \sigma_{5_{1 / 2}}$ \\
\hline 6818.126 & 14662.74 & 10 & & $26917_{5_{1 / 2}}-41580_{5_{1 / 2}}^{o}$ \\
\hline 6826.146 & 14645.51 & 5 & & $26883_{2_{1 / 2}}-41529^{\circ}{ }_{3_{1 / 2}}$ \\
\hline 6826.686 & 14644.35 & 5 & & $17282_{3_{1 / 2}}-31927_{2_{1 / 2}}^{\circ}$ \\
\hline 6830.007 & 14637.23 & 8 & & $30630^{o}{ }_{1_{1 / 2}}-45267_{1_{1 / 2}}$ \\
\hline 6833.736 & 14629.24 & 3 & & $27971_{4_{1 / 2}}-42600^{\circ} 5_{5_{1 / 2}}$ \\
\hline 6837.906 & 14620.32 & $4 h w$ & & $19895_{2_{1 / 2}}-34515_{2_{1 / 2}}^{\circ}$ \\
\hline 6837.906 & 14620.32 & $4 h w$ & & $24564_{5_{1 / 2}}-39185_{4_{1 / 2}}$ \\
\hline 6856.902 & 14579.82 & 60 & 1 & $30528_{2_{1 / 2}}-45108_{1_{1 / 2}}$ \\
\hline 6860.726 & 14571.69 & 30 & 1 & $25405_{1_{1 / 2}}-39977_{2_{1 / 2}}^{o}$ \\
\hline 6866.677 & 14559.06 & 25 & & $30630^{\circ}{ }_{1_{1 / 2}}-45189_{0_{1 / 2}}$ \\
\hline 6874.626 & 14542.23 & 10 & & $30528_{2_{1^{\prime} / 2}}-45071_{2_{1 / 2}}$ \\
\hline 6894.386 & 14500.55 & 20 & & $30689_{0_{1 / 2}}^{-1 / 2}-45189_{0_{1 / 2}}^{-1 / 2}$ \\
\hline 6901.620 & 14485.35 & 2 & & $39459_{2_{1 / 2}}^{o}-53945_{2_{1 / 2}}$ \\
\hline
\end{tabular}


TABLE 3. Classified lines of Tc I

\begin{tabular}{|c|c|c|c|c|}
\hline \multirow{2}{*}{$\begin{array}{c}\text { Wavelength } \\
\text { A }\end{array}$} & \multirow{2}{*}{$\begin{array}{l}\text { Wavenumber } \\
\mathrm{cm}^{-1}\end{array}$} & \multicolumn{2}{|c|}{ Intensity } & \multirow{2}{*}{$\underset{\mathrm{cm}^{-1}}{\text { Energy levels }}$} \\
\hline & & Arc & Snark & \\
\hline $\begin{array}{l}6905.117 \\
6907.514 \\
6924.055 \\
6928.934 \\
6931.795\end{array}$ & $\begin{array}{l}14478.02 \\
14472.99 \\
14438.42 \\
14428.25 \\
14422.29\end{array}$ & $\begin{array}{l}10 \\
30 \\
15 \\
3 h \\
30\end{array}$ & & 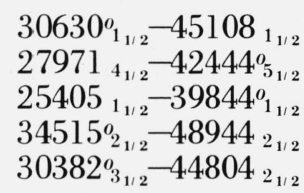 \\
\hline $\begin{array}{l}6933.236 \\
6939.892 \\
6944.988 \\
6945.597 \\
6949.320\end{array}$ & $\begin{array}{l}14419.30 \\
14405.47 \\
14394.90 \\
14393.64 \\
14385.92\end{array}$ & $\begin{array}{r}30 \\
4 \\
2 \\
15 \\
7 h\end{array}$ & & $\begin{array}{l}205233_{2_{1 / 2}}-34942^{o}{ }_{1_{1 / 2}} \\
27517_{2_{1 / 2}}-41922^{o}{ }_{2_{1 / 2}} \\
34942^{o}{ }_{1_{1 / 2}}-49337_{1_{1 / 2}} \\
25174{ }_{{ }_{1 / 2}}-39567^{\circ}{ }_{3_{1 / 2}} \\
48213^{o}{ }_{4_{1 / 2}}-62599_{4_{1 / 2}}\end{array}$ \\
\hline $\begin{array}{l}6952.625 \\
6958.593 \\
6968.151 \\
6975.067 \\
6980.153\end{array}$ & $\begin{array}{l}14379.09 \\
14366.75 \\
14347.05 \\
14332.82 \\
14322.38\end{array}$ & $\begin{array}{r}3 \\
4 \\
30 \\
7 \\
10\end{array}$ & & $\begin{array}{l}25598_{2_{1 / 2}}-39977^{0} o_{2_{1 / 2}} \\
29539_{4_{1 / 2}}-43905^{o} o_{3_{1 / 2}} \\
23265_{3_{1 / 2}}^{o}-37612_{3_{1 / 2}} \\
28839_{5_{1 / 2}}-43172^{o} 5_{5_{1 / 2}} \\
17282_{3_{1 / 2}}-31605^{o}{ }_{3_{1 / 2}}\end{array}$ \\
\hline $\begin{array}{l}6985.402 \\
6985.402 \\
6990.300 \\
6998.037 \\
6998.653\end{array}$ & $\begin{array}{l}14311.62 \\
14311.62 \\
14301.59 \\
14285.78 \\
14284.52\end{array}$ & $\begin{array}{r}30 \\
30 \\
40 \\
8 \\
15\end{array}$ & & 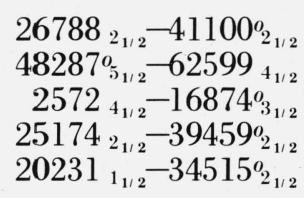 \\
\hline $\begin{array}{l}7002.375 \\
7004.781 \\
7006.293 \\
7011.448 \\
7014.108\end{array}$ & $\begin{array}{l}14276.93 \\
14272.02 \\
14268.94 \\
14258.45 \\
14253.05\end{array}$ & $\begin{array}{c}150 \\
6 \\
20 c \\
2 \\
10 c\end{array}$ & & $\begin{array}{r}17328_{4_{1 / 2}}-31605^{o} o_{3_{1 / 2}} \\
3250_{3_{1 / 2}}-17522^{3}{ }_{4_{1 / 2}} \\
34515^{o_{1 / 2}}-48784^{a_{1 / 2}}{ }_{3_{1 / 2}} \\
28839_{5_{1 / 2}}-43097^{\circ}{ }_{4_{1 / 2}} \\
30060_{2_{1 / 2}}-44313^{o} o_{1 / 2}\end{array}$ \\
\hline $\begin{array}{l}7016.574 \\
7020.025 \\
7029.723 \\
7032.894 \\
7054.800\end{array}$ & $\begin{array}{l}14248.04 \\
14241.03 \\
14221.39 \\
14214.97 \\
14170.83\end{array}$ & $\begin{array}{r}100 \\
20 \\
8 \\
10 \\
10\end{array}$ & & $\begin{array}{l}161333_{4_{1 / 2}}-30382^{o}{ }_{3_{1 / 2}} \\
16287_{3_{1 / 2}}-30528^{o}{ }_{2_{1 / 2}} \\
41896^{o}{ }_{4_{1 / 2}}-56118_{5_{1 / 2}}^{a_{1 / 2}} \\
164155_{2_{1 / 2}}-30630^{o}{ }_{1_{1 / 2}} \\
254055_{1_{1 / 2}}-39576^{o} o_{1_{1 / 2}}\end{array}$ \\
\hline $\begin{array}{l}7061.633 \\
7065.596 \\
7071.442 \\
7076.770 \\
7083.599\end{array}$ & $\begin{array}{l}14157.12 \\
14149.18 \\
14137.48 \\
14126.84 \\
14113.22\end{array}$ & $\begin{array}{r}7 \\
10 \\
30 \\
15 \\
15\end{array}$ & & $\begin{array}{l}23455_{2_{1 / 2}}^{o}-37612_{3_{1 / 2}} \\
26950_{3_{1 / 2}}-41100^{o}{ }_{2_{1 / 2}} \\
26950_{3_{1 / 2}}-41088^{\circ}{ }_{3_{1 / 2}} \\
14169_{1_{1 / 2}}-28296^{\circ} 0_{1_{1 / 2}} \\
16415_{2_{1 / 2}}-30528_{2_{1 / 2}}\end{array}$ \\
\hline $\begin{array}{l}7084.871 \\
7086.180 \\
7093.120 \\
7097.242 \\
7109.813\end{array}$ & $\begin{array}{l}14110.69 \\
14108.08 \\
14094.28 \\
14086.09 \\
14061.19\end{array}$ & $\begin{array}{c}1 \\
560 c w \\
60 \\
3 \\
8\end{array}$ & & $\begin{array}{l}295399_{4_{1 / 2}}-43649^{o}{ }_{4_{1 / 2}} \\
160255_{5_{1 / 2}}-30133^{o}{ }_{4_{1 / 2}} \\
16287{ }_{3_{1 / 2}}-30382^{o}{ }_{3_{1 / 2}} \\
17328{ }_{4_{1 / 2}}-31414^{o}{ }_{3_{1 / 2}} \\
27971_{4_{1 / 2}}-42032^{o}{ }_{5_{1 / 2}}\end{array}$ \\
\hline $\begin{array}{l}7115.781 \\
7117.170 \\
7123.974 \\
7124.784 \\
7134.829\end{array}$ & $\begin{array}{l}14049.39 \\
14046.65 \\
14033.24 \\
14031.64 \\
14011.89\end{array}$ & $\begin{array}{r}8 \\
4 \\
4 \\
30 \\
1\end{array}$ & & $\begin{array}{l}30060_{2_{1 / 2}}-44110^{o} a_{1_{1 / 2}} \\
30060_{2_{1 / 2}}-44107^{\circ} a_{2_{1 / 2}} \\
29233_{3_{1 / 2}}-43267^{\circ}{ }_{3_{1 / 2}} \\
29233_{3_{1 / 2}}-43265^{\circ}{ }_{4_{1 / 2}} \\
27517_{2_{1 / 2}}-41529^{\circ}{ }_{3_{1 / 2}}\end{array}$ \\
\hline $\begin{array}{l}7140.187 \\
7141.286 \\
7144.678 \\
7156.874 \\
7157.626\end{array}$ & $\begin{array}{l}14001.37 \\
13999.22 \\
13992.57 \\
13968.73 \\
13967.26\end{array}$ & $\begin{array}{r}3 \\
200 \\
15 \\
20 \\
200 c\end{array}$ & & $\begin{array}{l}34942^{o}{ }_{1_{1 / 2}}-48944_{{ }_{1 / 2}} \\
16133_{4_{1 / 2}}-30133^{o}{ }_{4_{1 / 2}} \\
20523_{2_{1 / 2}}-34515^{o}{ }_{2_{1 / 2}} \\
30133^{o}{ }_{4_{1 / 2}}-44102^{{ }_{3}}{ }_{3_{1 / 2}} \\
17146_{5_{1 / 2}}-31114^{o}{ }_{4_{1 / 2}}\end{array}$ \\
\hline
\end{tabular}


TABLE 3. Classified lines of TC I

\begin{tabular}{|c|c|c|c|c|}
\hline \multirow{2}{*}{$\begin{array}{l}\text { Wavelength } \\
\text { A }\end{array}$} & \multirow{2}{*}{$\begin{array}{l}\text { Wavenumber } \\
\mathrm{cm}^{-1}\end{array}$} & \multicolumn{2}{|c|}{ Intensity } & \multirow{2}{*}{$\begin{array}{l}\text { Energy levels } \\
\mathrm{cm}^{-1}\end{array}$} \\
\hline & & Arc & Spark & \\
\hline $\begin{array}{l}7179.043 \\
7191.591 \\
7194.656 \\
7207.026 \\
7220.543\end{array}$ & $\begin{array}{l}13925.59 \\
13901.29 \\
13895.37 \\
13871.52 \\
13845.55\end{array}$ & $\begin{array}{r}10 \\
3 \\
2 \\
8 \\
2\end{array}$ & & $\begin{array}{l}27971_{4_{1 / 2}}-41896^{o}{ }_{4_{1 / 2}} \\
42217^{0}{ }_{4_{1 / 2}}-561188_{5_{1 / 2}} \\
48142^{o} o_{2_{1 / 2}}-62038_{3_{1 / 2}} \\
31114^{o} o_{4_{1 / 2}}-44985_{3_{1 / 2}} \\
16287_{3_{1 / 2}}-30133_{4_{1 / 2}}^{o}\end{array}$ \\
\hline $\begin{array}{l}7251.685 \\
7256.089 \\
7264.840 \\
7283.164 \\
7296.248\end{array}$ & $\begin{array}{l}13786.09 \\
13777.73 \\
13761.13 \\
13726.51 \\
13701.89\end{array}$ & $\begin{array}{l}15 \\
70 \\
3 \\
15 \\
5 w d\end{array}$ & & $\begin{array}{l}17328_{4_{1 / 2}}-31114^{o}{ }_{4_{1 / 2}} \\
19702_{1_{1 / 2}}-33480^{o} 0_{0_{1 / 2}} \\
28839_{5_{1 / 2}}-42600^{o} o_{5_{1 / 2}} \\
29539_{4_{1 / 2}}-43265^{o}{ }_{4_{1 / 2}} \\
31406_{2_{1 / 2}}^{o}-45108_{1_{1 / 2}}^{o}\end{array}$ \\
\hline $\begin{array}{l}7320.300 \\
7322.382 \\
7329.148 \\
7331.895 \\
7338.202\end{array}$ & $\begin{array}{l}13656.88 \\
13652.99 \\
13640.39 \\
13635.28 \\
13623.56\end{array}$ & $\begin{array}{r}5 w \\
100 \\
80 \\
5 \\
50\end{array}$ & & $\begin{array}{r}31414_{3_{1 / 2}}^{o}-45071_{2_{1 / 2}} \\
19827_{0_{1 / 2}}-33480^{o} o_{0_{1 / 2}} \\
19702_{1_{1 / 2}}-33343^{o} o_{1 / 2} \\
28839_{5_{1 / 2}}-42474^{o} o_{b_{1 / 2}} \\
3250_{3_{1 / 2}}-16874^{o} o_{3_{1 / 2}}\end{array}$ \\
\hline $\begin{array}{l}7340.134 \\
7341.852 \\
7345.819 \\
7360.063 \\
7362.460\end{array}$ & $\begin{array}{l}13619.97 \\
13616.79 \\
13609.43 \\
13583.09 \\
13578.67\end{array}$ & $\begin{array}{c}4 \\
2 \\
4 \\
10 \\
5 w\end{array}$ & & $\begin{array}{l}48881_{4_{1 / 2}}^{o}-62501_{3_{1 / 2}} \\
14679_{0_{1 / 2}}-28296^{\circ} o_{1 / 2} \\
27971_{4_{1 / 2}}-41580^{\circ} \sigma_{5_{1 / 2}} \\
29233_{3_{1 / 2}}-42817^{\circ}{ }_{4_{1 / 2}} \\
31406_{2_{1 / 2}}^{o}-44985_{3_{1 / 2}}\end{array}$ \\
\hline $\begin{array}{l}7366.336 \\
7395.066 \\
7402.619 \\
7405.363 \\
7421.120\end{array}$ & $\begin{array}{l}13571.53 \\
13518.80 \\
13505.01 \\
13500.00 \\
13471.34\end{array}$ & $\begin{array}{l}40 c w \\
50 \\
100 c w \\
200 \\
8\end{array}$ & & $\begin{array}{l}31414^{o} o_{3_{1 / 2}}-449855_{3_{1 / 2}} \\
30630^{o}{ }_{1_{1 / 2}}-44149_{0_{1 / 2}} \\
31414^{o}{ }_{3_{1 / 2}}-44919_{4_{1 / 2}} \\
30528_{2_{1 / 2}}^{o}-44028_{1_{1 / 2}} \\
14679_{0_{1 / 2}}-28151_{1_{1 / 2}}^{o}\end{array}$ \\
\hline $\begin{array}{l}7424.011 \\
7427.154 \\
7197 . \mathrm{s}\end{array}$ & $\begin{array}{l}13466.09 \\
13460.40 \\
19450.25\end{array}$ & $\begin{array}{r}20 \\
60 \\
? \\
?\end{array}$ & & 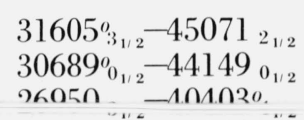 \\
\hline $\begin{array}{l}7434.122 \\
7452.496\end{array}$ & $\begin{array}{l}13447.78 \\
13414.62\end{array}$ & $\begin{array}{l}150 \\
600\end{array}$ & & $\begin{array}{l}19895_{2_{1 / 2}}-33343^{o}{ }_{1_{1 / 2}} \\
30382_{3_{1 / 2}}^{o}-43796_{2_{1 / 2}}^{o}\end{array}$ \\
\hline $\begin{array}{l}7461.594 \\
7470.148 \\
7471.776 \\
7494.288 \\
7512.280\end{array}$ & $\begin{array}{l}13398.27 \\
13382.93 \\
13380.01 \\
13339.82 \\
13307.87\end{array}$ & $\begin{array}{r}60 \\
50 \\
20 \\
6 \\
3\end{array}$ & & $\begin{array}{l}30630^{o}{ }_{1_{1 / 2}}-44028_{1_{1 / 2}} \\
19702_{1_{1 / 2}}-33085^{o}{ }_{2_{1 / 2}} \\
29233_{3_{1 / 2}}-42613^{o}{ }_{4_{1 / 2}} \\
30689^{o} 0_{1 / 2}-44028_{1_{1 / 2}}^{o_{1 / 2}}-40096^{o}{ }_{1_{1 / 2}}\end{array}$ \\
\hline $\begin{array}{l}7534.952 \\
7540.265 \\
7543.391 \\
7545.510 \\
7547.268\end{array}$ & $\begin{array}{l}13267.83 \\
13258.48 \\
13252.98 \\
13249.26 \\
13246.18\end{array}$ & $\begin{array}{r}80 \\
800 \\
80 \\
30 \\
5\end{array}$ & & $\begin{array}{l}30528_{2_{1 / 2}}^{0}-43796_{2_{1 / 2}} \\
30133_{4_{1 / 2}}^{o}-43391_{3_{1 / 2}} \\
32014_{2_{1 / 2}}^{o}-45267_{1_{1 / 2}}^{o} \\
20231_{1_{1 / 2}}-33480^{o} 0_{1 / 2} \\
17282_{3_{1 / 2}}-30528_{2_{1 / 2}}^{o}\end{array}$ \\
\hline $\begin{array}{l}7548.442 \\
7550.991 \\
7574.026 \\
7577.614 \\
7579.267\end{array}$ & $\begin{array}{l}13244.12 \\
13239.65 \\
13199.38 \\
13193.13 \\
13190.25\end{array}$ & $\begin{array}{c}50 \\
10 w \\
200 \\
2 \\
500\end{array}$ & & $\begin{array}{l}32253^{o}{ }_{1_{1 / 2}}-45497_{0_{1 / 2}} \\
32258^{o}{ }_{1_{1 / 2}}-45497_{0_{1 / 2}} \\
31605^{o} 3_{1 / 2}-44804_{2_{1 / 2}} \\
28839_{5_{1 / 2}}-42032_{5_{1 / 2}}^{o} \\
19895_{2_{1 / 2}}-33085_{2_{1 / 2}}^{o}\end{array}$ \\
\hline $\begin{array}{l}7583.935 \\
7583.935 \\
7586.447 \\
7588.611 \\
7593.152\end{array}$ & $\begin{array}{l}13182.13 \\
13182.13 \\
13177.77 \\
13174.01 \\
13166.13\end{array}$ & $\begin{array}{l}5 \\
5 \\
5 \\
7 \\
7\end{array}$ & & $\begin{array}{r}19903_{3_{1 / 2}}-33085_{2_{1 / 2}}^{o} \\
25058_{3_{1 / 2}}-38240^{o} o_{3_{1 / 2}} \\
3250_{3_{1 / 2}}-16428_{2_{1 / 2}}^{o} \\
3700_{2_{1 / 2}}-16874^{o} o_{3_{1 / 2}} \\
30630_{1_{1 / 2}}^{o}-43796_{2_{1 / 2}}^{o}\end{array}$ \\
\hline
\end{tabular}


TABLE 3. Classified lines of Tc I

\begin{tabular}{|c|c|c|c|c|}
\hline \multirow{2}{*}{$\begin{array}{c}\text { Wavelength } \\
\text { A }\end{array}$} & \multirow{2}{*}{$\begin{array}{c}\text { Wavenumber } \\
\mathrm{cm}^{-1}\end{array}$} & \multicolumn{2}{|c|}{ Intensity } & \multirow{2}{*}{$\underset{\mathrm{cm}^{-1}}{\text { Energy levels }}$} \\
\hline & & Arc & Spark & \\
\hline $\begin{array}{l}7624.538 \\
7631.825 \\
7634.531 \\
7635.083 \\
7657.044\end{array}$ & $\begin{array}{l}13111.93 \\
13099.42 \\
13094.77 \\
13093.83 \\
13056.27\end{array}$ & $\begin{array}{r}90 \\
4 \\
7 \\
4 \\
2\end{array}$ & & $\begin{array}{l}20231_{1_{1 / 2}}-33343^{o} o_{1 / 2} \\
17282_{3_{1 / 2}}-30382^{o_{3_{1 / 2}}} \\
32403_{0_{1 / 2}}^{o}-45497_{0_{1 / 2}}^{o_{1 / 2}} \\
32014_{2_{1 / 2}}^{o}-45108_{1_{1 / 2}}^{o} \\
32014_{2_{1 / 2}}^{o}-45071_{2_{1 / 2}}^{o}\end{array}$ \\
\hline $\begin{array}{l}7658.374 \\
7676.957 \\
7681.790 \\
7684.458 \\
7684.458\end{array}$ & $\begin{array}{l}13054.00 \\
13022.41 \\
13014.21 \\
13009.69 \\
13009.69\end{array}$ & $\begin{array}{c}30 c \\
4 \\
15 \\
100 \\
100\end{array}$ & & $\begin{array}{l}17328_{4_{1 / 2}}-30382^{o}{ }_{3_{1 / 2}} \\
21920_{2_{1 / 2}}-34942^{o}{ }_{1_{1 / 2}} \\
32253^{o}{ }_{1_{1 / 2}}-45267_{1_{1 / 2}} \\
30382^{o}{ }_{3_{1 / 2}}-43391_{3_{1 / 2}} \\
32258^{o}{ }_{1_{1 / 2}}-45267_{1_{1 / 2}}\end{array}$ \\
\hline $\begin{array}{l}7697.370 \\
7698.190 \\
7719.592 \\
7722.199 \\
7733.630\end{array}$ & $\begin{array}{l}12987.87 \\
12986.49 \\
12950.48 \\
12946.11 \\
12926.98\end{array}$ & $\begin{array}{l}500 \\
80 c w \\
2 h \\
3 \\
5 c\end{array}$ & & $\begin{array}{l}31114^{o} o_{4_{1 / 2}}-44102_{3_{1 / 2}} \\
17146_{5_{1 / 2}}-30133_{4_{1 / 2}}^{o} \\
30060_{2_{1 / 2}}-43011^{o} o_{1 / 2} \\
43172^{o} o_{5_{1 / 2}}-56118_{5_{1 / 2}}-27660^{o} o_{3_{1 / 2}}\end{array}$ \\
\hline $\begin{array}{l}7737.368 \\
7746.605 \\
7777.220 \\
7779.594 \\
7779.594\end{array}$ & $\begin{array}{l}12920.73 \\
12905.32 \\
12854.52 \\
12850.60 \\
12850.60\end{array}$ & $\begin{array}{c}50 c \\
10 \\
40 \\
6 c \\
6 c\end{array}$ & & $\begin{array}{l}17146_{5_{1 / 2}}-30067^{0}{ }_{5_{1 / 2}} \\
29539_{4_{1 / 2}}-424444^{o} 5_{1_{1 / 2}} \\
20231_{1_{1 / 2}}-33085^{o} o_{2_{1 / 2}} \\
17282_{3_{1 / 2}}-30133^{o} o_{4_{1 / 2}} \\
32258^{o} o_{1_{1 / 2}}-45108_{1_{1 / 2}}\end{array}$ \\
\hline $\begin{array}{l}7782.993 \\
7782.993 \\
7793.046 \\
7798.280 \\
7807.200\end{array}$ & $\begin{array}{l}12844.99 \\
12844.99 \\
12828.42 \\
12819.81 \\
12805.16\end{array}$ & $\begin{array}{l}15 \\
15 \\
800 \mathrm{cw} \\
60 \\
10\end{array}$ & & $\begin{array}{l}41100_{2_{1 / 2}}^{o} 53945_{2_{1 / 2}} \\
30060_{{ }_{1 / 2}}-42905^{o}{ }_{2_{1 / 2}} \\
19791_{4_{1 / 2}}-32620^{o}{ }_{{ }_{1 / 2}} \\
20523_{2_{1 / 2}}-33343^{o}{ }_{{ }_{1 / 2}} \\
17328_{4_{1 / 2}}-30133^{o}{ }_{4_{1 / 2}}\end{array}$ \\
\hline $\begin{array}{l}7816.741 \\
7817.728 \\
7854.400 \\
7856.380 \\
7858.250\end{array}$ & $\begin{array}{l}12789.53 \\
12787.92 \\
12728.21 \\
12725.00 \\
12721.97\end{array}$ & $\begin{array}{r}60 \\
800 \\
10 \\
100 \\
10\end{array}$ & & $\begin{array}{r}32014_{2_{1 / 2}}^{0}-44804_{2_{1 / 2}} \\
30067_{5_{1 / 2}}^{o}-42855_{4_{1 / 2}} \\
3700_{2_{1 / 2}}-16428_{2_{1 / 2}}^{o} \\
19895_{2_{1 / 2}}-32620^{\circ} o_{3_{1 / 2}} \\
30133_{4_{1 / 2}}^{o}-42855_{4_{1 / 2}}\end{array}$ \\
\hline $\begin{array}{l}7861.440 \\
7871.250 \\
7874.760 \\
7876.190 \\
7879.370\end{array}$ & $\begin{array}{l}12716.81 \\
12700.96 \\
12695.30 \\
12693.00 \\
12687.87\end{array}$ & $\begin{array}{c}200 \\
400 l d \\
60 c w \\
2 \\
8 c\end{array}$ & & $\begin{array}{l}19903_{3_{1 / 2}}-32620^{o} 3_{1 / 2} \\
20384_{3_{1 / 2}}-33085^{o}{ }_{2_{1 / 2}} \\
31406_{2_{1 / 2}}^{o}-44102_{3_{1 / 2}} \\
26883_{2_{1 / 2}}-39576_{1_{1 / 2}}^{o} \\
31414_{3_{1 / 2}}^{o}-44102_{3_{1 / 2}}\end{array}$ \\
\hline $\begin{array}{l}7881.630 \\
7885.980 \\
7894.750 \\
7898.567 \\
7905.729\end{array}$ & $\begin{array}{l}12684.24 \\
12677.24 \\
12663.16 \\
12657.04 \\
12645.57\end{array}$ & $\begin{array}{c}5 \\
40 c w \\
2 \\
3 \\
20 c\end{array}$ & & $\begin{array}{l}26883_{2_{1 / 2}}-39567^{\circ} o_{3_{1 / 2}} \\
22265_{2_{1 / 2}}-34942^{\circ}{ }_{1_{1 / 2}} \\
29233_{3_{1 / 2}}-41896^{o}{ }_{4_{1 / 2}} \\
20686_{2_{1 / 2}}-33343^{o}{ }_{1_{1 / 2}} \\
31503_{1_{1 / 2}}^{o}-44149_{0_{1 / 2}}^{o}\end{array}$ \\
\hline $\begin{array}{l}7911.290 \\
7920.371 \\
7949.716 \\
7958.092 \\
7965.452\end{array}$ & $\begin{array}{l}12636.68 \\
12622.19 \\
12575.60 \\
12562.37 \\
12550.76\end{array}$ & $\begin{array}{r}4 c \\
10 h \\
15 c \\
5 \\
70\end{array}$ & & $\begin{array}{l}14733_{4_{1 / 2}}-27369^{\circ}{ }_{4_{1 / 2}} \\
31406^{o}{ }_{2_{1 / 2}}-44028_{1_{1 / 2}} \\
19827^{0_{1 / 2}}-32403^{0} o_{0_{1 / 2}} \\
20523_{2_{1 / 2}}-33085^{\circ}{ }_{2_{1 / 2}} \\
32253_{1_{1 / 2}}^{o}-44804_{2_{1 / 2}}^{o}\end{array}$ \\
\hline $\begin{array}{l}7968.304 \\
7981.805 \\
7999.730 \\
8001.889 \\
8017.229\end{array}$ & $\begin{array}{l}12546.27 \\
12525.04 \\
12496.98 \\
12493.61 \\
12469.70\end{array}$ & $\begin{array}{l}50 \\
50 \mathrm{cw} \\
500 \\
10 \\
8\end{array}$ & & $\begin{array}{l}32258^{o}{ }_{1_{1 / 2}}-44804 \\
2_{1 / 2} \\
31503^{o}{ }_{1_{1 / 2}}-44028_{1_{1 / 2}} \\
31605^{o}{ }_{3_{1 / 2}}-44102_{3_{1 / 2}} \\
29539_{4_{1 / 2}}-42032^{o} 5_{5_{1 / 2}} \\
29233_{3_{1 / 2}}-41703^{o}{ }_{4_{1 / 2}}\end{array}$ \\
\hline
\end{tabular}


TABLE 3. Classified lines of $\mathrm{Tc} \mathrm{I}$

\begin{tabular}{|c|c|c|c|c|}
\hline \multirow{2}{*}{$\begin{array}{c}\text { Wavelength } \\
\text { A }\end{array}$} & \multirow{2}{*}{$\underset{\substack{\text { Wavenumber } \\
\mathrm{cm}^{-1}}}{\text {. }}$} & \multicolumn{2}{|c|}{ Intensity } & \multirow{2}{*}{$\begin{array}{l}\text { Energy levels } \\
\mathrm{cm}^{-1}\end{array}$} \\
\hline & & Arc & Spark & \\
\hline $\begin{array}{l}8029.468 \\
8042.473 \\
8045.330 \\
8068.794 \\
8073.665\end{array}$ & $\begin{array}{l}12450.70 \\
12430.56 \\
12426.15 \\
12390.01 \\
12382.54\end{array}$ & $\begin{array}{l}8 c \\
30 \\
25 \\
40 c \\
10 c\end{array}$ & & 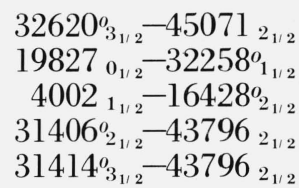 \\
\hline $\begin{array}{l}8112.776 \\
8116.919 \\
8119.869 \\
8126.557 \\
8132.651\end{array}$ & $\begin{array}{l}12322.84 \\
12316.55 \\
12312.08 \\
12301.95 \\
12292.73\end{array}$ & $\begin{array}{c}1 \\
5 \\
40 \\
200 \\
5 h\end{array}$ & & 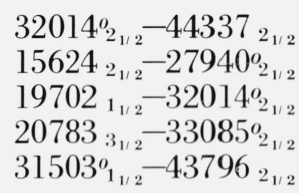 \\
\hline $\begin{array}{l}8142.630 \\
8170.558 \\
8200.050 \\
8205.276 \\
8206.495\end{array}$ & $\begin{array}{l}12277.66 \\
12235.70 \\
12191.69 \\
12183.93 \\
12182.12\end{array}$ & $\begin{array}{r}30 \\
200 \\
3 \\
150 \\
100\end{array}$ & & $\begin{array}{l}31114^{o}{ }_{4_{1 / 2}}-43391_{3_{1 / 2}} \\
20384_{3_{1 / 2}}-32620^{o}{ }_{3_{1 / 2}} \\
31605^{o}{ }_{3_{1 / 2}}-43796_{2_{1 / 2}} \\
32620^{o} 3_{3_{1 / 2}}-44804_{2_{1 / 2}} \\
33085_{2_{1 / 2}}^{o}-45267_{1_{1 / 2}}\end{array}$ \\
\hline $\begin{array}{l}8211.310 \\
8225.074 \\
8237.089 \\
8248.960 \\
8254.505\end{array}$ & $\begin{array}{l}12174.97 \\
12154.60 \\
12136.87 \\
12119.40 \\
12111.26\end{array}$ & $\begin{array}{l}150 \\
50 \\
500 \mathrm{cw} \\
40 \\
30\end{array}$ & & $\begin{array}{l}31927^{o}{ }_{2_{1 / 2}}-44102_{3_{1 / 2}} \\
33343^{o}{ }_{1_{1 / 2}}-45497_{0_{1 / 2}} \\
21206{ }_{2_{1 / 2}}-33343^{o}{ }_{1_{1 / 2}} \\
19895{ }_{2_{1 / 2}}-32014_{2_{1 / 2}}^{o} \\
199033_{3_{1 / 2}}-32014_{2_{1 / 2}}^{o}\end{array}$ \\
\hline $\begin{array}{l}8264.138 \\
8276.202 \\
8276.202 \\
8306.220 \\
8308.158\end{array}$ & $\begin{array}{l}12097.15 \\
12079.51 \\
12079.51 \\
12035.86 \\
12033.05\end{array}$ & $\begin{array}{c}10 \\
1 h \\
1 h \\
2 \\
200\end{array}$ & & $\begin{array}{l}205233_{2_{1 / 2}}-32620^{o} o_{3_{1 / 2}} \\
32253^{o} o_{1 / 2}-443333_{1_{1 / 2}} \\
32258^{o}{ }_{1_{1 / 2}}-44337^{a_{1 / 2}} \\
15624{ }_{2_{1 / 2}}-27660^{o} 3_{3_{1 / 2}} \\
21447_{1_{1 / 2}}-33480^{o} 0_{0_{1 / 2}}\end{array}$ \\
\hline $\begin{array}{l}8309.161 \\
8315.502 \\
8319.074 \\
8346.576 \\
8383.670\end{array}$ & $\begin{array}{l}12031.60 \\
12022.42 \\
12017.26 \\
11977.66 \\
11924.67\end{array}$ & $\begin{array}{l}200 \\
60 \\
20 \mathrm{cw} \\
40 \\
40\end{array}$ & & $\begin{array}{l}19895_{2_{1 / 2}}-31927^{0} o_{2_{1 / 2}} \\
53945_{2_{1 / 2}}-41922^{o} o_{2_{1 / 2}} \\
33480^{o} o_{1 / 2}-45497_{0_{1 / 2}}^{o_{1 / 2}} \\
314144_{3_{1 / 2}}^{o}-43391_{3_{1 / 2}}^{o_{1 / 2}} \\
33343_{1_{1 / 2}}^{o}-45267_{1_{1 / 2}}\end{array}$ \\
\hline $\begin{array}{l}8404.004 \\
8415.550 \\
8422.434 \\
8462.808 \\
8477.918\end{array}$ & $\begin{array}{l}11895.82 \\
11879.50 \\
11869.79 \\
11813.16 \\
11792.10\end{array}$ & $\begin{array}{c}7 \\
50 \mathrm{cw} \\
5 \\
10 \mathrm{c} \\
2\end{array}$ & & 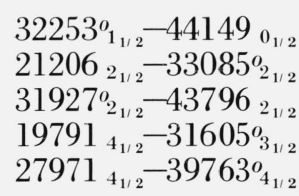 \\
\hline $\begin{array}{l}8481.348 \\
8484.000 \\
8497.126 \\
8514.700 \\
8522.539\end{array}$ & $\begin{array}{l}11787.34 \\
11783.65 \\
11765.45 \\
11741.16 \\
11730.37\end{array}$ & $\begin{array}{l}50 c \\
40 \\
5 h \\
30 \\
20\end{array}$ & & $\begin{array}{l}33480^{o} o_{0_{1 / 2}}-45267_{1_{1 / 2}} \\
20231_{1_{1 / 2}}-32014^{o_{2_{1 / 2}}} \\
33343_{1_{1 / 2}}^{o}-45108_{1_{1 / 2}} \\
31114^{o}{ }_{1_{1 / 2}}-42855_{4_{1 / 2}} \\
20523_{2_{1 / 2}}-32253_{1_{1 / 2}}^{o}\end{array}$ \\
\hline $\begin{array}{l}8531.062 \\
8537.653 \\
8543.616 \\
8595.933 \\
8604.090\end{array}$ & $\begin{array}{l}11718.65 \\
11709.60 \\
11701.43 \\
11630.21 \\
11619.18\end{array}$ & $\begin{array}{r}100 \\
30 \\
100 \\
10 \\
3\end{array}$ & & $\begin{array}{l}33085_{2_{1 / 2}}^{o}-448044_{2_{1 / 2}} \\
198955_{2_{1 / 2}}-31605_{3_{1 / 2}}^{o} \\
19903_{3_{1 / 2}}-31605_{3_{1 / 2}}^{\circ} \\
203843_{3_{1 / 2}}-320144_{2_{1 / 2}}^{o_{1 / 2}} \\
38319_{1_{1 / 2}}^{\circ}-49938_{2_{1 / 2}}\end{array}$ \\
\hline $\begin{array}{l}8639.395 \\
8664.128 \\
8673.629 \\
8707.215 \\
8719.950\end{array}$ & $\begin{array}{l}11571.70 \\
11538.67 \\
11526.03 \\
11481.57 \\
11464.80\end{array}$ & $\begin{array}{c}4 \\
6 \\
8 \\
100 \mathrm{cw} \\
30\end{array}$ & & $\begin{array}{l}20686_{2_{1 / 2}}-32258^{o} o_{1_{1 / 2}} \\
32258^{o}{ }_{1_{1 / 2}}-43796_{2_{1 / 2}} \\
161333_{4_{1 / 2}}-27660^{o}{ }_{3_{1 / 2}} \\
32620^{o} 3_{3_{1 / 2}}-44102_{3_{1 / 2}} \\
31927^{o_{2_{1 / 2}}}-43391_{3_{1 / 2}}\end{array}$ \\
\hline
\end{tabular}


TABLE 3. Classified lines of TC I

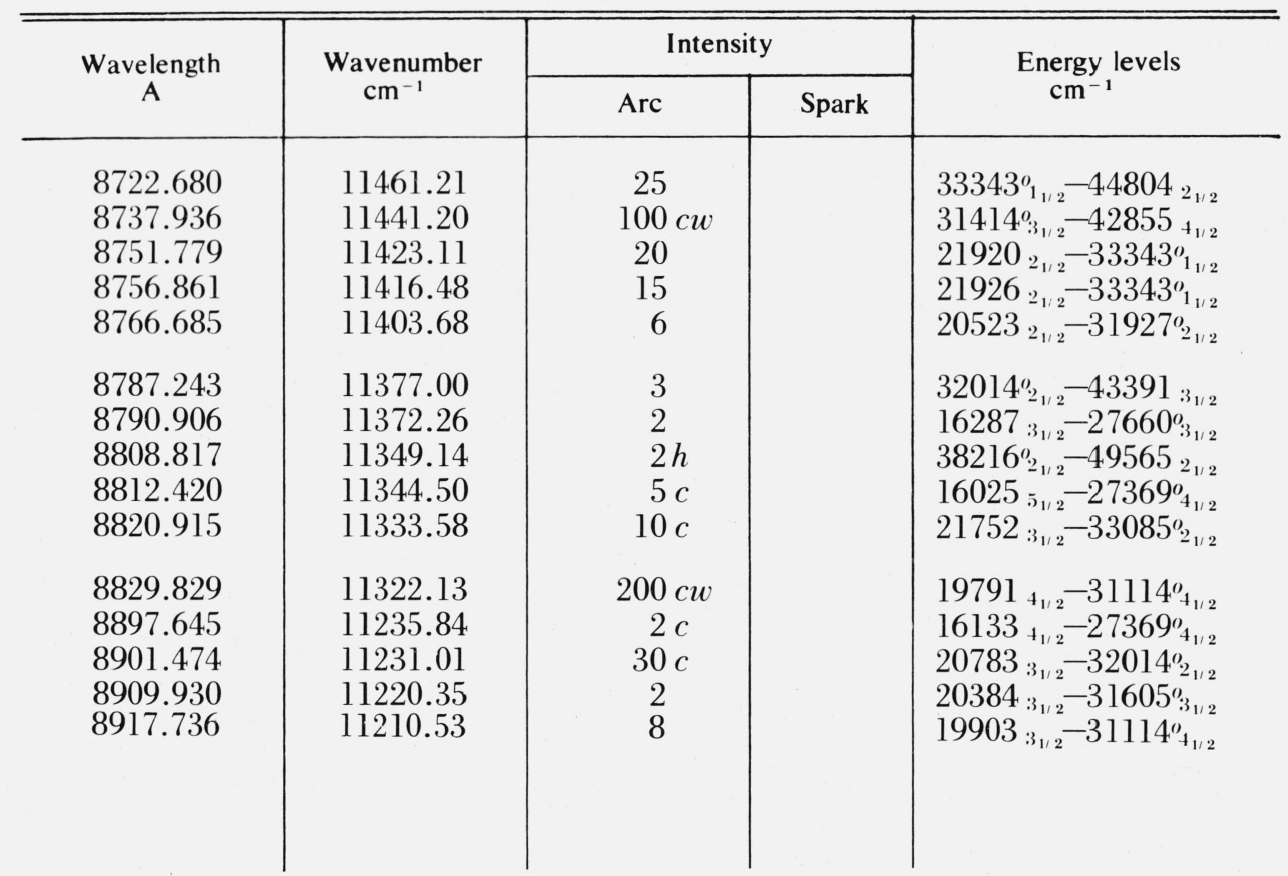

\section{References}

[1] Meggers, W. F., and Scribner, B. F., J. Res. NBS 45, 476 (1950).

[2] Meggers, W. F., J. Res. NBS 47, 7 (1951) RP2221.

[3] Bozman, W. R., J. Opt. Soc. Am. 44, 828(A) (1954).

[4] Bozman, W. R., J. Opt. Soc. Am. 46, 383(A) (1956).

[5] Bozman, W. R., Meggers, W. F., and Corliss, C. H., J. Res. NBS $71 \mathrm{~A}$ (Phys. and Chem.) No. 6, 547 (1967).
[6] Corliss, C. H., Bozman, W. R., and Westfall, F. O., J. Opt. Soc. Am. 43, 398 (1953).

[7] Moore, C. E., Atomic Energy Levels, Vol. III, NBS Circ. 467, p. 18 (Washington, U.S. Gov't Printing Office, 1958).

[8] Tech, J. L., NBS Publication in preparation.

(Paper 72A6-521) 